\title{
ON NEW RESULTS IN THE THEORY OF MINIMAL SURFACES
}

\author{
JOHANNES C. C. NITSCHE ${ }^{1}$ \\ Chapter I. Introduction
}

It was with some hesitation that I decided to talk about the theory of minimal surfaces. Although, over the centuries, minimal surfaces and the problems connected with them have attracted the never-ending interest of mathematicians, owing to their classical character and the fact that the theory, without doubt, reached its culmination in the 1930 's, ${ }^{2}$ marked by the spectacular and pioneering achievements of L. Tonelli, R. Garnier, T. Radó, J. Douglas, R. Courant, C. B. Morrey, E. M. McShane, M. Shiffman, M. Morse, T. Tompkins and others, one might expect to find a closed chapter. This is not the state of affairs, however.

New and important results have been forthcoming ever sinceso the results of M. Shiffman [3] on doubly-connected minimal surfaces, the studies of L. Bers [1], [2], [3] about Abelian minimal surfaces and about boundary-value problems for the minimal surface equation and isolated singularities of its solutions, the theorems of $\mathrm{H}$. Lewy [5], [6] on the boundary behavior of minimal surfaces, the papers of E. J. Mickle [2], E. Hopf [1], [2], E. Heinz [1], K. Jörgens [1], R. Osserman [1], [3], [4] on Bernstein's theorem and its generalizations, of $\mathrm{Y} . \mathrm{W}$. Chen [1], [2], [3] on new versions of Plateau's problem and on branch points, the ideas of R. Finn [1], $[2],[3],[6],[7]$ concerning the minimal surface equation and its generalizations, the publications of J. Serrin [2], H. Jenkins [1], [2], and $H$. Jenkins and J. Serrin [1] on a priori estimates for the minimal surface equation and more general variational problems, the work of R. Osserman [5]-[8] on complete minimal surfaces, studies of the relations between minimal surfaces in isothermic representation and function theory by M. Tsuji [1], M. O. Reade [1], E. F. Beckenbach and G. A. Hutchison [1], and many others, the manifold investiga-

An address delivered before the Chicago meeting of the Society on April 25, 1964, by invitation of the Committee to Select Hour Speakers for Western Sectional Meetings; received by the editor, August 28, 1964.

1 This work was supported by Contract Nonr 710 (54) between the University of Minnesota and the Office of Naval Research.

2 It might be of interest to note the following comment of two geometers (see W. Blaschke and H. Reichardt [1, p. 118]): "If one brings into comparison the first and the last of these works (i.e. the surveys by E. Beltrami [1] and T. Rad6 [6]) one can confront the stormy youth of a geometric question with its tired old age." 
tions of R. Courant (partly laid down in his book [3]) and his school, for instance, the solution of Schwarz's free boundary-value problem by I. F. Ritter [1], the proof of the bridge theorem by M. Kruskal [1], the study of unstable minimal surfaces by I. Marx [1], and a result of A. Solomon [1] on a special free boundary-value problem, the examples of P. Lévy [1], [2] of nonuniqueness in the Plateau problem, the extensive work of A. G. Sigalov (see, for instance, [1]), the remark of S. Sasaki [1] concerning branch points, new discussions of the isoperimetric inequality by A. Huber [1], [2], W. T. Reid [1], C. C. Hsiung [1], and, in a considerably more general setting, by S. $Z$. Sefel' $^{\prime}[1]$, detailed investigations of special minimal surfaces by $H$. Graf and $H$. Thomas [1], W. Wunderlich [1], [2], H. Jonas [1], M. Fréchet [1], R. Garnier [1], [2] and others-to recite only a few; not to mention the scores of papers on minimal surfaces in higherdimensional spaces and in Riemannian spaces, in affine, or projective, or complex geometry by D. V. Beklemišev, J. W. Lawson, A. T. Lonseth, K. Leichtweiss, Ü. Lumiste, C. B. Morrey, M. Pinl, K. Strubecker, and many others.

So it might be worthwhile, after all, to describe a few (and a selection of few it must remain) of the recent activities, as well as some of the difficulties, in the field of minimal surfaces. Some of these activities are concerned with a global or quantitative treatment of problems which had been dealt with before locally or in a qualitative manner, or with the study of old examples in the light of new questions, and thus at times appear (but only appear) to be a bit out-ofdate, at other times-as it seems almost unavoidable in a field so branched out as ours-lack complete novelty. Several of the results have not or not yet appeared elsewhere. It might be of interest also to have a small collection of problems, all classical in character, which have either defied so far attempts at solution or not yet been treated. Such a listing is given in Chapter VII.

Of course, the questions raised by the investigations over the years and the methods invented to deal with them in an adequate manner necessarily have led to considerable generalizations of the original concepts. This is particularly evident in the recent work of $E$. R. Reifenberg, as well as that of L. C. Young, E. de Giorgi, W. H. Fleming, H. Federer and others, which to a certain degree was motivated by the desire to obtain decent (at least locally decent) surfaces as solutions of Plateau's problem; surfaces which are not marred by the presence of branch points whose occurrence in the classical solutions - with a few notable exceptions even in seemingly reasonable cases-has never been proved to be avoidable. (To be sure, it has 
never been proved to be unavoidable either.) However, aside from the fact that these investigations pertain mainly to one aspect of the theory, namely, to the determination and study of varieties of smallest area with prescribed boundary, the methods involved are beyond the scope of this report. I shall restrict myself to the discussion of real ${ }^{3}$ two-dimensional parametric surfaces in ordinary 3-space, to a large part to such surfaces in a nonparametric (single-valued) representation $z=z(x, y)$, partly merely relating results, partly-if feasible in limited space, or if the known proofs are longer or more restrictivesketching proofs. Generalizations to two-dimensional surfaces and to $(n-1)$-dimensional hypersurfaces in Euclidean $n$-space are often possible and have been carried out by E. F. Beckenbach, S. S. Chern, J. Moser, R. Osserman and others. Occasionally they pose formidable problems, as, for instance, in the case of Bernstein's theorem. Only a few months ago the latter was proved for the case $n=4$ by $\mathrm{E}$. de Giorgi, employing concepts developed by R. Caccioppoli, W. H. Fleming, H. Federer, and himself, and a new regularity theorem due to D. Triscari.

The bibliography contains only papers relevant to the present report. For further references consult, for instance, R. Courant [3], J. Douglas [4], [6], R. V. Lilienthal [1], C. B. Morrey [3], T. Radó [6], M. Shiffman [2]. For the computations of the numerical estimates in the paragraphs II.9 and II.12, I am grateful to M. Stein and M. Engeli. The few historical footnotes are my own translation.

\section{Chapter II. The Minimal Surface Equation}

1. The minimal surface equation as a representative of nonlinear elliptic equations.

1.1. For a minimal surface, given in nonparametric representation $z=z(x, y)$, the function $z(x, y)$ is a solution of the minimal surface equation

$$
L[z] \equiv\left(1+q^{2}\right) r-2 p q s+\left(1+p^{2}\right) t=0 .
$$

It is a well-known fact, documented by striking examples, that the solutions of this equation behave quite differently from the solutions of linear elliptic differential equations. The reason lies in the severe nonlinearity of the minimal surface equation. This nonlinearity is so

3S. Lie [1, p. 332] makes the remark: "Following Riemann and Weierstrass one nowadays frequently investigates a real minimal surface by relating its real points to the real points of an $(x+i y)$-plane in the well-known manner. However beautiful, simple, and fruitful this approach may be, to me it seems a deficiency of it to take into consideration only the real points of real minimal surfaces." 
bad that it defies most of the general theories for elliptic equations which have been put forth in recent years. In fact, while it is the general aim of these investigations to prove the existence theorems, uniqueness theorems, regularity theorems, etc., characteristic for linear and mildly nonlinear equations, the minimal surface equation is a prototype of quite another class of differential equations for which new and different problems can, and have to, be considered.

1.2. Directing one's attention to the study of special properties of the solutions of certain classes of quasi-linear elliptic differential equations one can actually define measures of nonlinearity, conditions which, when fulfilled, guarantee the properties in question. This has been done by a number of authors in various ways and in regard to different properties of interest-solvability of Dirichlet's problem, possibility of isolated singularities, existence of nonlinear entire solutions, validity of certain a priori estimates, etc.; see, for instance, S. Bernstein [2], L. Bers [4], [5], R. Finn [2]-[7], H. Jenkins [1], [2], [3], H. Jenkins and J. Serrin [1], J. Leray [1], J. C. C. Nitsche [19], J. and J. Nitsche [1], [2], J. Serrin [1], [3]. Of course, in most cases these measures only lead to sufficient conditions.

The first such discussion was carried out by S. Bernstein in 1912 (see [2, especially pp. 455-469]) for quasi-linear elliptic differential equations

$$
L[z] \equiv a(x, y ; p, q) r+2 b(x, y ; p, q) s+c(x, y ; p, q) t=0,
$$

where $a c-b^{2}>0, a>0$, and, in greater generality, by J. Leray (see [1, especially pp. 281-283]). Let us say that a function $F(\xi, \eta)$ has a definite growth for $\xi$ and $\eta$ tending to infinity, and that this growth is of order $\alpha$ (in signs, $\left.F(\xi, \eta) \sim\left(\xi^{2}+\eta^{2}\right)^{\alpha / 2}\right)$, if there are two constants $m, \rho_{0}\left(1 \leqq m<\infty, \rho_{0}>0\right)$ and an exponent $\alpha$, such that the inequalities

$$
\frac{1}{m}\left(\xi^{2}+\eta^{2}\right)^{\alpha / 2} \leqq F(\xi, \eta) \leqq m\left(\xi^{2}+\eta^{2}\right)^{\alpha / 2}
$$

hold for $\xi^{2}+\eta^{2} \geqq \rho_{0}^{2}$. Under certain regularity conditions, and assuming that the two forms

$$
\begin{aligned}
& F_{1}=F_{1}(x, y ; p, q)=a p^{2}+2 b p q+c q^{2}, \\
& F_{2}=F_{2}(x, y ; p, q)=(a+c)\left(p^{2}+q^{2}\right)
\end{aligned}
$$

have, uniformly for all $(x, y)$ in the domain under consideration, a definite growth as $p$ and $q$ tend to infinity, of orders $\mu_{1}$ and $\mu_{2}$, respectively, S. Bernstein calls the difference $\mu=\mu_{2}-\mu_{1}$ the mode ("genre") of the differential equation (2). From the relation $F_{2}-F_{1}=a q^{2}-2 b p q$ 
$+c p^{2}$ it follows that $F_{2}>F_{1}$ for $p^{2}+q^{2} \neq 0$ and that $\mu_{2} \geqq \mu_{1}$, i.e., $\mu \geqq 0$. For the minimal surface equation we obtain $F_{1}=p^{2}+q^{2}$, $F_{2}=\left(p^{2}+q^{2}\right)\left(2+p^{2}+q^{2}\right)$, so that its mode is two. Linear and uniformly elliptic differential equations are of mode zero.

The investigations of $\mathrm{S}$. Bernstein concerning the solvability of Dirichlet's problem reveal that differential equations, whose mode does not exceed the value one, behave similarly to linear differential equations, while differential equations of mode exceeding one have different features. A particularly interesting class is that of the equations of mode two, the minimal surface equation being a representative of this class.

1.3. In 1954 and the following years R. Finn [3]-[7] introduced and investigated a class of quasi-linear differential equations which he, on account of their close relationship to the minimal surface equation, called equations of minimal surface type: An equation (2), under the normalization $a c-b^{2}=1$ and varying further conditions, is called of minimal surface type, if there is a constant $M(1 \leqq M<\infty)$ such that the inequality

$$
F_{3}=F_{3}(x, y ; p, q)=a\left(1+p^{2}\right)+2 b p q+c\left(1+q^{2}\right) \leqq 2 M W
$$

holds for all $(x, y)$ in the domain under consideration and for all $(p, q)$. Here, $W=\left(1+p^{2}+q^{2}\right)^{1 / 2}$. Note that $F_{2}=\left(p^{2}+q^{2}\right)\left(F_{3}-F_{1}\right)$.

Condition (4) has an interesting geometrical interpretation. It never seems to have been observed, however, and will be proved in J. C. C. Nitsche [19] that every equation of minimal surface type is an equation of mode two. The converse is not true, i.e., differential equations of mode two constitute a larger class than differential equations of minimal surface type. This may be illustrated by the differential equation (2) with coefficients

$$
\begin{aligned}
& a=\frac{1}{d}\left[1+W^{2(\alpha-1)}\left(-q+\beta \frac{p}{W}\right)^{2}\right], \\
& b=\frac{1}{d} W^{2(\alpha-1)}\left(-q+\beta \frac{p}{W}\right)\left(p+\beta \frac{q}{W}\right), \\
& c=\frac{1}{d}\left[1+W^{2(\alpha-1)}\left(p+\beta \frac{q}{W}\right)^{2}\right],
\end{aligned}
$$

where $d=\left[1+\omega^{2} W^{2(\alpha-1)}\left(1+\beta^{2} W^{-2}\right)\right]^{1 / 2}, \omega^{2}=p^{2}+q^{2}$, so that $a c-b^{2}=1$, and $\alpha>1$. Here we find $F_{1} \sim \omega^{\alpha}, F_{2} \sim \omega^{\alpha+2}, F_{3} \sim \omega^{\alpha}$.

1.4. A minimal surface $z=z(x, y)$ is solution, in a nonparametric representation, of the variational problem 


$$
\delta \iint\left[X^{2}+Y^{2}+Z^{2}\right]^{1 / 2} d u d v=0 .
$$

Here $X(u, v), Y(u, v)$, and $Z(u, v)$ denote the components of the vector product $\mathfrak{x}_{u} \times \mathfrak{x}_{v}$, where $\mathfrak{x}(u, v)$ is the vector of the surface in parametric representation. Let us consider a regular variational problem where the integrand is a positive and positive homogeneous $C^{2}$-function $F(X, Y, Z)$ of $X, Y$, and $Z$. An extremal in the form $z=z(x, y)$ of such a problem is extremal of a nonparametric problem

$$
\delta \iint F(p, q) d x d y=0 \quad(F(p, q)>0),
$$

whose Euler equation is of form (2). This connection has been investigated by A. Haar [3], T. Radó, and E. J. Mickle [1], and recently again by $H$. Jenkins [1], [2]. There are variational problems associated with (5) whose extremals are in the same relation to the extremals of (5) as adjoint minimal surfaces to each other. $H$. Jenkins proved the interesting fact that the Euler equation, derived from a problem whose indicatrix (i.e., the closed surface in $(X, Y, Z)$ space, defined by $F(X, Y, Z)=1)$ has everywhere positive Gaussian curvature, is of minimal surface type (more precisely, of class $\epsilon_{3}$; see R. Finn [3, p. 401]). Such equations, the solutions of which again exhibit the features of our paradigmatic minimal surface equation, have consequently been investigated by $\mathrm{H}$. Jenkins [2] and $\mathrm{H}$. Jenkins and J. Serrin [1].

1.5. In $1902 \mathrm{~S}$. A. Chaplygin [1] observed that the minimal surface equation may be interpreted as the potential equation of a steady gas flow whose velocity $\omega=\left(Z_{x}^{2}+Z_{y}^{2}\right)^{1 / 2}$ and density $\rho$ are connected by the relationship $\rho^{2}\left(1+\omega^{2}\right)=1$. Although this equation implies the physically impossible pressure-density relation $p=a+b / \rho$, it turns out that "Chaplygin flows" are good approximations to sufficiently slow flows of an actual gas. L. Bers [1] and Y. W. Chen [2], [3] have formulated and investigated a number of exterior boundaryvalue problems for the minimal surface equation, representing a steady Chaplygin flow past a given profile, which were suggested by this gas-dynamical interpretation, applying fixed-point theorems to resulting integral equations, or solving new parametric problems, respectively.

1.6. All these investigations, many of which easily carry over to the $n$-dimensional case, center around the minimal surface equation as the most prominent representative of a new class of equations with special features, and the discussion in the following paragraphs 
may well be considered as a model and a program for the investigation of a broad category of quasi-linear elliptic differential equations in two as well as in $n$ dimensions.

\section{Nonsolvability of Dirichlet's problem.}

2.1. Given a Jordan domain $P$ in the $(x, y)$-plane and a continuous function $f(x, y)$, defined on its boundary $\partial P$, characterizing a simple closed curve $\Gamma$ in $(x, y, z)$-space. The nonparametric Plateau problem, i.e., the Dirichlet problem for the minimal surface equation, calls for a solution $z(x, y) \in C^{2}(P) \cap C^{0}(\bar{P})$ of this equation in $P$, whose values on $\partial P$ coincide with those of the function $f$.

In $1832 \mathrm{~S}$. D. Poisson [1] announced that he had obtained an existence proof for the case where the curve $\Gamma$ differs only slightly from a plane curve. He never published this proof, however. In 1909 such a proof was put forward in a classical paper by A. Korn [1]. Korn assumes the boundary $\partial P$ as well as the function $f$ to belong to class $C^{2, \lambda}$ and requires the $(2, \lambda)$-norm of $f$ to be sufficiently small. In later years the problem was dealt with in increasing generality (and increasing rigor ${ }^{4}$ ) in the work of S. Bernstein [1], [2], C. H. Müntz [1], A. Haar [2], and especially T. Radó [4], and it is now a wellestablished fact that a solution exists for arbitrary continuous boundary data, provided the domain $P$ is convex (not necessarily strictly convex).

The simplest proof (much simpler than the one using methods of partial differential equations) applies a lemma of T. Rado to the parametric solution of Plateau's problem (see T. Radó [4, pp. 795796], [5, p. 16], [6, p. 35]). If one takes for granted the solvability of Dirichlet's problem for small circles with continuous boundary data and employs the a priori estimates and compactness theorems of paragraphs II.12 and II.13, a modification of the method devised by O. Perron (see [1]; also R. Courant and D. Hilbert [1, pp. 306312]) can also be used. Barriers are easily constructed in all boundary points in which the boundary is "locally convex." (The boundary $\partial P$ is called locally convex in one of its points $p$, if there is an open circle $K$ with center $p$, such that the component of $P \cap K$ which contains $p$ as boundary point is convex.)

2.2. For general domains the situation is more complex. This was first pointed out by S. Bernstein (see [2, especially pp. 455-469]) and discussed in greater generality by J. Leray [1]. Bernstein outlined a proof that for equations of mode two there are nonconvex domains

4 Some controversial arguments concerning this question can be found in S. Bernstein [3], T. Rad6 [3], C. H. Müntz [2]. 


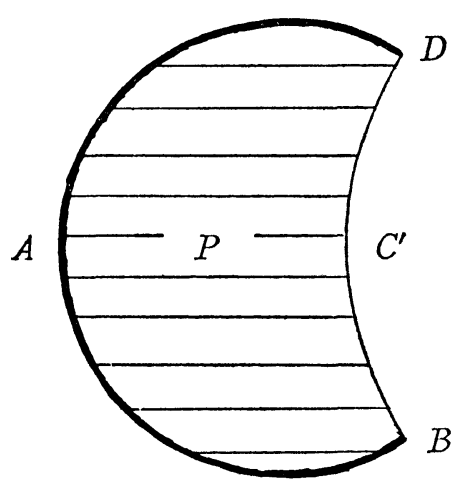

Figure 1

for which Dirichlet's problem, in general, is not solvable. The domains $P$ he considered are bounded in part by a concave analytic arc $B C^{\prime} D$, as in Figure 1, and his proof is based on the construction of an auxiliary function $z=\phi(x, y)$, a solution of class $C^{2}(P) \cap C^{0}(\bar{P})$, whose gradient tends to infinity upon approach of any point of the arc $B C^{\prime} D$.

If now $z(x, y)$ is a solution of the minimal surface equation in $P$, whose values along the arc $B A D$ (see Figure 1) do not exceed those of the auxiliary function $\phi$, an extension of the maximum principle shows that the inequality $z \leqq \phi$ must hold in all of $\bar{P}$. Thus Dirichlet's problem cannot be solvable if the prescribed boundary data, while remaining smaller than $\phi$ along the arc $B A D$, at some point of the arc $B C^{\prime} D$ exceed the value of the function $\phi$.

2.3. The auxiliary function $\phi(x, y)$ is obtained as solution of a Cauchy problem in sufficiently small domains. It should be pointed out that in the case of the minimal surface equation this Cauchy problem is nothing but Björling's problem: Choose a concave curve $\mathcal{e}$ in the $(x, y)$-plane (concave as viewed from a point with large abscissa). Determine the minimal surface $S$, given in parametric representation $\mathfrak{x}=\mathfrak{x}(u, v)$, which contains $\mathfrak{C}$ and whose normal vector, in the points of $\mathfrak{C}$, lies in the $(x, y)$-plane. On $S$ the curve $\mathcal{C}$ must be a curvature line, in fact, a geodesic. The curves of intersection of $S$ with the planes $y=$ const are vertical, i.e., parallel to the $z$-direction, in the points of $\mathcal{C}$ and convex in a neighborhood of $\mathcal{C}$ (convex as viewed from a point with large abscissa). It will be possible, therefore, at least locally, to project the part of $S$ below the $(x, y)$-plane in a single-valued manner upon the $(x, y)$-plane, giving rise to a non- 
parametric representation $z=z(x, y)$ of $S$. The function $z(x, y)$ has all the properties of the auxiliary function $\phi(x, y)$ of Bernstein.

The determination of a minimal surface, containing a given plane curve $\mathfrak{C}$ as geodesic, is a standard exercise and has been carried out explicitly for many special cases in the work of L. Henneberg [1], [2], A. Herzog [1], H. W. Richmond (see [1, especially pp. 238-239]), H. A. Schwarz (see [3, especially p. 199], [2, especially p. 182]), H. Tallquist [1] and others. If $\mathfrak{C}$ is a circle, we obtain a catenoid; if $\mathfrak{C}$ is a cycloid, we obtain E. Catalan's surface (see [1, pp. 161-164]); but also ellipses, parabolas, Neil's parabolas, asteroids, and other curves have been discussed.

Let us give here three explicit examples of minimal surfaces $z=z(x, y)$ having the properties of the auxiliary function $\phi$ over special domains. Each leads, using Bernstein's idea, to quantitative statements. The first has recently been used in an interesting way by R. Finn [7] for such purposes.

(i) $z=\phi_{1}(x, y ; a, b)=a \log \left[\left(b+\left(b^{2}-a^{2}\right)^{1 / 2}\right) /\left(r+\left(r^{2}-a^{2}\right)^{1 / 2}\right)\right]$, where $0<a<b$ and $r=\left(x^{2}+y^{2}\right)^{1 / 2}$ (catenoid).

(ii) The minimal surface $z=\phi_{2}(x, y ; a)$, defined parametrically by the equations

$$
\begin{aligned}
& x=a\left[u+u v^{2}-\frac{1}{3} u^{3}\right], \\
& y=a\left[-v-u^{2} v+\frac{1}{3} v^{3}\right], \\
& z=a\left[u^{2}-v^{2}\right]
\end{aligned}
$$

(Enneper's surface, see [1]).

$$
z=\phi_{3}(x, y ; a)=\frac{2^{1 / 2}}{a} \sin ^{-1}\left[\frac{1}{2} \cosh (a x)-\frac{1}{2} \cosh (a y)\right],
$$

obtained by a simple transformation from the minimal surface $\sin z=\sinh x \sinh y$, which was already known to H. F. Scherk [1].

All these functions vanish on the heavily drawn part $B A D$ of the boundary, while their gradient tends to infinity upon approach of any point of the thinly drawn part $B C^{\prime} D$ of the boundary; see Figures $2 a, 2 b, 2 c$, which show the domains for the functions $\phi_{i}$.

Added in proof. As a matter of fact, a simple argument in combination with the result of R. Finn [7, Theorem 4] yields the following interesting conclusion: For every nonconvex Jordan curve there exist continuous boundary data for which Dirichlet's problem is not solvable. 


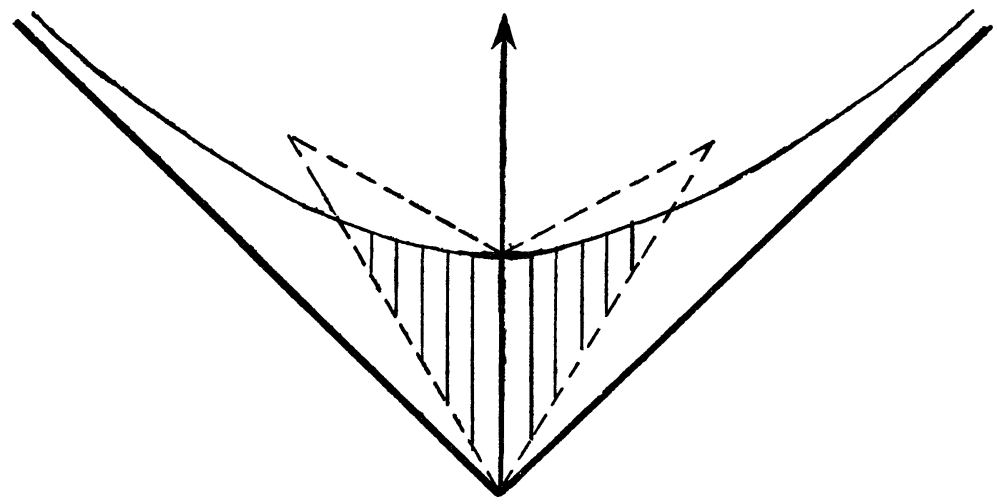

FIGURE 2a

Domain for the function $\phi_{3}(x, y ; a)$

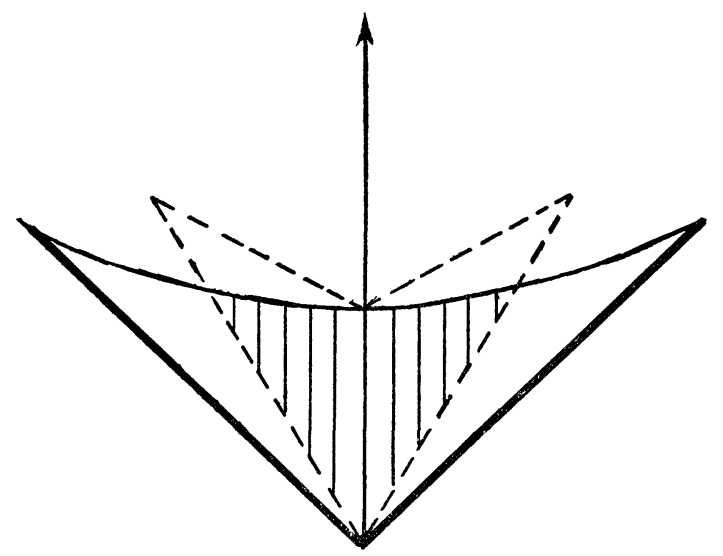

FIGURE $2 b$

Domain for the function $\phi_{2}(x, y ; a)$

2.4. A particularly clear-cut example for the nonsolvability of Dirichlet's problem, often cited in the literature, was given by $\mathrm{T}$. Radó (see [5, pp. 18-20], [6, p. 37]) as an application of his uniqueness theorem [5, p. 10], [6, pp. 35-37] to the classical minimal surface through four sides of a regular tetrahedron, determined by $\mathrm{H}$. A. Schwarz [1] and B. Riemann [1, pp. 326-329]. Here the domain $P$ is the nonconvex quadrilateral $A B C^{\prime} D$ (see Figure 3), and the boundary values $f$ are zero on the sides $A B$ and $A D$ and increase linearly from zero to the value $h_{0}=(2 / 3)^{1 / 2}$ along the sides $B C^{\prime}$ and $D C^{\prime}$. Let us consider, more generally, for the quadrilateral $A B C^{\prime} D$, the Dirichlet problem $\mathfrak{B}_{h}$, where the boundary values are again zero on the sides 


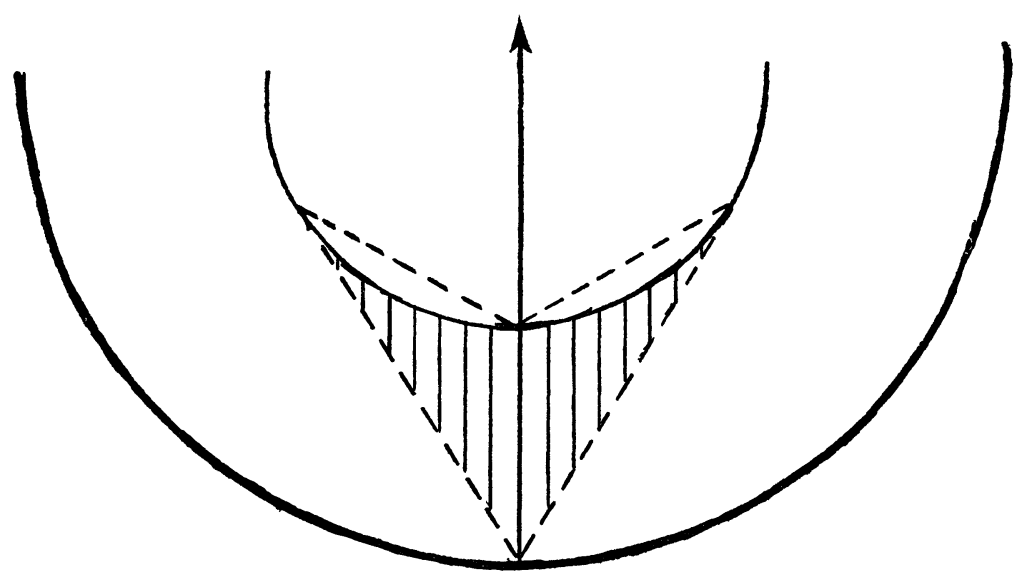

FIGURE 2c

Domain for the function $\phi_{1}(x, y ; a, b)$

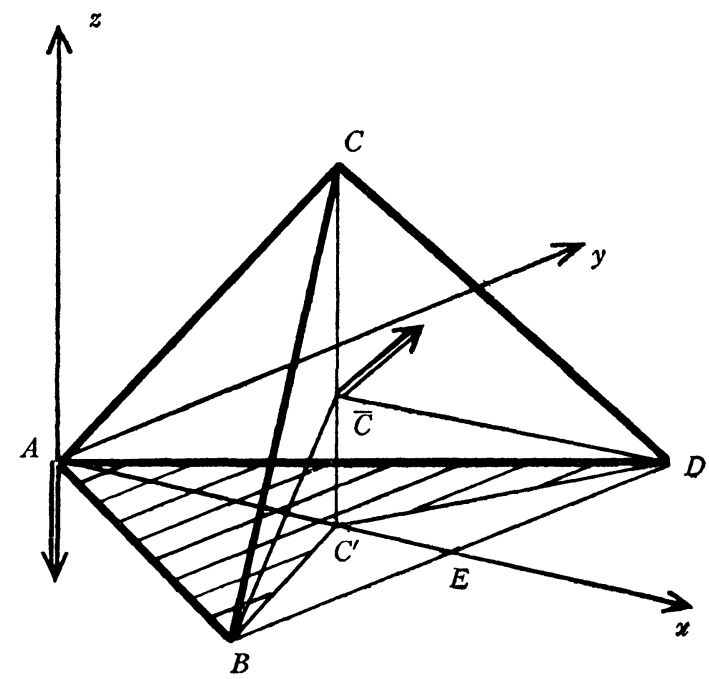

FIGURE 3

$$
\begin{array}{ll}
A=(0,0,0) & B=\left(\frac{1}{2}(3)^{1 / 2},-\frac{1}{2}, 0\right) \\
C^{\prime}=\left(\frac{1}{3}(3)^{1 / 2}, 0,0\right) & D=\left(\frac{1}{2}(3)^{1 / 2}, \frac{1}{2}, 0\right) \\
C=\left(\frac{1}{3}(3)^{1 / 2}, 0,\left(\frac{2}{3}\right)^{1 / 2}\right) & C=\left(\frac{1}{3}(3)^{1 / 2}, 0, h\right)
\end{array}
$$

$A B$ and $A D$ and increase linearly from zero to the value $h \geqq 0$ along the sides $B C^{\prime}$ and $D C^{\prime}$, i.e., we are asking for a minimal surface $z=z(x, y)$ through the polygon $\Gamma$ with corners $A, B, \bar{C}$, and $D$ (see Figure 3). Since the minimal surface of Schwarz and Riemann is uniquely determined and passes through the center of the tetrahedron 
$A B C D$, i.e., has no single-valued projection onto the $(x, y)$-plane, it is clear that problem $\mathfrak{B}_{h}$ has no solution for $h=h_{0}=0.8165 \cdots$. If one applies the idea of S. Bernstein, comparing the solution $z(x, y)$ with any one of the auxiliary functions $\phi$ mentioned earlier (under proper choice of the parameters $a, b$ ) in the shaded regions indicated in Figures $2 \mathrm{a}, 2 \mathrm{~b}, 2 \mathrm{c}$ one finds that problem $\mathfrak{P}_{h}$ is not solvable, respectively, for

$$
\begin{array}{rlrl}
h>h_{1} & =\frac{1}{3^{1 / 2}} \log \left(2+3^{1 / 2}\right)=0.760 \ldots & & \text { (using } \left.\phi_{1}\right), \\
h>h_{2} & =\frac{1}{2}(3)^{1 / 2}=0.866 \cdots & & \text { (using } \left.\phi_{2}\right), \\
h>h_{3} & =\pi\left[(24)^{1 / 2} \log \left(1+2^{1 / 2}\right)\right]^{-1} & \\
& =0.727 \ldots & \left.\quad \text { (using } \phi_{3}\right) .
\end{array}
$$

The first inequality was recently obtained by R. Finn [7].

In view of the result of A. Korn-disregarding his smoothness assumptions-one might be inclined to think that problem $\mathfrak{P}_{h}$ will be solvable at least for sufficiently small values of $h$ (problem $\mathfrak{P}_{0}$ has the trivial solution $z(x, y)=0)$; and, surprisingly, it has been an open question until now whether this is the case or not. I say "surprisingly," since the impossibility of a solution is intuitively clear: The unique minimal surface in question, bounded by the polygon $\Gamma$ and given in parametric representation, would have in the points $A$ and $\bar{C}$ normal vectors as indicated in Figure 3 . Obviously it would not lend itself to a nonparametric representation $z=z(x, y)$. In a forthcoming paper (see J. C. C. Nitsche [18]) it is proved that

problem $\mathfrak{P}_{h}$ has no solution for $h>0$.

The proof extends without difficulties to similar problems on any nonconvex quadrilateral.

2.5. If one attacks problem $\mathfrak{P}_{h}$ by the method of O. Perron (see II.2.1), one obtains a solution of the minimal surface equation in the interior of the quadrilateral $A B C^{\prime} D$. This solution assumes the boundary values in all boundary points in which the boundary is locally convex, i.e., in all boundary points with the exception of the vertex $C^{\prime}$ of the re-entrant angle. Exactly how the Perron-solution behaves in a neighborhood of the point $C^{\prime}$ apparently has never been investigated. The same remark applies to the general question how the Perron-solution behaves in a vicinity of a boundary point in which the boundary is not locally convex.

3. Dirichlet's problem with incomplete boundary data. Another of the surprising differences between the properties of harmonic func- 
tions and solutions of the minimal surface equation is contained in the famous theorem of L. Bers [2] to the effect that an isolated singularity of a (single-valued) solution of the minimal surface equation, interior to the domain of definition, ipso facto, must be removable. In particular, a solution of the minimal surface equation remains bounded in the neighborhood of such a singularity - a fact which may be interpreted as an extended maximum principle:

Let $z(x, y)$ be a solution of the minimal surface equation in the punctured disc $P_{0}=\left\{x, y ; 0<x^{2}+y^{2}<r^{2}\right\}$. Let $m=\lim \inf z(x, y)$ and $M=\lim \sup z(x, y)$ for any approach to the circle $x^{2}+y^{2}=r^{2}$. Then the inequalities $m \leqq z(x, y) \leqq M$ hold in all of $P_{0}$.

Other proofs of the extended maximum principle and of the theorem of $\mathrm{L}$. Bers, often valid for classes of quasi-linear elliptic equations, have been given by L. Bers [5], R. Finn [2], [7], H. Jenkins [3], J. C. C. Nitsche [6], [19], Johannes and Joachim Nitsche [2]. The extended maximum principle (also in the more general form given in the last reference) holds for all elliptic differential equations (2) whose mode is greater than one or, more generally, for which the improper integral

$$
\int^{\infty} \frac{d \omega}{\omega Q(\omega)}
$$

converges. Here

$$
Q(\omega)=\operatorname{Min}_{p^{2}+q^{2}=\omega^{2}}\left\{\operatorname{Inf}_{(x, \nu)}\left(\frac{F_{2}(x, y ; p, q)}{F_{1}(x, y ; p, q)}-1\right)\right\} .
$$

(J. C. C. Nitsche [19].)

While it is easy to construct solutions of (1) for which an isolated singularity on the boundary of the domain of definition is not removable, the extended maximum principle still holds. This was first proved by Johannes and Joachim Nitsche [2] and, in greater generality, recently by R. Finn [6, p. 342]. Based on this maximum principle the author had discussed in [4], some years ago, Dirichlet's problem for a bounded convex (not necessarily strictly convex) domain with boundary data which are continuous and bounded, but which are not defined in finitely many boundary points. For harmonic functions this problem makes no sense without the imposition of additional growth conditions. Thus the problem deals not with the question of the regularity or irregularity of a boundary point but rather with the question of the existence and uniqueness of the solution under incomplete boundary data.

It is now possible to generalize and solve Dirichlet's problem to the extent that the prescribed boundary values are continuous and bounded 
except on a compact set on $\partial P$ of vanishing linear Lebesgue measure (taken with respect to the arc length on $\partial P$ ).

Details are given in J. C. C. Nitsche [16].

4. Sets of vanishing linear Hausdorff measure. A point set $A$ on a convex curve of vanishing linear Lebesgue measure, considered as point set in the plane, has also vanishing linear Hausdorff measure. It is for this as well as for other reasons that exceptional sets of vanishing linear Hausdorff measure play a role in various investigations of interest for us. Among other things important for these investigations is the fact that a compact set $A$ of vanishing linear Hausdorf measure, contained in the closure $\bar{P}$ of a domain $P$, does not separate this domain. Considering that the point sets of vanishing linear Hausdorff measure generally are somewhat bigger than the point sets of vanishing logarithmic capacity (see O. Frostman $[1$, p. 86]; L. Carleson $[1$, p. $32]$ ), this statement contains a basic theorem of function theory. In the next lines a simple quantitative proof is given for this property in the following version:

Let $A$ be a compact set of vanishing linear Hausdorff measure, contained in the closure $\bar{P}$ of the circular annulus $P=\left\{x, y ; 0<r_{1}^{2}<x^{2}<y^{2}\right.$ $\left.<r_{2}^{2}<\infty\right\}$. It is possible to find a Jordan curve in $P$ of length arbitrarily close to $2 \pi r_{1}$, containing the origin $x=y=0$ in its interior, and avoiding the set $A$.

For the proof choose a number $\epsilon\left(0<\epsilon<\operatorname{Min}\left(2 r_{1},\left(r_{2}-r_{1}\right) / \pi\right)\right)$. The set $A$ can be covered by finitely many open circles, the sum of whose diameters is less than $\epsilon$. The union of these circles consists of a finite number of open components $O_{1}, O_{2}, \cdots, O_{m}$ (depending on $\epsilon$ ). Each component $O_{i}$ is bounded by circular arcs. The length of its boundary cannot exceed the total circumference of the circles participating in its formation and thus is certainly not larger than $\pi \epsilon<\operatorname{Min}\left(2 \pi r_{1}, r_{2}-r_{1}\right)$. From this fact it follows that the closure of no component $O_{i}$ can have points in common simultaneously with the inner and the outer boundaries of $\bar{P}$ and that there are points on the circle $x^{2}+y^{2}=r^{2}$ $\left(r_{1}<r<r_{2}\right)$ not covered by the $O_{i}$. Starting from such a point we now follow the circle $x^{2}+y^{2}=r^{2}$. If we meet a component $O_{i}$, say $O_{1}$, we follow its boundary in such a way that we, without leaving the annulus $P$, come back to the circle $x^{2}+y^{2}=r^{2}$ on "the other side" of $O_{1}$. In this fashion we continue. We might encounter the component $O_{1}$ repeatedly. The new detours, however, each time follow new parts of its boundary. The total detour on this journey once around the circle $x^{2}+y^{2}=r^{2}$ cannot amount to more than $\pi \epsilon$. The assertion follows if we choose $r$ sufficiently close to $r_{1}$. (A simpler proof, com- 
municated to the author by W. Littman, can be found in J. C. C. Nitsche [16].)

5. The general maximum principle.

5.1. The uniqueness proof for the general Dirichlet problem of II.3 is based on a general maximum principle. It contains as special cases the extended maximum principle of II.3 and maximum principles proved by Johannes and Joachim Nitsche [2], R. Finn [6], and J. Serrin (in an unpublished remark).

Let $P$ be a bounded domain in the $(x, y)$-plane and $A \subset \bar{P}$ a compact set of vanishing linear Hausdorff measure. Let $z_{1}(x, y)$ and $z_{2}(x, y)$ be two solutions of class $C^{2}$ of the minimal surface equation, defined in $P-A$. Assume that lim sup $\left[z_{2}(x, y)-z_{1}(x, y)\right] \leqq M$ (lim inf $\left.\left[z_{2}(x, y)-z_{1}(x, y)\right] \geqq m\right)$ for approach of any boundary point of $P$ not in $A$. Then the inequality $z_{2}(x, y)-z_{1}(x, y) \leqq M\left(z_{2}(x, y)-z_{1}(x, y)\right.$ $\geqq m$ ) holds in all points of $P-A$.

For the proof the reader is referred to J. C. C. Nitsche [16]. It should be pointed out that the exceptional set $A$ may well lie entirely on the boundary of $P$.

5.2. It is not clear whether the linear Hausdorff measure leads to optimal results. The question to find necessary and sufficient metric conditions on the exceptional set $A$ for the general maximum principle to hold, is still open.

From the results of II.7 it is easy to construct examples where the exceptional set is a straight line segment on the boundary or in the interior of the domain of definition (and thus has positive linear Hausdorff measure), for which the general maximum principle does not hold. In fact the simplest, although rather crude, example is given by Scherk's surface (6); see II.7.1.

R. Finn [7, pp. 7-8] has also given an example of a solution $z(x, y)$ of the minimal surface equation, defined in the neighborhood of a straight line segment, which tends to $+\infty$ for approach of any interior point of this segment. Comparing $z(x, y)$ with the function $\phi_{1}(x, y ; a, b)$ of II.2.3 he shows that $z(x, y)$ cannot tend to $+\infty$ for approach of all points of the segment. This is also a consequence of the results of IV.2 and V.4. As a matter of fact, the following quantitative statement, given here without proof, holds:

Let $P$ be a domain, contained in a circle of radius $r$, and $B$ a compact set in the interior of $P$. Let $z(x, y)$ be a solution of the minimal surface equation in $P-B$. If $\lim \sup z(x, y) \leqq m$ for approach of every boundary point of $P$ and $\lim \inf z(x, y) \geqq M$ for approach of every point of $B$, then the inequality $M-m \leqq 2 r / 3$ must hold. 
5.3. The general maximum principle plays a basic role for the considerations of the paragraphs II.6 to II.12. It also has many other special applications. For instance, in combination with a barrier argument the following statement can be proved:

Let $z(x, y)$ be a solution of the minimal surface equation in a Jordan domain $P$. Let $p$ be a boundary point in which the boundary $\partial P$ is locally convex (see II.2.1). Let $K$ be an open circle containing $p$, and assume that $\lim \sup z(x, y) \leqq M(\lim \inf z(x, y) \geqq m)$ for approach of any point of $K \cap \partial P-p$. Then $\lim \sup z(x, y) \leqq M(\lim \inf z(x, y) \geqq m)$ also for approach of $p$.

6. Removable singularities. The theorem of $L$. Bers about the removability of an isolated point singularity has already been mentioned in II.3. In the investigation of the removability of singularities of harmonic functions one does not restrict oneself to the consideration of isolated exceptional points. There are many results on the removability of singularities for certain classes of harmonic functions -bounded functions, Lipschitz-continuous functions, functions with bounded Dirichlet integral or bounded integral norm-on sufficiently meager sets wholly interior to the domain of definition. (See the surveys of M. Tsuji [2] and L. Carleson [1] which contain extensive bibliographies.) Recently J. Serrin [3] has investigated such questions for a class of nonlinear differential equations containing the Laplace equation as special case. Although the minimal surface equation eludes his assumptions and, in fact, exhibits quite different features, his method, in combination with an argument of R. Finn [2], extends to a certain degree to the minimal surface equation provided, however, among other things, that the exceptional set is contained in the interior of the domain of definition. On the basis of the solvability of the general Dirichlet problem, of the general maximum principle, and of the properties of sets of vanishing linear Hausdorff measure the following theorem on removable singularities holds (see J. C. C. Nitsche [16]):

Let $P$ be a bounded domain in the $(x, y)$-plane and $A \subset \bar{P}$ a compact set of vanishing linear Hausdorff measure. (Note that the exceptional set $A$ is permitted to extend to the boundary of $P$.) Let $z(x, y) \in C^{2}(P-A)$ be a solution of the minimal surface equation in $P-A$. The set $A$ is removable for the solution $z(x, y)$, i.e., the function $z(x, y)$ can be assigned such values in the points of the set $A$ that the extended function is a $C^{2}$ solution of the minimal surface equation in all of $P$. The extension is unique.

For the proof let $\left(x_{0}, y_{0}\right)$ be a point of $A \cap P$, and let $K_{1}$ and $K_{2}$ be two open circles about this point $\left(\bar{K}_{1} \subset K_{2}, \bar{K}_{2} \subset P\right)$. By II.4, we can 
find, in the annulus $\bar{K}_{2}-K_{1}$, a Jordan curve containing the point $\left(x_{0}, y_{0}\right)$ in its interior and avoiding the set $A$. Along this curve the solution is defined and hence bounded. By the general maximum principle the solution is bounded in all of $\bar{K}_{1}-A$. We now solve the general Dirichlet problem II.3 for the circle $K_{1}$ with the values of $z(x, y)$ prescribed on $K_{1}-A$. Its solution $Z(x, y)$ has continuous second derivatives in $K_{1}$. Applying again the general maximum principle, it is seen that $Z(x, y)$ must coincide with $z(x, y)$ in $\bar{K}_{1}-A$. In this way an extension of the function $z(x, y)$ as solution of the minimal surface equation to all of $P$ is obtained. It is easy to show that this extension must be unique.

7. Dirichlet's problem with partly infinite boundary data.

7.1. According to II.3 we can solve Dirichlet's problem for the special case in which the boundary values are piecewise smooth and partly equal to zero, partly equal to a nonzero constant. The solution is identical with the solution of the parametric Plateau problem whose boundary contour possesses vertical segments. A century ago many special minimal surfaces were considered whose boundaries contain infinite half-lines. An example is provided by the surface of $\mathrm{H}$. F. Scherk [1]:

$$
\begin{aligned}
z=\frac{a}{2}\{\log \cos & {\left[\frac{1}{a}\left(\frac{x}{\cos \alpha}-\frac{y}{\sin \alpha}\right)\right] } \\
- & \left.\log \cos \left[\frac{1}{a}\left(\frac{x}{\cos \alpha}+\frac{y}{\sin \alpha}\right)\right]\right\},
\end{aligned}
$$

where $0<\alpha<\pi / 2$ and $a$ is a positive parameter. This surface has been extensively studied in the literature. It is real only over the "black rhombi"

$$
\left|\frac{x}{\cos \alpha}-\frac{y}{\sin \alpha}-2 m a \pi\right|<\frac{a \pi}{2}, \quad\left|\frac{x}{\cos \alpha}+\frac{y}{\sin \alpha}-2 n a \pi\right|<\frac{a \pi}{2}
$$

$(m, n=0, \pm 1, \pm 2, \cdots)$ of an infinite skew checkerboard. For $\alpha=\pi / 4, a=2 b$, and after a rotation of the coordinate system by $45^{\circ}$, the well-known special surface

$$
z=b\{\log \cos (y / b)-\log \cos (x / b)\}
$$

is obtained. Let us consider the surface (6) only over the right triangle with vertices $0=(0,0), p_{1}=((\pi a / 2) \cos \alpha, 0)$, and $p_{2}=$ $(0,(\pi a / 2) \sin \alpha)$. We see that $z(x, y)=0$ in all points of the sides of this triangle with the exception of $p_{1}$ and $p_{2}$, while $z(x, y)$ tends to $+\infty$ upon approach of every interior point of the hypotenuse. 
Another example is given by the minimal surface

$$
z=\frac{1}{a} \sinh ^{-1}[\sinh (a x) / \sinh (a y)], \quad a>0
$$

(see example (iii) in II.2.3). This surface is defined over any rectangle $R=\{x, y ; 0<x<\pi / a, 0<y<b\}$, where $b>0$. Approaching the top and vertical sides of $R$, with the exception of the points $(0,0)$ and $(\pi / a, 0), z(x, y)$ tends to finite limits, while it tends to $+\infty$ approaching the segment $0<x<\pi / a$ of the $x$-axis. Actually, $z(x, y)$ is defined over the half-plane $y>0$ (as well as over the half-plane $y<0$ ) and tends to $+\infty$ for approach of the segments $2 n \pi / a<x<(2 n+1) \pi / a$ of the $x$-axis and to $-\infty$ for approach of the segments $(2 n-1) \pi / a$ $<x<2 n \pi / a$ of the $x$-axis.

7.2. Recently H. Jenkins and J. Serrin (in still unfinished work, see also [1]) and R. Finn [6] have directed their attention to generalizations of this situation. Let $P$ be a bounded convex (not necessarily strictly convex) domain in the $(x, y)$-plane. Let $\partial P_{1}$ and $\partial P_{2}$ be open, complementary subarcs of its boundary $\partial P$ with joint endpoints $q_{1}$ and $q_{2}$. On $\partial P_{1}$ a continuous bounded function $\phi$ is given: $|\phi| \leqq M$.

The problem calls for a solution $z(x, y) \in C^{2}(P) \cap C^{0}\left(P \cup \partial P_{1}\right)$ of the minimal surface equation, whose values on $\partial P_{1}$ coincide with those of the function $\phi$ and which tends to $+\infty$ upon approach of any point on $\partial P_{2}$.

Considering that R. Finn treats under special circumstances only certain aspects of the problem important for his purposes (for instance he does not discuss the question of uniqueness), and that the work of $\mathrm{H}$. Jenkins and J. Serrin is still incomplete, the following treatment gives in various points my own version of the proofs.

By a simple argument (see II.11) it is seen that our problem can be solvable only if the arc $\partial P_{2}$ is a straight segment. The solution is obtained as the limit for $n \rightarrow \infty$ of solutions of the general Dirichlet problem II.3 with boundary values $\phi$ on $\partial P_{1}$ and $n$ on $\partial P_{2}$ $(n=1,2, \cdots)$.

The uniqueness involves more delicate considerations. The simplest proof seems to proceed as follows. For concreteness let us assume that the domain $P$ lies in the half-plane $y>0$ and that $\partial P_{2}$ is the segment $-a<x<a$ of the $x$-axis (see Figure 4). First one proves two facts:

(i) Let $z_{1}(x, y)$ and $z_{2}(x, y)$ be two solutions of our problem. There is a number $N$, depending only on the domain $P$ and on the bound $M$ for the function $\phi$ on $\partial P_{1}$, such that $\left|z_{2}(x, y)-z_{1}(x, y)\right| \leqq N$ everywhere in $P \cup \partial P_{1}$.

(ii) Let $(x, y)$ be a point of $P$ whose distance $d=d(x, y)$ from $\partial P_{1}$ 


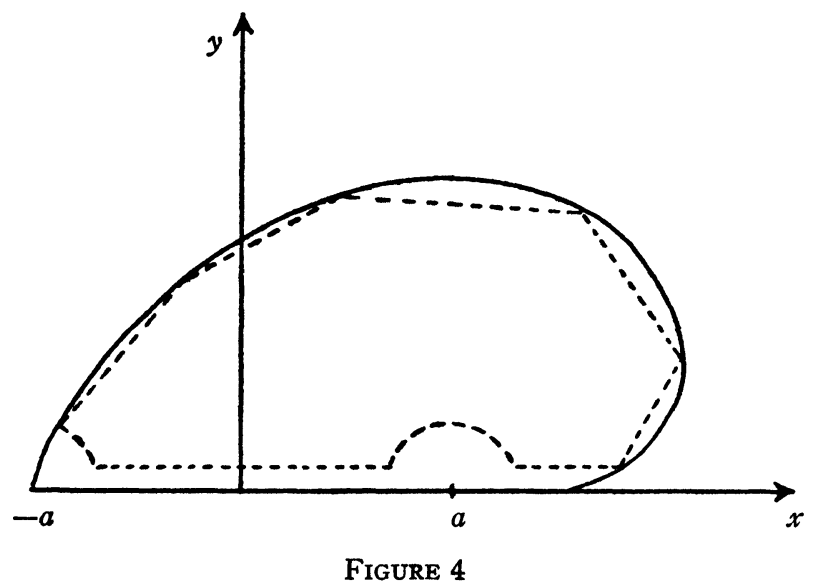

is larger than $8 y$. For every solution $z(x, y)$ of our problem the inequalities

$$
\begin{aligned}
& -q(x, y) \geqq \frac{d(x, y)}{6 y}, \quad\left|\frac{p(x, y)}{q(x, y)}\right| \leqq\left(\frac{12 y}{d(x, y)}\right)^{1 / 2}, \\
& \frac{-q(x, y)}{W(x, y)} \geqq 1-\frac{25 y}{d(x, y)}
\end{aligned}
$$

hold. Thus we have, uniformly for approach to any interval $|x|$ $\leqq a-\epsilon$ of $\partial P_{2}$ the relations $-q(x, y) \rightarrow \infty, p(x, y) / q(x, y) \rightarrow 0$, and $-q(x, y) / W(x, y) \rightarrow 1$.

The uniqueness proof now follows from (i) and (ii) applying the following lemma to a domain bounded by the dotted curve in $P$, arbitrarily close to $\partial P$, as indicated in Figure 4.

Let $R$ be a bounded domain whose boundary consists of finitely many piecewise smooth Jordan curves. Let $z_{1}(x, y), z_{2}(x, y) \in C^{2}(R) \cap C^{0}(\bar{R})$ be two solutions of the minimal surface equation in $R$ and set $W_{j}$ $=\left[1+p_{j}^{2}+q_{j}^{2}\right]^{1 / 2}$. For a compact subdomain $Q$ of $R$ denote by $m(Q)$ the maximum of $W_{1}$ and $W_{2}$ in $Q$. Then

$$
\frac{1}{2} \iint_{Q}\left[\left(p_{2}-p_{1}\right)^{2}+\left(q_{2}-q_{1}\right)^{2}\right] d x d y \leqq(2)^{1 / 2} m^{3}(Q) \oint_{\partial R}\left|z_{2}-z_{1}\right| d s .
$$

One last remark: Let $\left(x_{0}, y_{0}\right)$ be a fixed point of $P$ and denote by

$$
z^{*}(x, y)=\int_{\left(x_{0}, y_{0}\right)}^{(x, y)} \frac{p d y-q d x}{W}
$$


the conjugate function of the solution $z(x, y) . z^{*}(x, y)$ is continuous in $\bar{P}$. From (ii) it follows that

$$
z^{*}(a, 0)-z^{*}(-a, 0)=2 a .
$$

7.3. If one tries to solve a similar problem, where the solution is required to tend to $+\infty$ for approach of two or more straight segments of the boundary, new difficulties arise. We shall illustrate the situation with the case of two such segments, as indicated in Figure 5. On the open arcs $\partial P_{1}$ and $\partial P_{3}$ two bounded continuous functions $\phi_{1}$ and $\phi_{3}$ are given: $\left|\phi_{1}\right|,\left|\phi_{3}\right| \leqq M$. We ask for a solution $z(x, y)$ $\in C^{2}(P) \cap C^{0}\left(P \cup \partial P_{1} \cup \partial P_{3}\right)$ of the minimal surface equation in $P$

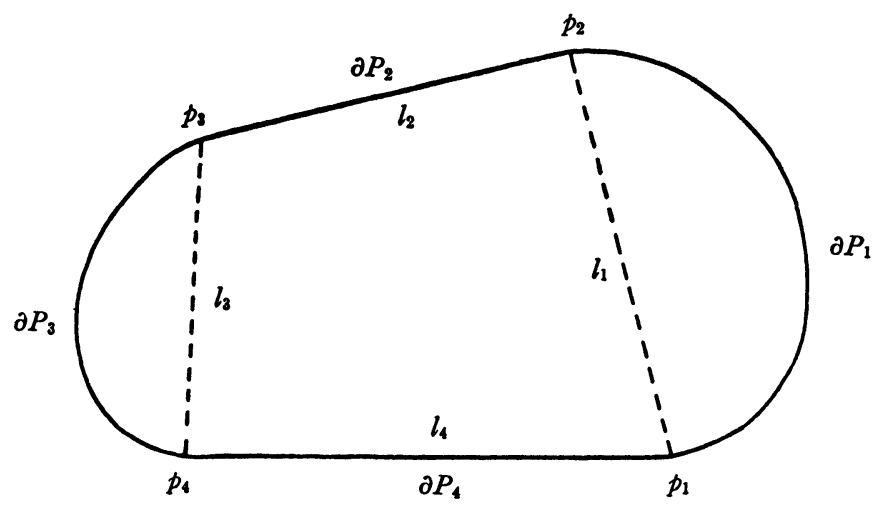

Figure 5

which assumes the values $\phi_{1}$ and $\phi_{3}$, respectively, on $\partial P_{1}$ and $\partial P_{3}$ and tends to $+\infty$ upon approach of every point of the open $\operatorname{arcs} \partial P_{2}$ and $\partial P_{4}$.

Let $z(x, y)$ be a solution of the problem. Denote by $l_{1}, l_{2}, l_{3}, l_{4}$, respectively, the lengths of the segments $p_{1} p_{2}, p_{2} p_{3}, p_{3} p_{4}, p_{4} p_{1}$ (see Figure 5). As before, we find that $z^{*}\left(p_{3}\right)-z^{*}\left(p_{2}\right)=l_{2}$ and $z^{*}\left(p_{1}\right)$ $-z^{*}\left(p_{4}\right)=l_{4}$ while, due to the inequality $p^{2}+q^{2}<W^{2}$, we will have $\left|z^{*}\left(p_{2}\right)-z^{*}\left(p_{1}\right)\right|<l_{1},\left|z^{*}\left(p_{4}\right)-z^{*}\left(p_{3}\right)\right|<l_{3}$. (If one of the arcs $\partial P_{1}$ or $\partial P_{3}$ coincides with the chord $p_{1} p_{2}$ or $p_{3} p_{4}$, the last inequalities require a more elaborate proof.) The continuity of $z^{*}(x, y)$ now implies the inequality $l_{2}+l_{4}<l_{1}+l_{3}$ as a necessary condition for the existence of a solution. It can be shown that this inequality is also a sufficient condition for the existence of a solution.

This interesting condition, found by $\mathrm{H}$. Jenkins and J. Serrin, which, at first glance, appears quite surprising, can easily be made understandable by a heuristic consideration. Let us try to obtain the solution $z(x, y)$ as limit of minimal surfaces $z(x, y ; n)$ which are equal 
to $\phi_{1}$ and $\phi_{3}$, respectively, on the $\operatorname{arcs} \partial P_{1}$ and $\partial P_{3}$ and equal to $n$ (where $n \gg M$ ) on the segments $\partial P_{2}$ and $\partial P_{4}$. For $n \rightarrow \infty$ two things may happen: Either the points of our minimal surfaces $z(x, y ; n)$ over the quadrilateral $p_{1} p_{2} p_{3} p_{4}$ are all "pulled up," or they are not "pulled up" (see also statement (iv) in II.13). In the first case the area of the minimal surface $z(x, y)$ would very roughly be equal to $l_{1} n+l_{3} n+\Delta$, where $\Delta$ is the area of the quadrilateral $p_{1} p_{2} p_{3} p_{4}$. In the second case the area of the minimal surface $z(x, y)$ would very roughly be equal to $l_{2} n+l_{4} n+\Delta+\delta$, where $\delta$ is the area of the part of $P$ outside the quadrilateral. Considering that our minimal surface will realize the absolute minimum of the area, the second possibility will occur if $l_{2}+l_{4}<l_{1}+l_{3}$ : The values of $z(x, y ; n)$ remain bounded as $n$ increases, and our solution $z(x, y)$ is obtained as limit for $n \rightarrow \infty$.

7.4. As an example of a case where the boundary values are partly $+\infty$ and partly $-\infty$ let us consider the following situation for the unit circle $P=\left\{x, y ; x^{2}+y^{2}<1\right\}$. Let $\partial P_{i}(i=1,2,3,4)$ be the arcs $\partial P_{i}=\{x=\cos \theta, y=\sin \theta ;(2 i-1) \pi / 4<\theta<(2 i+1) \pi / 4\}$. We have $l_{1}=l_{2}=l_{3}=l_{4}$. Denote by $z_{n}(x, y)(n=1,2, \cdots)$ the solutions of the minimal surface equation in $P$ with boundary values $-n$ on $\partial P_{1}$ and $\partial P_{3}$ and $+n$ on $\partial P_{2}$ and $\partial P_{4}$. Using the symmetry of the solution, which follows from the uniqueness and by which $z_{n}(x, x)=z_{n}(x,-x)$ $=0$, and the lemma of II.11 it is seen that the functions $z_{n}(x, y)$ converge for $n \rightarrow \infty$ to Scherk's minimal surface $\left(6^{\prime}\right)$ with $b=(2)^{1 / 2} / \pi$ in the square $P_{0}=\{x, y ;|x|,|y|<1\}$ and to $-\infty$ or $+\infty$ in the sets $P_{1}=\left\{x, y ; y \geqq 1 /(2)^{1 / 2}, x^{2}+y^{2}<1\right\}$, etc. By II.13 the convergence of the functions $z_{n}(x, y)$ and any of their derivatives to the solution $z(x, y)$ and the corresponding derivatives is uniform in every compact subdomain of $P_{0}$. Scherk's minimal surface is the unique solution of the minimal surface equation in $P_{0}$ which tends to $+\infty$ for approach of the vertical sides of $P_{0}$ and to $-\infty$ for approach of the horizontal sides of $P_{0}$.

8. Generalization of a lemma of T. Radó. Let $z_{1}(x, y)$ and $z_{2}(x, y)$ be different solutions of the minimal surface equation, defined in a neighborhood of the origin, and assume that $z_{1}(0,0)=z_{2}(0,0) . z_{1}(x, y)$ and $z_{2}(x, y)$ are real-analytic functions, and the difference $z_{2}(x, y)$ $-z_{1}(x, y)$ has an expansion $\sum_{i=1}^{\infty} P_{i}(x, y)$, where $P_{i}(x, y)$ is a homogeneous polynomial of degree $i$. As has been observed by W. H. Fleming [3, p. 80], J. Serrin [2], and G. Vaccaro [1], by substitution into (1), the first nonvanishing term $P_{j}(x, y)$ in this expansion is affinely related to a harmonic polynomial. From this fact it follows that a neighborhood of the origin is subdivided by $j \geqq 1$ smooth 
curves into $2 j \geqq 2$ open sectors $\sigma_{1}, \sigma_{2}, \cdots, \sigma_{2 j}$, such that $z_{2}(x, y)$ $<z_{1}(x, y)$ in $\sigma_{1}, \sigma_{3}, \cdots, \sigma_{2 j-1}$ and $z_{2}(x, y)>z_{1}(x, y)$ in $\sigma_{2}, \sigma_{4}, \cdots, \sigma_{2 j}$.

A similar but weaker statement (depending on the regularity properties of the coefficients $a, b, c$ ) can be proved for the general equation (2), if one remembers that the difference of two solutions of (2) satisfies itself a certain linear elliptic differential equation and uses some well-known facts about elliptic differential equations; see, for instance, R. Finn [6, p. 351], where a special case is treated.

An argument due to T. Radó [4, p. 793], complemented by the general maximum principle II.5.1, in which now the exceptional set consists of finitely many boundary points, immediately leads to the following useful lemma which, in special cases and with special proofs, was recently employed by R. Finn [6], J. Serrin [2], and R. Finn and R. Osserman [1]:

Given a Jordan domain $P$ in the $(x, y)$-plane and $2 n \geqq 2$ points $p_{j}(j=1,2, \cdots, 2 n)$ on its boundary $\partial P$, which bound $2 n$ open subarcs $\partial P_{1}, \partial P_{2}, \cdots, \partial P_{2 n}$. Let $z_{1}(x, y)$ and $z_{2}(x, y)$ be twice differentiable (and hence analytic) solutions of the minimal surface equation in $P$. Assume that lim sup $\left[z_{2}(x, y)-z_{1}(x, y)\right]<0$ for approach of every point on the arcs $\partial P_{1}, \partial P_{3}, \cdots, \partial P_{2 n-1}$ and lim inf $\left[z_{2}(x, y)-z_{1}(x, y)\right]>0$, for approach of every point on the arcs $\partial P_{2}, \partial P_{4}, \cdots, \partial P_{2 n}$. If, at a point $p_{0}$ of $P$ the two solutions $z_{1}(x, y)$ and $z_{2}(x, y)$ and all their derivatives of orders up to order $k(k \geqq 0)$ coincide, then the inequality $k \leqq n-1$ must hold.

For the proof consider the set of points in $P$, where $z_{2}(x, y)<z_{1}(x, y)$, and let $Q_{1}, Q_{2}, \cdots$ be its components. If a component $Q_{i}$ has a boundary point on an arc $\partial P_{2 j-1}$, by the assumptions this whole arc must belong to the boundary of $Q_{i}$. Denote by $Q^{(1)}, Q^{(3)}, \cdots, Q^{(2 n-1)}$ those of the components $Q_{1}, Q_{2}, \cdots$, which have, respectively, the $\operatorname{arcs} \partial P_{1}, \partial P_{3}, \cdots, \partial P_{2 n-1}$ as part of their boundary. We know that a neighborhood of the point $p_{0}$ is subdivided into $2(k+1)$ open sectors $\sigma_{1}, \sigma_{2}, \cdots, \sigma_{2 k+2}$, such that $z_{2}(x, y)<z_{1}(x, y)$ in $\sigma_{1}, \sigma_{3}, \cdots, \sigma_{2 k+1}$ and $z_{2}(x, y)>z_{1}(x, y)$ in $\sigma_{2}, \sigma_{4}, \cdots, \sigma_{2 k+2}$. Each component $Q^{(1)}$, $Q^{(3)}, \cdots, Q^{(2 n-1)}$ can have points in common with at most one of the sectors $\sigma_{1}, \sigma_{3}, \cdots, \sigma_{2 k+1}$. Otherwise, for topological reasons, a component of the set of points in $P$, where $z_{2}(x, y)>z_{1}(x, y)$, would have all its boundary points in $P$. Since $z_{2}(x, y)-z_{1}(x, y) \neq$ const, this is a contradiction to the maximum principle. For $k \geqq n$ there would be a sector $\sigma_{2 j-1}$ which would belong to a component $Q_{l}$ different from $Q^{(1)}, Q^{(3)}, \cdots, Q^{(2 n-1)}$. All its boundary points, with the possible exception of the points $p_{1}, p_{2}, \cdots, p_{2 n}$, would have to lie in $P$. By the general maximum principle this is not possible. Thus the assumption $k \geqq n$ leads to a contradiction. 


\section{Bernstein's theorem.}

9.1. This celebrated theorem, which for S. Bernstein was only a special consequence in a more general situation (see [4, Theorem 6 , p. 557]), but which has attracted considerable attention over the years, states that a minimal surface $z=z(x, y)$, defined for all values of $x$ and $y$, must be a plane. Bernstein's original proof contained a gap which was closed, independently, by E. Hopf [1] and E. J. Mickle [2]. Other proofs, partly valid for more general differential equations, have been given by T. Radó [2] and by L. Bers [2], [4], R. Finn [4], [5], W. H. Fleming [3, p. 83], E. Heinz [1], E. Hopf [2], H. Jenkins [1], K. Jörgens [1], J. C. C. Nitsche [1], [2], and R. Osserman [1], [4]. (Of course, many of the a priori estimates and general theorems to be discussed later imply Bernstein's theorem.)

By far the simplest of these proofs, due to J. C. C. Nitsche [1], [2], proceeds as follows: Introduce the functions

$$
\begin{aligned}
& \xi=\xi(x, y)=x+\int_{(0,0)}^{(x, y)} \frac{1+p^{2}}{W} d x+\frac{p q}{W} d y \equiv x+A(x, y), \\
& \eta=\eta(x, y)=y+\int_{(0,0)}^{(x, y)} \frac{p q}{W} d x+\frac{1+q^{2}}{W} d y \equiv y+B(x, y),
\end{aligned}
$$

in which the integrals are known to be path independent. Three facts are easily verified:

(i) The transformation $(x, y) \rightarrow(\xi, \eta)$ enlarges distances, hence maps the $(x, y)$-plane in one-to-one manner onto the $(\xi, \eta)$-plane.

(ii) $\xi$ and $\eta$ are isothermic parameters on the minimal surface.

(iii) The function $\Omega=(p-i q) /(1+W)$, already considered by S. A. Chaplygin, is analytic on the minimal surface, i.e., an analytic function of $\zeta=\xi+i \eta$. In fact, $\Omega(\zeta)=d\left(z+i z^{*}\right) / d \zeta$, where $z^{*}$ is the conjugate function of $z$; see II.7.2.

From $|\Omega(\zeta)|=[(W-1) /(W+1)]^{1 / 2}<1$ it follows by Liouville's theorem that $\Omega(\zeta)$ must be a constant. Thus $p=$ const, $q=$ const, i.e., the minimal surface must be a plane.

9.2. The integrands in (8) are nothing but the two differentials, discovered by $\mathrm{T}$. Radó [1],

$$
\left(F-q F_{q}\right) d x+q F_{p} d y, \quad p F_{q} d x+\left(F-p F_{p}\right) d y,
$$

which are exact for any solution of the Euler equation of a regular variational problem (5) (i.e., a problem satisfying $F_{p p} F_{q q}-F_{p q}^{2}>0$ ), or even for any $C^{1}$-solution of the Haar equations [1] associated with (5). Note that $F=W$ for the minimal surface equation.

The proof in J. C. C. Nitsche [1] shows, that property (i) of the 
mapping $(x, y) \rightarrow(\xi, \eta)$, associated with a variational problem whose integrand depends on the combination $\omega=\left(p^{2}+q^{2}\right)^{1 / 2}$ only, is guaranteed, if the condition

$$
F-p F_{q}-q F_{q}=F-\omega F^{\prime}>0,
$$

which already plays a role in the investigations of A. Haar [3] and E. J. Mickle [1], is satisfied. A similar condition handles the case of a general variational problem (5); see H. Jenkins [1].

Simply as property (i) can be proved-once its validity and usefulness in combination with properties (ii) and (iii) had been observedit was exploited in a number of papers; see, for instance, H. Jenkins [1], [2], H. Jenkins and J. Serrin [1], J. C. C. Nitsche [7], R. Osserman [4], J. Serrin [2, \$2]. In fact, property (i) is basic for all the estimates of these papers. Only the method recently designed by R. Finn and illustrated in II.9.4, II.12.1 (second part), and II.12.2, allows one to replace a number of the proofs using property (i) by alternate proofs.

We note that the line element and Gaussian curvature of the minimal surface $z=z(x, y)$ are given by the formulas

$$
\begin{aligned}
& d s=\frac{1}{2}\left(1+|\Omega(\zeta)|^{2}\right)|d \zeta| \\
& K=-\left|\Omega^{\prime}(\zeta)\right|^{2}\left(1+\frac{1}{W}\right)^{4}=-\frac{\partial(U, V)}{\partial(\xi, \eta)}\left(1+\frac{1}{W}\right)^{4} .
\end{aligned}
$$

Here we have set $\Omega=U+i V$; see J. C. C. Nitsche [1]. For the Gaussian curvature of a solution $z=z(x, y)$ of (5) one finds

$$
K=-\frac{\partial(U, V)}{\partial(\xi, \eta)}\left(\frac{1+F}{W}\right)^{4}
$$

Here we have set $\Omega=(p-i q) /(1+F)=U+i V$; see H. Jenkins [1, p. 713].

These similarities make it understandable that, under condition (10) and suitable further conditions on the integrand $F$, in conjunction with theorems on mappings of sufficiently slowly growing eccentricity $(\Omega(\zeta)$ is, in general, no longer an analytic function of $\zeta)$, Bernstein's theorem, and other theorems as well, hold for solutions of (5).

9.3. While the first proofs of Bernstein's theorem were qualitative in nature, E. Heinz and F. Rellich gave a quantitative proof, based on certain a priori estimates by E. Heinz [1]:

Let $z=z(x, y)$ be a minimal surface, defined over the disc $x^{2}+y^{2}<R^{2}$. 
The value $K_{0}$ of its Gaussian curvature at the origin satisfies the inequality

$$
\left|K_{0}\right| \leqq \frac{c_{1}}{R^{2}}, \quad c_{1}=\frac{4 \pi^{3}}{3} .
$$

Inequality (13) was sharpened by E. Hopf [2] and improved by J. C. C. Nitsche [1], [7] and R. Osserman [4]:

Let $z=z(x, y)$ be a minimal surface, defined over the disc $x^{2}+y^{2}<R^{2}$. Then

$$
\left|K_{0}\right| \leqq \frac{c_{2}}{W_{0}^{2} R^{2}} .
$$

For the proof, of which the following version is due to R. Osserman [4], denote by $\Pi$ the image of the disc $P=\left\{x, y ; x^{2}+y^{2}<R^{2}\right\}$ under the mapping (8) and by $\rho R$ the distance of the origin in the $\zeta$-plane from the complement of $\Pi$. Let $\zeta_{0}$ be a point of this complement, for which $\left|\zeta_{0}\right|=\rho R, \lambda$ the half-open segment $\left\{\zeta ; \zeta=t \zeta_{0}, 0 \leqq t<1\right\}$, and $l$ its image in the $(x, y)$-plane under the inverse mapping of $(8)$. By (11)

$$
R \leqq \int_{l} d s=\frac{1}{2} \int_{\lambda}\left(1+|\Omega(\zeta)|^{2}\right)|d \zeta|
$$

Since $|\Omega(\zeta)|<1$ in $|\zeta|<\rho R$ (even in $\Pi$ ), we have

$$
|\Omega(\zeta)| \leqq(\rho R a+|\zeta|)(\rho R+a|\zeta|)^{-1}
$$

where

$$
a=|\Omega(0)|=\left[\left(W_{0}-1\right) /\left(W_{0}+1\right)\right]^{1 / 2},
$$

and hence

$$
1 \leqq \frac{\rho}{2} \int_{0}^{1}\left[1+\left(\frac{a+t}{1+a t}\right)^{2}\right] d t \equiv \rho f(a) .
$$

On the other hand, $\left|\Omega^{\prime}(0)\right| \leqq\left(1-a^{2}\right) / \rho R$ by the Schwarz-Pick lemma. By (11)

$$
\left|K_{0}\right|=\left|\Omega^{\prime}(0)\right|^{2}\left(1+\frac{1}{W_{0}}\right)^{4} \leqq \frac{1}{W_{0}^{2} R^{2}}\left[\frac{4 f(a)}{1+a^{2}}\right]^{2} .
$$

Thus we certainly have

$$
c_{2} \leqq \operatorname{Max}_{0 \leqq a \leqq 1}\left[\frac{4 f(a)}{1+a^{2}}\right]^{2}=7.70145 \cdots
$$

9.4. An interesting new proof of the inequalities (13), (14), based on an idea of R. Finn, was recently given by R. Finn and R. Osserman [1]. We present it here in a somewhat simplified version. With- 
out loss of generality, it can be assumed that $p_{0}=p(0,0) \geqq 0, q_{0}(0,0)$ $\geqq 0, r(0,0) \geqq 0, s(0,0)=0, t(0,0)=-r(0,0)\left(1+q_{0}^{2}\right) /\left(1+p_{0}^{2}\right) \leqq 0$. These relations can be realized by carrying out, if necessary, a rotation and some of the following replacements: $x$ by $y$ and $y$ by $x, x$ by $-x$, $y$ by $-y$. None of these transformations changes the value of the Gaussian curvature. In view of a later application (in II.12.1) we note that none of these transformations changes the value of the combination $r^{2}+2 s^{2}+t^{2}$ either.

Consider Scherk's minimal surface

$$
\begin{array}{r}
z=\phi(x, y ; h)=h\left\{\log \cos \left(\frac{y-\beta(h)}{h}\right)-\log \cos \left(\frac{x+\alpha(h)}{h}\right)\right\}, \\
\alpha(h)=h \tan ^{-1} p_{0}, \quad \beta(h)=h \tan ^{-1} q_{0},
\end{array}
$$

defined over the oriented square

$$
R_{h}=\{x, y ;|x+\alpha(h)|<\pi h / 2,|y-\beta(h)|<\pi h / 2\},
$$

whose first derivatives at the origin coincide with those of the solution $z(x, y) . R_{h}$ is contained in $P$ as long as

$$
h \leqq h_{0}=\frac{2 R}{\pi}\left[\left(1+\frac{2}{\pi} \tan ^{-1} p_{0}\right)^{2}+\left(1+\frac{2}{\pi} \tan ^{-1} q_{0}\right)^{2}\right]^{-1} .
$$

The Gaussian curvature $\Re(x, y ; h)$ of the above surface, taken at the origin, is equal to

$$
\Re(0,0 ; h)=-\frac{1}{h^{2}} \frac{\left(1+p_{0}\right)^{2}\left(1+q_{0}^{2}\right)}{\left(1+p_{0}^{2}+q_{0}^{2}\right)^{2}} .
$$

We assert that the inequality $\left|K_{0}\right| \leqq\left|\Omega\left(0,0 ; h_{0}\right)\right|$ holds. Since $|\Omega(0,0 ; h)|$ increases with decreasing $h$ (otherwise a number $h_{1}$ $\left(0<h_{1}<h_{0}\right)$ could be found so that $\left|K_{0}\right|=\left|\Omega\left(0,0 ; h_{1}\right)\right|$, i.e., $r(0,0)$ $=\phi_{x x}\left(0,0 ; h_{1}\right), s(0,0)=\phi_{x y}\left(0,0 ; h_{1}\right)=0, t(0,0)=\phi_{y y}\left(0,0 ; h_{1}\right)$ (this follows from the fact that both functions satisfy the minimal surface equation); by the lemma of II.8 this is impossible), we get

$$
\begin{aligned}
& R^{2} W_{0}^{2}\left|K_{0}\right| \\
& \quad \leqq \frac{\pi^{2}}{4} \frac{\left(1+p_{0}^{2}\right)\left(1+q_{0}^{2}\right)}{1+p_{0}^{2}+q_{0}^{2}}\left[\left(1+\frac{2}{\pi} \tan ^{-1} p_{0}\right)^{2}+\left(1+\frac{2}{\pi} \tan ^{-1} q_{0}\right)^{2}\right] \\
& \quad \leqq \frac{\pi^{2}}{8} \frac{\left(1+W_{0}^{2}\right)}{W_{0}^{2}}\left[1+\frac{2}{\pi} \tan ^{-1}\left(\left(\frac{\left(p_{0}^{2}+q_{0}^{2}\right)}{2}\right)^{1 / 2}\right)\right]^{2} \\
& \quad \leqq \frac{\pi^{2}}{2}\left(\frac{1+a^{4}}{1-a^{4}}\right)^{2}\left[1+\frac{2}{\pi} \tan ^{-1}\left(\frac{(2)^{1 / 2} a}{1-a^{2}}\right)\right]^{2} \equiv g(a) .
\end{aligned}
$$


Here $a$ has the same meaning as in II.9.3.

In order to combine the last two inequalities, let us set $h(a)$ $=\operatorname{Min}(f(a), g(a))$. A computation shows that

$$
c_{2} \leqq \operatorname{Max}_{0 \leqq a} h(a)=7.678447 \cdots
$$

9.5. The best values of the constants $c_{1}$ and $c_{2}$, i.e., the values

$$
c_{1}^{(0)}=\operatorname{Sup}\left|R^{2} K_{0}\right|, \quad c_{2}^{(0)}=\operatorname{Sup}\left|R^{2} W_{0}^{2} K_{0}\right|,
$$

where the supremum is taken over all minimal surfaces $z=z(x, y)$ in the circle $x^{2}+y^{2}<R^{2}$, have so far defied all attempts at their determination. Clearly $c_{1}^{(0)} \leqq c_{2}^{(0)}$.

A lower bound is easily obtained if one considers the minimal surfaces $z_{n}(x, y)$ of II.7.4. By the uniform convergence the Gaussian curvature of the surfaces $z_{n}(x, y)$, taken at the origin, converges to the Gaussian curvature of Scherk's minimal surface, taken at the origin. The latter has the value $\pi^{2} / 2$. So we find

$$
\begin{aligned}
& \pi^{2} / 2=4.934807 \cdots \leqq c_{1}^{(0)} \leqq 5.921407 \cdots, \\
& \pi^{2} / 2=4.934807 \cdots \leqq c_{2}^{(0)} \leqq 7.678447 \cdots \cdot
\end{aligned}
$$

\section{Harmonic mappings and related questions.}

10.1. Since the components of the vector of a minimal surface are harmonic functions on the surface, the inverse functions $x=x(\xi, \eta)$, $y=y(\xi, \eta)$ of the transformation (8) are harmonic functions. Properties of harmonic mappings, i.e., mappings by (generally nonconjugate) harmonic functions, have played a role in the investigations of T. Radó [2], H. Lewy [1], L. Bers [2], E. Heinz [1], [4], E. Hopf [2], K. Jörgens [2], J. C. C. Nitsche [5], [8], [11], [14], R. M. Redheffer [1], H. L. de Vries [1], J. L. Ullman and C. J. Titus [1], K. Shibata [1] and others. Harmonic mappings in more than two dimensions raise serious questions; see H. Lewy [3], B. Segre [1], [2].

Let us consider the class $\mathfrak{S}$ of one-to-one harmonic mappings $x=x(\xi, \eta), y=y(\xi, \eta)$ of the unit disc $\xi^{2}+\eta^{2} \leqq 1$ onto the unit disc $x^{2}+y^{2} \leqq 1$, leaving the origin fixed, i.e., $x(0,0)=y(0,0)=0$. The functions $x(\xi, \eta)$ and $y(\xi, \eta)$ are assumed to be harmonic in $\xi^{2}+\eta^{2}<1$ and continuous in $\xi^{2}+\eta^{2} \leqq 1$. While there are-contrary to the situation for conformal mappings, where $x(\xi, \eta)$ and $y(\xi, \eta)$ are conjugate harmonic functions-mappings of class $\mathfrak{S}$, for which the Jacobian $J(\xi, \eta)=\partial(x, y) / \partial(\xi, \eta)$ (by a theorem of H. Lewy [1], $J(\xi, \eta)$ cannot vanish and therefore may be assumed to be positive), taken at the origin, is arbitrarily small, it has been shown by E. Heinz [1] that 
the expression $\phi(\xi, \eta)=x_{\xi}^{2}+x_{\eta}^{2}+y_{\xi}^{2}+y_{\eta}^{2}$, taken at the origin, can be bounded from below by a universal constant

$$
c_{3}^{(0)}=\operatorname{Inf}_{\mathfrak{G}} \phi(0,0)
$$

The value of this constant is not known. Numerical estimates have been given by E. Heinz [1], J. C. C. Nitsche [5], [8], [14], and H. L. de Vries [1]. The best estimate at present is $c_{3}^{(0)} \geqq 0.8952$ (see J. C. C. Nitsche [14]). A similar inequality holds also for more general univalent mappings; see P. Berg [1], E. Heinz [2], [3]. E. Heinz [4] also proved that $\phi(\xi, \eta) \geqq 2 / \pi^{2}$, uniformly in $\xi^{2}+\eta^{2}<1$. Again, the value of the constant

is not known.

$$
c_{4}^{(0)}=\operatorname{Inf}_{\mathfrak{S}}\left\{\operatorname{Inf}_{\xi^{2}+\eta^{2}<1} \phi(\xi, \eta)\right\}
$$

Applying the above inequality to the estimation (13) of the Gaussian curvature, E. Heinz found that $c_{1}^{(0)} \leqq 8 / c_{3}^{(0)}$.

J. L. Ullman and C. J. Titus [1] have estimated the expression $\Psi(\xi, \eta) \equiv \phi(\xi, \eta)+2 J(\xi, \eta)$, taken at the origin, for mappings of a subclass $\mathfrak{S}_{1}$ of $\mathfrak{W}$. It is easy to see that, in the notation of J. C. C. Nitsche [5] (merely replacing $\gamma=\alpha+i \beta$ by $\zeta=\xi+i \eta), \Psi(\xi, \eta)=\left|W^{\prime}(\zeta)\right|^{2}$. If we set

$$
c_{\mathfrak{S}}^{(0)}=\operatorname{Inf}_{\mathfrak{S}}|\Psi(0,0)|,
$$

it is then clear that $c_{5}^{(0)} \geqq 1$. However, the exact value of $c_{5}^{(0)}$ is not known. Ullman and Titus conjecture that $c_{5}^{(0)}=16 / \pi^{2}$ (see $[1$, p. 187]) and prove this equality for the subclass $\mathfrak{S}_{1}$. The number $16 / \pi^{2}$, which is the limit value of $\phi(0,0)$ and $\Psi(0,0)$ for a certain one-parameter family of harmonic mappings, had already earlier played a role; see E. Hopf [2, p. 522 and p. 801] and J. C. C. Nitsche [5, pp. 270-271]. One should also try to determine the constant

$$
c_{6}^{(0)}=\operatorname{Inf}_{\mathscr{S}}\left\{\operatorname{Inf}_{\xi^{2}+\eta^{2}<1} \Psi(\xi, \eta)\right\} .
$$

A lemma in the same spirit is the following (see J. C. C. Nitsche [11]):

Denote by $\Re(\rho)$ the class of all one-to-one harmonic mappings of the annulus $0<\rho^{2}<\xi^{2}+\eta^{2}<1$ onto an annulus $0<r^{2}<x^{2}+y^{2}<1$. Let $R(\rho)$ be the least upper bound for all inner radii $r$, obtainable by mappings of this class. Then $R(\rho)<1$.

The exact value of $R(\rho)$ is not known at present. It is my conjecture that $R(\rho)=2 \rho /\left(1+\rho^{2}\right)$. 
10.2. Let $z(x, y)$ be a solution of the minimal surface equation in a simply connected domain $P$. Since the functions $A(x, y)$ and $B(x, y)$ of (8) satisfy the relations $A_{y}=B_{x}$ and $A_{x} B_{y}-A_{y} B_{x}=1$, there is a function $Z(x, y)$ such that $A=Z_{x}, B=Z_{y}$, and the minimal surface equation is closely related to the Monge-Ampère differential equation

$$
Z_{x x} Z_{y y}-Z_{x y}^{2}=1
$$

We remark that in a similar fashion a connection between a regular variational problem, with integrand $F(p, q)$ depending only on the combination $\omega=\left(p^{2}+q^{2}\right)^{1 / 2}$ and satisfying (10), and the differential equation.

$$
Z_{x x} Z_{y y}-Z_{x y}^{2}=f\left(Z_{x x}+Z_{y y}\right)
$$

can be established. This relationship has not yet been exploited.

Bernstein's theorem of II.9 is a consequence of the theorem that a solution of (22), defined for all $x$ and $y$, must be a quadratic polynomial. This theorem was first proved by K. Jörgens [1], using the lemma of E. Heinz on harmonic mappings. A short elementary proof was given by J. C. C. Nitsche [2]. This proof is based on the property of the mapping $\xi=x+Z_{x}, \eta=y+Z_{y}$, which was invented by $\mathrm{H}$. Lewy [2] for other purposes, to enlarge distances, and on the fortunate fact that the complex-valued function $\left(x-Z_{x}\right)+i\left(Z_{y}-y\right)$, in its dependence on $\xi$ and $\eta$, is an analytic function of $\xi=\xi+i \eta$ with bounded derivative.

Isolated singularities of solutions of the differential equation (22) and its connection with harmonic mappings have been investigated by K. Jörgens [2]; see also J. C. C. Nitsche [5]. Generalizations to higher dimensions, in different directions, have been given by $\mathrm{E}$. Calabi [1] and H. Flanders [1]. The relation between the differential equation (22), two-dimensional Finsler spaces, and especially the twodimensional elliptic geometry has been uncovered and utilized by P. Funk [1], [2].

11. Consequences of the maximum principle. Comparison of the solution $z(x, y)$ with certain auxiliary functions-solutions or super solutions of the minimal surface equation whose values or normal derivatives become infinite on certain parts of the boundary (Scherk's minimal surface (6), the surface (7), the functions $\phi_{i}$ of II.2.3, the function used by J. C. C. Nitsche [6], etc.)-leads to various elementary but useful facts. Some have already been discussed in II. 2 and II.5.2. Another, proved by H. Jenkins and J. Serrin [1, p. 206] is the following: 
Let $A$ and $B$ be complementary open subarcs of the boundary $\partial P$ of $a$ bounded convex domain in the $(x, y)$-plane and $\bar{R}$ the (closed) convex hull of $B$.

Let $z(x, y)$ be a solution of the minimal surface equation in $P$, and assume that $\lim \sup z(x, y) \leqq M(\lim \inf z(x, y) \geqq m)$ for approach of any point on $A$. For any compact subset $T$ of $P-\bar{R}$ there is a number $N$, depending on this subset, but independent of the solution $z(x, y)$, such that the inequalities $z(x, y) \leqq M+N(z(x, y) \geqq m-N)$ hold in all points of $T$.

Denote by $z_{k}(x, y)$ the solution of the general Dirichlet problem in $P$ with boundary values 0 on $A$ and $k>0$ on $B$. Denote by $\bar{Q}$ and $\bar{R}$ the closed convex hulls of the $\operatorname{arcs} A$ and $B$, respectively. We are interested in the behavior of the functions $z_{k}(x, y)$ if $k$ tends to infinity. By the preceding there is a function $N=N(x, y)$, defined and finite in $\bar{P}-\bar{Q} \cap \bar{R}$, such that the inequalities

$$
\begin{array}{ll}
z_{k}(x, y) \leqq N(x, y) & \text { for }(x, y) \in P-\bar{R}, \\
z_{k}(x, y) \geqq k-N(x, y) & \text { for }(x, y) \in P-\bar{Q}
\end{array}
$$

hold independent of $k$. We shall use this fact in II.13.

Observe that, for harmonic functions, or for solutions of uniformly elliptic equations, such a behavior is impossible by Harnack's inequality.

For further theorems in this spirit see H. Jenkins and J. Serrin [1] and R. Finn [6], [7].

12. A priori estimates. Many of the details of the investigations discussed earlier depend upon a priori estimates for solutions of the minimal surface equation. Many such estimates have been improved, and new ones have been discovered, in recent years.

12.1. Estimates of the second derivatives by the first. Estimates of the second derivatives in terms of bounds on the solution and its first derivatives have first been given by C. H. Müntz [1] and, later, in a more explicit form and in terms of the first derivatives alone, by E. Heinz [1] (with power $W_{0}^{6}$ in (24)). A somewhat sharper estimate is contained in the theorem (proved by J. C. C. Nitsche [4]; see also H. Jenkins [1, p. 198]):

Let $z(x, y)$ be a solution of the minimal surface equation over the circle $x^{2}+y^{2}<R^{2}$. Then

$$
r_{0}^{2}+2 s_{0}^{2}+t_{0}^{2} \leqq \frac{c_{7}}{R^{2}} W_{0}^{4}
$$


Differentiating the relation $p-i q=2 \Omega /\left(1-|\Omega|^{2}\right)$, we find

$$
r-i s=\frac{\partial}{\partial x}\left(\frac{2 \Omega}{1-|\Omega|^{2}}\right)=\left(\zeta_{x} \frac{\partial}{\partial \zeta}+\bar{\zeta}_{x} \frac{\partial}{\partial \bar{\zeta}}\right)\left(\frac{2 \Omega}{1-|\Omega|^{2}}\right)
$$

and an analogous expression for $s-i t$. Estimating again, as in II.9.3, the derivative $\Omega^{\prime}(\zeta)$ by the Schwarz-Pick lemma, we obtain, after a simple computation,

The best value

$$
r_{0}^{2}+2 s_{0}^{2}+t_{0}^{2} \leqq \frac{8}{R^{2}} W_{0}^{2}\left(1+W_{0}\right)^{2} \leqq \frac{32}{R^{2}} W_{0}^{4} .
$$

$$
c_{6}^{(0)}=\operatorname{Sup}\left\{R^{2} W_{0}^{-4}\left(r_{0}^{2}+2 s_{0}^{2}+t_{0}^{2}\right)\right\},
$$

where the supremum is taken over all minimal surfaces $z=z(x, y)$ in the disc $x^{2}+y^{2}<R^{2}$, is not known. However, a better numerical bound is found using the estimation of II.9.4. With the normalization (remembering the remark at the beginning) and the notations of this paragraph it follows from $\left|K_{0}\right| \leqq\left|\Omega\left(0,0 ; h_{0}\right)\right|$ and the fact that $z$ and $\phi$ are solutions of the minimal surface equation, that $r_{0} \leqq \phi_{x x}\left(0,0 ; h_{0}\right)$, $\left|t_{0}\right| \leqq\left|\phi_{y y}\left(0,0 ; h_{0}\right)\right|$ and, thus,

$$
\begin{aligned}
& r_{0}^{2}+2 s_{0}^{2}+t_{0}^{2} \\
& \quad \leqq \phi_{x x}^{2}\left(0,0 ; h_{0}\right)+\phi_{y y}^{2}\left(0,0 ; h_{0}\right)=\frac{1}{h_{0}^{2}}\left[\left(1+p_{0}^{2}\right)^{2}+\left(1+q_{0}^{2}\right)^{2}\right] \\
& \leqq \frac{\pi^{2} W_{0}^{4}}{4 R^{2}} \frac{\left(1+p_{0}^{2}\right)^{2}+\left(1+q_{0}^{2}\right)^{2}}{W_{0}^{4}}\left[\left(1+\frac{2}{\pi} \tan ^{-1} p_{0}\right)^{2}+\left(1+\frac{2}{\pi} \tan ^{-1} q_{0}\right)^{2}\right] \\
& \equiv \frac{W_{0}^{4}}{R^{2}} f\left(p_{0}, q_{0}\right) .
\end{aligned}
$$

An analysis of the function $f(p, q)$ shows that $f(p, q) \leqq 2 \pi^{2}$. Hence $c_{7}^{(0)} \leqq 2 \pi^{2}$. An argument similar to that in II.9.5 shows that $c_{7}^{(0)} \geqq \pi^{2}$. Thus

$$
\pi^{2} \leqq c_{7}^{(0)} \leqq 2 \pi^{2} .
$$

12.2. Estimates of the first derivatives by the solution. Such estimates were first given by R. Finn (see [1] and [3, p. 410]) for bounded solutions and have since been improved and generalized by $\mathrm{H}$. Jenkins and J. Serrin [1] and R. Finn [6] to positive solutions. Recently J. Serrin [2] gave a particularly short proof. It is based on an idea of 
R. Finn which, applied to a different question, has already been described in II.9.4.

Let $z(x, y)$ be a positive solution of the minimal surface equation over the circle $x^{2}+y^{2}<R^{2}$. Then

$$
W_{0} \leqq c_{8} e^{\pi z_{0} / 2 R} \quad\left(z_{0}=z(0,0)\right) .
$$

We shall give a simplified and slightly improved version of Serrin's proof. Without loss of generality, it may be assumed that $p_{0} \geqq 0$, $q_{0}=0$. Consider the Scherk minimal surface

$$
\begin{aligned}
z & =\phi(x, y ; a)=h(a)\left\{\log \cos \left(\frac{y}{h(a)}\right)-\log \cos \left(\frac{x+a}{h(a)}\right)\right\}, \\
h(a) & =\left(\frac{a+\left(2 R^{2}-a^{2}\right)^{1 / 2}}{\pi}\right), \quad 0<a<R,
\end{aligned}
$$

over the triangle $R_{a}=\{x, y ; 0 \leqq x+a \leqq|y|,|y|<\pi h(a) / 2\}$. This triangle contains the origin $x=y=0$ and is contained in the circle $x^{2}+y^{2}<R^{2}$. We shall choose the parameter $a$ in such a way that $\phi(0,0 ; a)=z_{0}$, i.e.,

$$
\cos (a / h(a))=e^{-z_{0} / h(a)} .
$$

This transcendental equation has exactly one root $a=a_{0}$ in the interval $0<a<R$.

Similarly as in II.9.4 we now assert that $p_{0} \leqq \phi_{x}\left(0,0 ; a_{0}\right)$ (remember that $\left.q_{0}=\phi_{y}(0,0 ; a)=0\right)$. Since $\phi_{x}(0,0 ; a)=\tan (a / h(a))$ increases monotonically to $+\infty$ as $a$ increases from 0 to $R$, there would otherwise exist a number $a_{1}\left(a_{0}<a_{1}<R\right)$ such that $\phi_{x}\left(0,0 ; a_{1}\right)=p_{0}$. By the lemma of II. 8 this is impossible. Thus we find

$$
W_{0}=\left(1+p_{0}^{2}\right)^{1 / 2} \leqq\left(1+\phi_{x}^{2}\left(0,0 ; a_{0}\right)\right)^{1 / 2}=e^{z_{0} / h\left(a_{0}\right)}
$$

and, by the equation relating $z_{0}$ and $a$,

$$
W_{0} \leqq e^{\pi z_{0} / 2 R} \exp \left\{\left(\frac{\pi h\left(a_{0}\right)}{2 R}-1\right) \log \cos \left(\frac{a_{0}}{h\left(a_{0}\right)}\right)\right\} \equiv f\left(a_{0}\right) e^{\pi z_{0} / 2 R} .
$$

A computation shows that $\operatorname{Sup}_{0<a<1} f(a)=1.03894$. Thus $(27)$ is proved with $c_{8}=1.03894$.

Clearly, (27) implies an estimate of the gradient by the solution for bounded solutions:

Let $z(x, y)$ be a solution of the minimal surface equation in the circle $x^{2}+y^{2}<R^{2}$, and assume that $|z(x, y)| \leqq M$. Then

$$
W_{0} \leqq c_{9} e^{\pi M / 2 R} \text {. }
$$

Here we certainly can take $c_{9} \leqq c_{8} \exp \left\{-\pi\left|z_{0}\right| / 2 R\right\}$. By an example 
of R. Finn [6, p. 355] it is seen that the exponent $\pi / 2$ in (27) cannot be improved. That the factor $\pi / 2$ in the exponent in (28) is the right one, was first indicated by a heuristic consideration of J. B. Keller [1]. In the class of all solutions $z(x, y)$ of the minimal surface equation, defined in the circle $P=\left\{x, y ; x^{2}+y^{2}<R^{2}\right\}$ and satisfying the conditions $z(0,0)=0,|z(x, y)|<M$, the solution which maximizes the expression $W_{0}$ is characterized as solution of the generalized Dirichlet problem II.3 which is equal to $+M$ on an open subarc of $\partial P$ of length $\pi R$ and equal to $-M$ on the complementary (open) subarc of $\partial P$. The best value of the constant $c_{8}$, i.e.,

$$
c_{8}^{(0)}=\operatorname{Sup}\left\{W_{0} e^{-\pi z_{0} / 2 R}\right\}
$$

the supremum taken over all positive solutions of the minimal surface equation in the disc $x^{2}+y^{2}<R^{2}$, is not known.

J. Serrin [2] (see also H. Jenkins and J. Serrin [1, p. 200] and R. Finn. [6]) proved also the following form of the estimate (27) for small values of $z(0,0)$ :

Let $z(x, y)$ be a positive solution of the minimal surface equation in the circle $x^{2}+y^{2}<R^{2}$. For every $\epsilon>0$ there is a number $\delta=\delta(\epsilon)>0$ such that

for $z_{0} \leqq R \delta(\epsilon)$.

$$
\left(p_{0}^{2}+q_{0}^{2}\right)^{1 / 2} \leqq \frac{2+\epsilon}{R} z_{0}
$$

12.3. Estimates of the first derivatives by the area.

Let $z(x, y)$ be a solution of the minimal surface equation in the disc $x^{2}+y^{2}<R^{2}$ and $I(S)$ the area of the minimal surface $S$ defined by $i t$. Then

$$
W_{0} \leqq \frac{16 I(S)}{\pi R^{2}} e^{4 \pi I(S) / R} .
$$

This estimate (with exponent $64 \pi I(S) / R^{2}$ ) was derived by R. Finn (see [1] and [3, p. 408] and corrections of [3] in [8]), using properties of topological mappings with bounded Dirichlet integral (for such mappings, see J. Lelong-Ferrand [1, especially Chapter II]) in combination with a lemma of function theory, applied to the function $\Omega^{2}(\zeta)$, which we shall phrase here in the following way (Lemma 1 , as it stands in R. Finn [3], is not quite correct; see above): Let $f(z)$ be analytic and $|f(z)|<1$ in $|z|<1$. Then, for $|z| \leqq \rho<1$, the inequality

$$
|f(z)| \geqq \operatorname{Max}\left\{0, \frac{\left(1+\rho^{2}\right) M_{\rho}-2 \rho}{1+\rho^{2}-2 \rho M_{\rho}}\right\}
$$

holds. Here $M_{\rho}=\operatorname{Max}_{|z| \leq \rho}|f(z)|$. 
12.4. Estimate of the area by the $L^{1}$-norm of the boundary values.

Let $z(x, y) \in C^{2}(P) \cap C^{0}(\bar{P})$ be a solution of the minimal surface equation in a region $P$ of area $|P|$, bounded by a rectifiable Jordan curve $\partial P$, and $S$ the minimal surface defined by $z(x, y)$. For any constant $c$, the inequality

$$
I(S) \leqq|P|+\int_{\partial P}|z-c| d s
$$

holds.

For the proof, approximate $\partial P$ from the interior by analytic Jordan curves $\mathcal{C}_{n}(n=1,2, \cdots)$ with interiors $P_{n}$. If $S_{n}$ denotes the part of $S$ over $P_{n}$, then

$$
I\left(S_{n}\right)=\iint_{P_{n}} W d x d y=\iint_{P_{n}} W^{-1} d x d y+\iint_{P_{n}} W^{-1}\left(p^{2}+q^{2}\right) d x d y .
$$

The first integral on the right is smaller than $|P|$. For the second integral we have

$$
\begin{aligned}
& \iint_{P_{n}} W^{-1}\left(p^{2}+q^{2}\right) d x d y \\
& \quad=\iint_{P_{n}}\left[\left((z-c) p W^{-1}\right)_{x}+\left((z-c) q W^{-1}\right)_{y}\right] d x d y \\
& \quad=\int_{\mathcal{C}_{n}} W^{-1}(z-c)[p d y-q d x] \leqq \int_{\mathfrak{C}_{n}}|z-c| d s .
\end{aligned}
$$

Inequality (32) follows for $n \rightarrow \infty$.

12.5. Estimate of the solution by the $L^{1}$-norm of its boundary values. We state the following theorem without proof.

Let $z(x, y) \in C^{2}(P) \cap C^{0}(\bar{P})$ be a solution of the minimal surface equation in a bounded convex domain $P$. Denote by $P_{d}$ the subset of all points of $P$ whose distance from $\partial P$ is not less than $d>0$. There exists a constant $M$, depending on $d, P$, and $\int_{\partial P}|z| d s$ only, such that the inequality $|z(x, y)| \leqq M$ holds in $P_{d}$.

(Explicit bounds for the constant $M$ can be given.) This estimate allows us, by approximation arguments, once more generalizing Dirichlet's problem beyond the stage discussed in II.3, to prove the existence of a solution of Dirichlet's problem for the minimal surface equation in a convex (not necessarily strictly convex) domain with boundary data which, as functions of the arc length of the boundary, are merely absolutely integrable.

The uniqueness proof, however, hinges on an estimate of the kind formulated in problem 21 of Chapter VII.

12.6. Harnack's inequality. Integrating inequalities (27) and (30), 
H. Jenkins and J. Serrin [1, p. 203] obtain a Harnack-type inequality, valid for the minimal surface equation and more general equations. This inequality sheds light on the situation described in II.11. Note again the sharp difference with respect to the situation for harmonic functions and uniformly elliptic equations, where a bound on $z_{0}=z(0,0)$ implies a uniform bound in every compact subdomain of $x^{2}+y^{2}<R^{2}$.

Let $\boldsymbol{z}(x, y)$ be a positive solution of the minimal surface equation in the circle $x^{2}+y^{2}<R^{2}$. There are two functions $Q_{1}(t ; \mu)$ and $Q_{2}(t ; \mu)$ such that

$$
R Q_{1}\left(\frac{r}{R} ; \frac{z_{0}}{R}\right) \leqq z(x, y) \leqq R Q_{2}\left(\frac{r}{R} ; \frac{z_{0}}{R}\right),
$$

$r=\left(x^{2}+y^{2}\right)^{1 / 2}$. The functions $Q_{1}$ and $Q_{2}$ have the following properties:

For each $\mu \geqq 0, Q_{1}(t ; \mu)$ is a continuous and monotonically decreasing function of $t$ in $0 \leqq t \leqq 1$, and $Q_{1}(0 ; \mu)=\mu, Q_{1}(1 ; \mu)=0$.

For each $\mu>0, Q_{2}(t ; \mu)$ is a continuous and monotonically increasing function of $t$ in an interval $0 \leqq t<\rho(\mu)<1$, and $Q_{2}(0 ; \mu)=\mu$, $\lim _{t \rightarrow \rho(\mu)} Q_{2}(t ; \mu)=\infty$. Here $\rho(\mu)$ is a continuous and monotonically decreasing function in $0<\mu<\infty$ and $\lim _{\mu \rightarrow 0} \rho(\mu)=1, \lim _{\mu \rightarrow \infty} \rho(\mu)=0$. Further, $\lim _{\mu \rightarrow 0} Q_{2}(t ; \mu)=0$, uniformly in every interval $0 \leqq t \leqq t_{0}<1$.

13. Compactness theorems. From the a priori estimates compactness theorems can be concluded. These theorems are tools for the existence proofs of the earlier paragraphs.

(i) Given a bounded sequence of solutions of the minimal surface equation in a domain $P$. There exists a subsequence whose functions, including their derivatives, converge to a solution of the minimal surface equation in $P$. The convergence is uniform in every compact subdomain of $P$.

(ii) Let $\left\{z_{n}(x, y)\right\}$ be a sequence of solutions of the minimal surface equation in $P$, converging to a continuous function $z(x, y)$ in $P$, uniformly in every compact subdomain of $P$. Then $z(x, y)$ is a solution of the minimal surface equation in $P$, and the derivatives of the $z_{n}(x, y)$ converge to the corresponding derivatives of $z(x, y)$, uniformly in every compact subdomain of $P$.

(iii) Given a monotonically increasing sequence $\left\{z_{n}(x, y)\right\}$ of solutions of the minimal surface equation in $P$. Assume that there is a function $M=M(x, y)<\infty$, defined in $P$ and independent of $n$, such that $z_{n}(x, y) \leqq M(x, y)$ for all $n$. Then the functions $z_{n}(x, y)$ and their derivatives converge to a solution of the minimal surface equation in $P$. The convergence is uniform in every compact subdomain of $P$.

Combining the last theorem and the situation described in II.11, 
one is led to the following statement due to H. Jenkins and J. Serrin ([1, p. 209]; see also R. Finn [6, p. 368]), which is used in dealing with the Dirichlet problem discussed in II.7.3:

(iv) Let $\left\{z_{n}(x, y)\right\}$ be a monotonically increasing sequence of solutions of the minimal surface equation in a bounded convex domain $P$. Denote by $V$ the empty or (by Harnack's inequality) open set of points in $P$ in which the limit function $z(x, y)$ is finite (and hence a solution of the minimal surface equation by (iii)). Each component of $V$ is bounded by straight line segments and subarcs of the boundary of $P$.

\section{Chapter III. Plateau's Problem}

1. Generalities about surfaces. For the following remarks see also the books by T. Radó [7] and L. Cesari [1].

1.1 A surface $S=\{T, P\}$ in Euclidean 3-space is a mapping $T$ given by a real nonconstant vector $\mathfrak{x}(u, v)=\{x(u, v), y(u, v), z(u, v)\}$, which is defined and continuous in a point set $P$ of the $(u, v)$-plane, the parameter domain (usually an open set bounded by finitely many Jordan curves), or, more generally, on an abstract differentiable surface $P$, the parameter surface (with or without boundary). Technically speaking, a surface $S$ has to be defined as a maximal class of Fréchet equivalent mappings, and each mapping $\{T, P\}$ of this class constitutes a particular representation of $S$.

By definition, the topological properties of the parameter domain determine the topological type of the surface, regardless of its appearance in space. Thus the surface $S_{1}$, represented by the mapping

$$
\left\{x=\cosh u \cos v, y=\cosh u \sin v, z=u ; u^{2}+v^{2}<\infty\right\},
$$

is simply connected, while the surface $S_{2}$, represented by the mapping

$$
\begin{aligned}
& \left\{x=\frac{1+u^{2}+v^{2}}{2\left(u^{2}+v^{2}\right)} u, y=\frac{1+u^{2}+v^{2}}{2\left(u^{2}+v^{2}\right)} v,\right. \\
& \left.\quad z=\frac{1}{2} \log \left(u^{2}+v^{2}\right) ; 0<u^{2}+v^{2}<\infty\right\},
\end{aligned}
$$

is doubly connected. Considered as point sets in space, both define the same surface, namely the catenoid $\left(x^{2}+y^{2}\right)^{1 / 2}=\cosh z$. The second mapping is globally one-to-one; the first mapping realizes an infinite covering.

We shall adopt the definition to call a surface to be genuinely of the topological type of its parameter domain $P$ if the mapping $T$ of $P$ into space realizes an imbedding, i.e., is one-to-one in the large.

If the parameter domain is open, we speak of an open surface (surface without boundary). If the parameter domain is bounded by 
$N$ distinct Jordan curves in the $(u, v)$-plane and if the vector $\mathfrak{x}(u, v)$ maps these curves monotonically onto $N$ distinct Jordan curves in space, then the surface is said to be bounded by the latter curves. It is then characterized by the triple of numbers $\mathfrak{I}(S)=[\epsilon, r, \chi]$, where $\epsilon= \pm 1$ describes the character of orientability, $r$ is the number of bounding contours, and $\chi$ denotes the Euler characteristic. It is customary to use catchwords, e.g., surface of the type of the disc $(\mathfrak{I}(S)=[1,1,1])$, surface of the type of the annulus $(\mathfrak{T}(S)=[1,2,0])$, surface of the type of the Moebius strip $(\mathfrak{T}(S)=[-1,1,0])$, etc.

A surface is called a saddle surface, if it has representation $\{\mathfrak{x}=\mathfrak{x}(u, v) ;(u, v) \in P\}$ for which all scalar products $\mathfrak{a} \cdot \mathfrak{x}(u, v)$ with constant vectors $\mathfrak{a}$ are Lebesgue-monotone functions in $P$.

1.2. Let $P$ be a bounded domain in the $(u, v)$-plane and $P_{1}$ a point set between the interior $P^{0}$ and the closure $\bar{P}$ of $P$. In the applications, $P$ will be an open connected set (such that $P^{0}=P$ ) and $P_{1}$ will be obtained from $P$ by including all boundary points (in the investigation of Plateau's problem) or some boundary points (in the investigation of free boundary value problems). A surface $S$ is said to belong to class $\mathfrak{B}\left(P_{1}\right)$ (reminding one of the notation in J. W. Calkin [1] and C. B. Morrey [2]), if it possesses a representation $\{\mathfrak{x}=\mathfrak{x}(u, v)$; $\left.(u, v) \in P_{1}\right\}$ in which the vector $\mathfrak{x}(u, v)$ is continuous in $P_{1}$, linearly absolutely continuous in $P^{0}$, and has square summable first derivatives in $P^{0}$. Then also the fundamental quantities $E=\mathfrak{x}_{u}^{2}, F=\mathfrak{x}_{u} \mathfrak{x}_{\mathfrak{v}}$, $G=\mathfrak{x}_{0}^{2}$ are summable in $P^{0}$, and the area $I(S)$ of such a surface is given by the classical integral

$$
I(S)=\iint_{P^{0}}\left(E G-F^{2}\right)^{1 / 2} d u d v .
$$

The above representation of $S \in \mathfrak{P}\left(P_{1}\right)$ is called almost conformal, if $E=G, F=0$ a.e. in $P^{0}$. If the vector $\mathfrak{x}(u, v)$ is continuously differentiable in $P^{0}$, these relations hold in all points of $P^{0}$. The representation is then termed conformal.

1.3. In general the mapping $T$ is no immersion, i.e., not even locally one-to-one, and surfaces, considered as point sets in space, may look rather bizarre. By imposing certain conditions, it can be enforced that a surface, at least locally, corresponds better to the intuitive picture one might have. (Self-intersections, self-contacts, coverings, and certain other unexpected peculiarities are still not excluded.)

$S$ is called a differential geometric surface, if it possesses a representation $\{\mathfrak{x}=\mathfrak{x}(u, v) ;(u, v) \in P\}$, whose vector belongs to class $C^{2}$ and satisfies the regularity condition $\mathfrak{x}_{u} \times \mathfrak{x}_{v} \neq 0$ in $P^{0}$. If, in addition, the mean curvature $H$ vanishes in all points of $P^{0}$, then the surface 
is called a minimal surface. We shall speak of a generalized differential geometric surface, or a generalized minimal surface-but this definition is only provisional-if the regularity condition is violated in isolated points of $P^{0}$.

2. The question of uniqueness. Plateau's problem, as it is generally understood, calls for a minimal surface of the type of the disc, bounded by a Jordan curve $\Gamma$ prescribed in space. ${ }^{5}$ The classical methods produce a generalized minimal surface in conformal representation as solution of Plateau's problem. If the curve $\Gamma$ is capable of spanning surfaces (of the type of the disc) of finite area-which, for instance, is the case for a rectifiable curve or a curve with a simply covered convex projection under parallel projection upon a planethen the above solution realizes the absolute minimum of the area for all surfaces of the type of the disc, spanned into $\Gamma$.

Of course, there may be more than one minimal surface, bounded by $\Gamma$. Examples of Jordan curves bounding at least two different minimal surfaces of the type of the disc-one of them, not realizing smallest area, explicitly known; the other one, realizing smallest area, known to exist but not explicitly available-have been given by T. Radó [5, pp. 2-8], [6, p. 40], and N. Wiener (see footnote on p. 269 in J. Douglas [2]). The example of a contour, bounding infinitely many minimal surfaces of the type of the disc, has been given, and related questions have been discussed by $\mathrm{R}$. Courant [3, pp. 119-122], and M. Kruskal [1]. Further examples have been suggested by $P$. Lévy [1], [2]. Applications of the theory of $M$. Morse by M. Morse and C. Tompkins, M. Shiffman, and R. Courant lead to deeper results.

However, only a few cases seem to be known in the literature of curves $\Gamma$, for which it can be stated that they bound exactly one, or definitely more than one (let alone a given number), minimal surfaces; and for the latter case not a single example seems to exist, in which all the minimal surfaces in question are explicitly known. (For

\footnotetext{
5Actually, Plateau himself formulates his "general principle" only in the following way (see [1, p. 213]): "Let there be given a surface of vanishing mean curvature. Imagine drawn on it a closed curve subject only to the conditions: (1) that it bound a finite portion of the surface, and (2) that this portion does not exceed the stability limit, in case the surface has such limits. Give the exact shape of the above-mentioned closed curve to a thin wire of iron, oxidize the latter a bit with diluted nitric acid, completely immerse it into the glycerine solution, and withdraw it. Then it will be filled by a lamina which represents the portion in question of the surface .... In this way, as by magic, one represents surfaces which, generally, are quite strange. The only difficulty consists in the selection of the closed curve and the exact determination of its shape. However, one will easily manage, if one knows either the equation or the geometrical generation of the surface."
} 
minimal surfaces of the type of the circular annulus the situation is more favorable: Two coaxial unit circles in not too distant parallel planes (see IV.1) as well as two interlocked unit circles in orthogonal planes bound two minimal surfaces of the type of the annulus.) $T$. Radó has proved uniqueness theorems under the assumption that the curve $\Gamma$ has a simply covered convex projection under central or parallel projection upon a plane.

\section{The isoperimetric inequality.}

3.1. As pointed out in III.2, the solution of Plateau's problem always appears in conformal representation, and it has been customary in many investigations to suppose any minimal surface under scrutiny to be given in a conformal representation. The theorems proved then quite properly could be considered as theorems on triples of conjugate harmonic functions.

To cite a notable example, most proofs of the isoperimetric inequality make this assumption. For such proofs, for minimal surfaces and more general surfaces, see T. Carleman [1], E. F. Beckenbach [1], E. F. Beckenbach and T. Radó [1], [2], R. Courant [3, pp. 129-131], A. Huber [1], [2], S. Lozinsky [1]. The simple closed character of the bounding contour is of no consequence for these proofs. T. Reid [1], and later C. C. Hsiung [1], published an interesting proof, which does not require the minimal surface under consideration to be given in a special representation. However, it requires continuous differentiability of the vector of the surface in the closure of the parameter domain.

On the strength of theorems due to E. J. McShane [1] and C. B. Morrey [1] (see also L. Cesari [1, p. 484])-and here the simple closed character of $\Gamma$, or, more generally, a nondegeneracy requirement, is essential - a minimal surface $S$ of the type of the circular disc, bounded by $\Gamma$, admits of a conformal representation, provided its area $I(S)$ is finite. Considering that there are Jordan curves which do not span any surface (of the type of the disc) of finite area, the question arises for conditions on the curve $\Gamma$ to ensure the finiteness of $I(S)$.

3.2. One would suspect that rectifiability of $\Gamma$ implies finiteness of $I(S)$. But it was only very recently that this conjecture was confirmed by C. Z. Šefel' [1]. ${ }^{6}$ His proof is quite complicated, and so far only a sketch of it has appeared. Sefel' shows that a saddle surface of the type of the disc, bounded by a rectifiable curve, has finite area and satisfies the isoperimetric inequality.

As we shall see below, the essential part of the proof consists in

6 Added in proof. Actually, this case can also be deduced from the investigations of E. F. Beckenbach and T. Rado [1]. 
establishing the isoperimetric inequality for saddle surfaces of class $\mathfrak{P}$ (of finite area). In his investigations, Šefel' considers the plane mappings defined by the three pairs of components of the vector of the surface.

Let $S=\left\{\mathfrak{x}=\mathfrak{x}_{0}(u, v) ;(u, v) \in \bar{P}\right\}$, where $\bar{P}$ denotes the closure of the unit disc $u^{2}+v^{2}<1$, be a minimal surface of the type of the disc, bounded by the Jordan curve $\Gamma$ of length $l$. Let $\epsilon_{n} \downarrow 0$ be a sequence of numbers and $\Sigma_{n}=\left\{\mathfrak{r}=\mathfrak{y}_{n}(u, v) ;(u, v) \in \bar{P}\right\}$ a sequence of polyhedra, bounded by simple closed curves $\Gamma_{n}$ of length $l_{n}$, such that $\left|l_{n}-l\right| \leqq \epsilon_{n}$ and $\left|\mathfrak{y}_{n}(u, v)-\mathfrak{x}_{0}(u, v)\right| \leqq \epsilon_{n}$ for $(u, v) \in \bar{P}$. For fixed $n$ pose the problem of finding a surface of smallest area among all $\mathfrak{P}(\bar{P})$-surfaces $\{\mathfrak{x}=\mathfrak{x}(u, v) ;(u, v)=\bar{P}\}$, bounded by $\Gamma_{n}$ and satisfying in $\bar{P}$ the inequalities

$$
\begin{aligned}
& \left|x(u, v)-x_{0}(u, v)\right| \leqq 2 \epsilon_{n}, \quad\left|y(u, v)-y_{0}(u, v)\right| \leqq 2 \epsilon_{n}, \\
& \left|z(u, v)-z_{0}(u, v)\right| \leqq 2 \epsilon_{n} .
\end{aligned}
$$

$\boldsymbol{\Sigma}_{n}$ itself is an admissible surface for this problem. Let $S_{n, m}$ be a minimizing sequence of admissible surfaces. The retractions $\hat{S}_{n, m}$, the vectors of which have Lebesgue-monotone components, and for which $I\left(\hat{S}_{n, m}\right) \leqq I\left(S_{n, m}\right)$, form also a minimizing sequence of admissible surfaces. By well-known theorems (see, for instance, E. J. McShane [1]) a subsequence of the $\hat{S}_{n, m}$ converges to a limit surface $\hat{S}_{n}$, a solution of the problem. The surface $T_{n}$, obtained from $\hat{S}_{n}$ by McShane's process of removal of excrescenses [2], is a saddle surface. Thus it is seen that $S$ can be approximated by saddle surfaces $T_{n} \in \mathfrak{P}(\bar{P})$ of finite area, bounded by the curves $\Gamma_{n}$.

3.3. It should be pointed out that Morrey's theorem, mentioned earlier in III.3.1, has been extended by W. H. Fleming [2] to nondegenerate surfaces of any finite topological type of finite area.

The question, whether a minimal surface or a saddle surface (of higher topological type), bounded by rectifiable contours, has finite area, seems never to have been discussed in the literature.

\section{Branch points.}

4.1. While the topics touched upon in the preceding two paragraphs are interesting and important, they do not affect the fundamentals and do not burden with doubt the reasonableness of Plateau's problem, as does the matter of branch points, or, more precisely, points in which the regularity condition is violated. (We worry only about branch points corresponding to interior points of the parameter domain.)

The behavior of generalized minimal surfaces in the neighborhood of a branch point and the complex relations, which exist between 
various types of branch points on certain generalized minimal surfaces, have been discussed by L. Bers [2], [3], Y. W. Chen [1], R. Courant $[3$, p. 123], and others. A generalized minimal surface $S$, given in a conformal representation can, after a rotation and a translation, locally be represented in the form

$$
\begin{aligned}
& x=\operatorname{Re}\left\{k w^{m}+a w^{m+1}+\cdots\right\}, \\
& y=\operatorname{Re}\left\{-i k w^{m}-i a w^{m+1}+\cdots\right\}, \\
& z=\operatorname{Re}\left\{b w^{m+n}+\cdots\right\} .
\end{aligned}
$$

Here $w=u+i v, k$ is a positive constant, $a$ and $b$ are complex constants, and the dots stand for terms with higher powers of $w$. The branch point on $S$ corresponds to the origin of the w-plane. The integers $m-1 \geqq 1$ and $n-1 \geqq 0$ are called, respectively, its order and index. In each sufficiently small neighborhood of the point $w=0$ there are at least two distinct points $w_{1} \neq 0, w_{2} \neq 0$, which are mapped into the same point in space by the vector of the surface. It does not follow from (34) that $S$ has $m$ different sheets in the neighborhood of the branch point. But Y. W. Chen proved the following statement:

If the numbers $m$ and $m+n$ are relatively prime, then the surface $S$ intersects itself in $(m-1)(m+n)$ (curved) rays, the branch lines, issuing from the branch point.

4.2. One would, without question, wish to obtain a minimal surface (and not only a generalized minimal surface) as solution of Plateau's problem. However, there are only a few types of contours $\Gamma$ for which it is known that the solution of the problem of least area or, for that matter, any generalized minimal surface, if it is given in a conformal representation, will not have branch points. By a theorem of T. Radó (see [4, p. 794], [6, p. 35]) this will be the case, provided there exists a straight line in space such that no plane through this line intersects the boundary curve in more than two distinct points. In particular, the assumptions of Rado's theorem are fulfilled for contours which possess a simply covered star-shaped Jordan curve as parallel or central projection upon some plane.

4.3. Recently S. Sasaki [1] gave a proof of W. Fenchel's theorem [1] about the total curvature of a closed space curve, employing the theory of minimal surfaces. His proof reversed (and corrected, see J. C. C. Nitsche [13]) leads to new information about minimal surfaces. Let $\Gamma$ be an analytic Jordan curve with arc length $s$ and curvature $k(s) \geqq 0$. Let $S=\{\mathfrak{x}=\mathfrak{x}(u, v) ;(u, v) \in \bar{P}\}$, where $\bar{P}$ is the closure of the unit circle $u^{2}+v^{2}<1$, be a generalized minimal surface of the type of the disc, bounded by $\Gamma$, given in a conformal representation. By a theorem of H. Lewy (see [5] and III.5.1) the vector of $\mathfrak{x}(u, v)$ 
is analytic in $\bar{P}$, and $S$ can have at most finitely many branch points. Let $\left(u_{\alpha}, v_{\alpha}\right) \quad(\alpha=1,2, \cdots, a)$ be the branch points of $S$ of orders $m_{\alpha}-1$, corresponding to points in $P$, and $\left(u_{\beta}, v_{\beta}\right)(\beta=1,2, \cdots, b)$ the branch points of $S$ of orders $M_{\beta}-1$, corresponding to points on $\partial P$. An application of the Gauss-Bonnet theorem then yields the inequality

$$
\int_{\Gamma} k(s) d s \geqq 2 \pi+\iint_{S}|K| d o+2 \pi \sum_{\alpha=1}^{a}\left(m_{\alpha}-1\right)+\pi \sum_{\beta=1}^{b}\left(M_{\beta}-1\right)
$$

and, in particular,

$$
1+\sum_{\alpha=1}^{a}\left(m_{\alpha}-1\right) \leqq \frac{1}{2 \pi} \int_{\Gamma} k(s) d s-\frac{1}{2 \pi} \iint_{S}|K| d o .
$$

Thus the surface $S$ cannot have an (interior) branch point if the total curvature of $\Gamma$ (which by Fenchel's theorem can never be smaller than $2 \pi$ ) is less than $4 \pi$. If the curve $\Gamma$ belongs to class $C^{2}$, then the same conclusion can be drawn at least for a minimal surface of smallest area bounded by $\Gamma$. Statements about any generalized minimal surface would, of course, be desirable, but seem to depend upon information unavailable at present concerning the boundary behavior of minimal surfaces (see III.5.2).

4.4. Formulas (35), (36) might lead one to speculate. If it were true that a generalized minimal surface of the type of the disc-and now it would be sufficient to talk of a surface of least area-bounded by a knotted curve, contained a branch point, then inequality (35) would lead to a new proof of J. Milnor's extension [1] of Fenchel's theorem. There are no topological reasons why a differential geometric surface of the type of the disc, bounded by a knotted curve, should have a branch point. So far the search for an example of a knotted Jordan curve $\Gamma$ with the property that any generalized minimal surface of the type of the disc, bounded by $\Gamma$ (or, at least, such a surface of smallest area), necessarily must have a branch point, has been without success.

As a matter of fact, it has sometimes been conjectured that a reasonable (analytic, differentiable, rectifiable, polygonal,... ?) curve $\Gamma$, knotted or not, always bounds a minimal surface (without branch points) of the type of the disc. The decision of this question seems to be one of the most important problems in the classical theory of Plateau's problem.

4.5. It is interesting to note that in R. Courant's example [3, p. 123] of a generalized minimal surface, bounded by a Jordan curve 


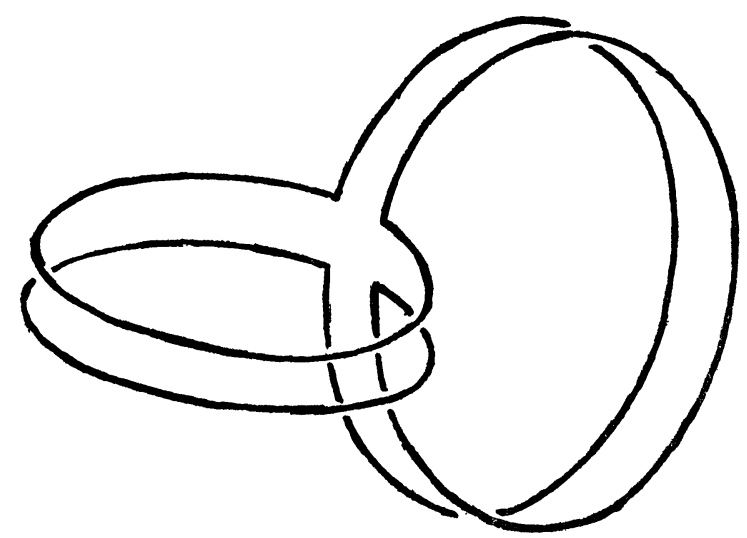

FIGURE 6

$\Gamma$, this curve is a knotted curve (an alternating torus knot). $\Gamma$ also bounds a minimal surface of the type of the Moebius strip and, as a matter of fact, it is the latter surface which generally comes out in soap film experiments.

This suggests that it might well be the insistence to obtain surfaces of least area of a prescribed, or at least restricted, topological type which is responsible for the occurrence of branch points. Various examples have been discussed in the literature of Jordan curves which bound minimal surfaces of different topological types. The best known of these, given by J. Douglas [5, p. 122], is sketched in Figure 6. We can consider this curve $\Gamma$ as boundary of the domain $D_{\delta}=\{\phi, \theta ;|\phi|<\delta\} \cup\{\phi, \theta ;|\theta|<\delta\}$ on the torus $\{x=(2-\cos \phi) \cos \theta, y=(2-\cos \phi) \sin \theta, z=\sin \phi ;|\phi| \leqq \pi,|\theta| \leqq \pi\}$, where $\delta$ is a small number $(0<\delta<1 / 10)$. Aside from bounding three different minimal surfaces of the type of the disc, $\Gamma$ will also bound a minimal surface of the type $[1,1,1]$ homeomorphic to $\bar{D}_{\delta}$. It can be shown that the area of a solution of Plateau's problem for $\Gamma$ is greater than $2 \pi(1-\delta)^{2}$, while the area of the domain $D_{\delta}$ is less than $16 \pi \delta$. Thus the minimal surface of type $[1,1,1]$ of smallest area, bounded by $\Gamma$, has an area which is smaller than that of any minimal surface of the type of the disc, bounded by $\Gamma$.

W. H. Fleming [1] has given a more sophisticated example of a rectifiable Jordan curve for which the problem of least area with unrestricted topological types has no solution of finite topological type, such that no surface of finite topological type realizes the least area among surfaces, bounded by $\Gamma$, with no restriction on the types.

\section{Boundary behavior.}

5.1. Let $S$ be a generalized minimal surface of the type of the disc, 
bounded by a rectifiable Jordan curve $\Gamma$ and given in a conformal representation $S=\{\mathfrak{x}=\mathfrak{x}(u, v) ;(u, v) \in \bar{P}\}$, where $\bar{P}$ is the closure of the unit disc $u^{2}+v^{2}<1$. The vector $\mathfrak{x}(u, v)$, for which we also write $\mathfrak{r}(w)$, is harmonic in $P$ and continuous in $\bar{P}$. Let $\mathfrak{x}^{*}(w)$ be the vector conjugate harmonic to $\mathfrak{x}(w)$. M. Tsuji [1] observed that the vectors $\mathfrak{x}\left(e^{i \theta}\right)$ and $\mathfrak{x}^{*}\left(e^{i \theta}\right)$ are absolutely continuous in $0 \leqq \theta \leqq 2 \pi$. He also proved an extension of the theorem of F. and M. Riesz [1], according to which a null set on $\partial P$ corresponds to a null set on $\Gamma$ and conversely, and the fact that the mapping of $\bar{P}$ onto $S$ is conformal in almost all points of $\partial P$. As a matter of fact, many theorems of function theory can be carried over to the present situation, i.e., to the theory of triples of conjugate harmonic functions. Investigations of this kind have been initiated by T. Radó and E. F. Beckenbach.

Assume now that $\Gamma$ contains an open analytic subarc $\Gamma_{0}$, corresponding to the arc $\gamma_{0}$ of $\partial P$. In this situation the following fundamental theorem, due to H. Lewy [5], holds:

The vector $\mathfrak{x}(u, v)$ is analytic in $P \cup \gamma_{0}$ and can be analytically continued across $\gamma_{0}$, i.e., the minimal surface $S$ can be analytically continued (as minimal surface) across the arc $\Gamma_{0}$.

An analysis of Lewy's proof shows that, once the surface is given in a conformal representation, the rectifiability of $\Gamma$ is immaterial for the proof. This proof makes essential use of the reflection principle for analytic functions and does not seem to carry over to the case where the $\operatorname{arc} \Gamma_{0}$ is merely sufficiently often differentiable, unless certain a priori estimates can be proved, estimates for derivatives of the vector $\mathfrak{x}(u, v)$ in terms of geometrical quantities of the arc $\Gamma_{0}$ and of finitely many derivatives of its vector.

5.2. So far the only estimate of this kind, which, however, is too weak for the above purposes, was obtained by S. E. Warschawski, combining the isoperimetric inequality with his method in conformal mapping [1]: Assume that there are an open arc $\Gamma_{0}$, containing the point $\mathfrak{x}\left(e^{i \theta 0}\right)$, of the rectifiable curve $\Gamma$ and a constant $c \geqq 1$ such that the distance $\Delta s$, measured along $\Gamma_{0}$, of any two points $\mathfrak{x}_{1}, \mathfrak{x}_{2}$ on $\Gamma_{0}$ satisfies the inequality $\Delta s \leqq c\left|\mathfrak{x}_{2}-\mathfrak{x}_{1}\right|$. Then we have

$$
\left|\mathfrak{x}(w)-\mathfrak{x}\left(e^{i \theta_{0}}\right)\right| \leqq C\left|w-e^{i \theta_{0}}\right| \alpha, \quad \alpha=\frac{2}{(1+c)^{2}} .
$$

For a continuously differentiable curve $\Gamma$ it follows from this result that the vector $\mathfrak{x}(u, v)$ belongs to class $C^{0, \beta}(\bar{P})$ for all $\beta<1 / 2$.

\section{Chapter IV. Doubly Connected Minimal Surfaces}

1. Experiments. Beautiful realizations of minimal surfaces can be obtained through soap films, spanned into a frame of thin wire con- 
sisting of one or several contours, by dipping this frame in a specially prepared soap solution and withdrawing it. Such experiments have been described in the classical works of J. Plateau [1], G. van der Mensbrugghe [1], C. V. Boys [1], H. A. Schwarz (see [1], Nachtrag on pp. 92-108) and others, and, more recently, among others, by G. A. Bliss (see [1], especially pp. 119-127), R. Courant [1], [4], and R. Courant and H. Robbins [1, pp. 385-397]. ${ }^{7}$

As anybody who has ever tried his hand at soap film experiments knows, however, it is not every time that a soap film develops in the frame. There can be two different reasons for this failure: Either a lack of skill on the part of the experimenter, or, more deeply seated, the mathematical fact that no minimal surface bounded by the curves of the frame exists at all. This point becomes already pertinent for frames consisting of two contours. In fact, while a Jordan curve in 3 -space always spans a (generalized) minimal surface of the type of the circular disc, the existence of a minimal surface of the type of the circular annulus, bounded by a system of two disjoint Jordan curves, depends very much on the configuration of these curves. A sufficient condition for the existence has been given by J. Douglas in famous papers [3], [7] and, in different versions, by $R$. Courant (see [3, especially Chapter IV]) and M. Shiffman [1], [2]. Roughly speaking, this criterion guarantees the existence of a minimal surface $S$ of the type of the circular annulus, bounded by two Jordan curves $\Gamma_{1}$ and $\Gamma_{2}$ without common points, provided these curves span a doublyconnected minimal surface whose area is smaller than the sum of the areas of the two simply-connected surfaces of least area spanned into $\Gamma_{1}$ and $\Gamma_{2}$, respectively.

Douglas's condition can be verified in many concrete cases. An interesting case is the one where the two curves $\Gamma_{1}$ and $\Gamma_{2}$ are interlocked. Nevertheless, even if Douglas's condition is violated, a minimal surface of the type of the annulus, bounded by $\Gamma_{1}$ and $\Gamma_{2}$, may still exist. This fact is well illustrated with the classical experiment where the curves $\Gamma_{1}$ and $\Gamma_{2}$ are coaxial unit circles in parallel planes, say the planes $z=-h$ and $z=h(h>0)$; see for instance the presentation in G. A. Bliss [1]. For $h<h_{1}=0.5277 \ldots$ Douglas's condition is fulfilled. For $h_{1} \leqq h \leqq h_{2}=0.6627 \ldots$ Douglas's condition is violated; yet the circles $\Gamma_{1}$ and $\Gamma_{2}$ still span a doubly-connected minimal

${ }^{7} \mathrm{G}$. van der Mensbrugghe has this to say about the experiments of Plateau (see [2, p. 433]): "There is, to our knowledge, no example where the observation would have supported the theory with more ravishing forms. What could appear more beautiful to the eyes of a mathematician than these weightless models, adorned with the most brilliant colors, and, despite their extraordinary fragility, of an astonishing persistence." 
surface, namely a catenoid (for $h<h_{2}$ even two catenoids). It is not before $h$ exceeds the value $h_{2}$ that there does no longer exist a minimal surface of the type of the circular annulus bounded by $\Gamma_{1}$ and $\Gamma_{2}$. All this can be discussed by means of simple transcendental equations. Actually, these equations tell us only that no catenoid exists. It is a fact, however, that no minimal surface of the type of the annulus exists at all (see IV.3). If we, contrariwise, keep the distance $2 h$ fixed, but move the curves $\Gamma_{1}$ and $\Gamma_{2}$ sideways, we will again obtain a limit value $d=d_{2}(h)$ of their lateral distance $d$, such that no minimal surface of the type of the circular annulus bounded by $\Gamma_{1}$ and $\Gamma_{2}$ exists for $d>d_{2}$. The determination of this value $d_{2}(h)$ is possible, though considerably more complicated, and has never been carried out.

2. Necessary criteria for existence. Speaking generally again it seems clear that the curves $\Gamma_{1}$ and $\Gamma_{2}$ will not span a doubly-connected minimal surface, if they are too far apart. Quantitative criteria making this suggestion precise have never been given in the literature, however. It may be of interest, therefore, to see such a criterion.

Let us say that the Jordan curves $\Gamma_{1}$ and $\Gamma_{2}$ are separated by a slab of width $r>0$, if there are two parallel planes of distance $r$, such that the curves $\Gamma_{1}$ and $\Gamma_{2}$ do not enter the domain between the planes and are separated by each of the planes. The following theorem holds (see J. C. C. Nitsche [17]):

Given two Jordan curves $\Gamma_{1}$ and $\Gamma_{2}$ of diameters $d_{1}$ and $d_{2}$, respectively, which are separated by a slab of width $r>0$. If these curves bound a minimal surface of the type of the circular annulus, then the inequality

$$
r \leqq \frac{3}{2} \operatorname{Max}\left(d_{1}, d_{2}\right)
$$

must be satisfied.

This inequality tells us how far, at best, the curves $\Gamma_{1}$ and $\Gamma_{2}$ can be apart in order still to span a doubly-connected minimal surface; and in this sense it may be considered as a supplement to Douglas's condition. The inequality (38) is not sharp. If the curves $\Gamma_{1}$ and $\Gamma_{2}$ lie in parallel planes, say the planes $z=c_{1}$ and $z=c_{2}\left(c_{1}<c_{2}\right)$, respectively, a somewhat better result, which, however, is not sharp either, can be obtained; see J. C. C. Nitsche [15]:

Let us choose a point $p_{1}=\left(x_{1}, y_{1}, c_{1}\right)$ in the plane $z=c_{1}$, in some sense the center of $\Gamma_{1}$, and a point $p_{2}=\left(x_{2}, y_{2}, c_{2}\right)$ in the plane $z=c_{2}$, in some sense the center of $\Gamma_{2}$. The distance between the two curves $\Gamma_{1}$ and $\Gamma_{2}$ can then be measured in terms of the two quantities $r=c_{2}-c_{1}$ (vertical distance) and $d=\left[\left(x_{2}-x_{1}\right)^{2}+\left(y_{2}-y_{1}\right)^{2}\right]^{1 / 2}$ (lateral distance). We further denote by $\delta_{1}$ the maximal distance of the point $p_{1}$ from the 
curve $\Gamma_{1}$ and by $\delta_{2}$ the maximal distance of the point $p_{2}$ from the curve $\Gamma_{2}$. We can then state:

If the curves $\Gamma_{1}$ and $\Gamma_{2}$ bound a minimal surface of the type of the circular annulus, then the distance between these curves must satisfy the inequality

$$
\left(r^{2}+\frac{1}{2} d^{2}\right)^{1 / 2} \leqq \delta_{1}+\delta_{2} .
$$

3. The theorems of M. Shiffman. For such surfaces, i.e., minimal surfaces $S$ bounded by curves $\Gamma_{1}$ and $\Gamma_{2}$ in two parallel planes $z=c_{1}$ and $z=c_{2}$, interesting facts have been discovered by M. Shiffman [3]. He proved that if the two curves $\Gamma_{1}$ and $\Gamma_{2}$ are convex, then also all curves $S_{c}$ of intersection of $S$ with the planes $z=c\left(c_{1}<c<c_{2}\right)$ must be convex. Using the Sturm-Liouville theory applied to a differential equation, which also plays a role in the investigation of the second variation of the area, Shiffman also proves the deeper theorem that all the curves $S_{c}\left(c_{1}<c<c_{2}\right)$ must be circles, if the curves $\Gamma_{1}$ and $\Gamma_{2}$ are circles. Shiffman's proof assumes that the minimal surface $S$ under consideration is already given in a conformal representation. If this is not the case it seems that certain regularity assumptions have to be made for Shiffman's theorems to be valid.

Minimal surfaces which are generated by a family of circles in parallel planes had been determined already in the last century by A. Enneper [2] and B. Riemann [1], who obtained their representation in terms of elliptic integrals. In fact, Enneper proved that if a minimal surface is generated by a one-parameter family of circles, then these circles must lie in parallel planes. From the explicit representation it follows, in particular, that the minimal surface must be a catenoid, if the circles $\Gamma_{1}$ and $\Gamma_{2}$ are coaxial. It is only the recent result of M. Shiffman, which tells us now-to be sure, under slight regularity assumptions for the vector of the surface on the boundary - that any minimal surface of the type of the circular annulus, bounded by two coaxial circles, must be a catenoid. (For a surface of least area this follows, of course, by a symmetrization argument.)

Minimal surfaces, which are bounded by two coaxial regular $n$ polygons in parallel planes, and their explicit representation, have, under certain symmetry assumptions, been discussed by $H$. A. Schwarz [4]. He considers in particular the case $n=4$. The case of the catenoid is obtained for $n \rightarrow \infty$.

4. Surfaces of class $\mathfrak{S}$. Aside from the minimal surfaces which are generated by a family of circles in parallel planes, the minimal surfaces with a representation in cylindrical coordinates fall under the category of surfaces considered here. For the latter surfaces, each 
curve $S_{c}$ is a star-shaped curve in the plane $z=c$ (star-shaped with respect to the point $x=y=0$ ).

Let us more generally consider minimal surfaces $S$-and now we are talking of open surfaces-with the property that each curve $S_{c}$ is star-shaped with respect to an interior point, depending on $c$. Such a surface, defined in the slab $|z|<r$, is called of class $\subseteq(r)$.

A few years ago I had proved in [10] (see also [3], [9]) that a minimal surface of class $\mathfrak{S}(\infty)$-or, more generally, a complete minimal surface of class $\mathfrak{S}(r)$-must be a catenoid. This means that a doubly connected minimal surface of class $\subseteq(r)$ cannot be continued to infinity, or to a complete surface, in both $z$-directions in such a way that all curves $S_{c}$ remain star-shaped, unless the original portion of the surface is part of a catenoid.

This result reminds one of Bernstein's theorem on minimal surfaces with a representation $z=z(x, y)$. As we have seen in II.9.3 and II.9.4, quantitative proofs of this theorem construct a positive function $M(R)$, tending to zero as $R \rightarrow \infty$, with the following property: If a minimal surface $z=z(x, y)$ is defined over the disc $\left(x-x_{0}\right)^{2}+\left(y-y_{0}\right)^{2}$ $<R^{2}$, then its Gaussian curvature must satisfy the inequality $\left|K\left(x_{0}, y_{0}\right)\right| \leqq M(R)$. Identically vanishing Gaussian curvature, of course, is characteristic for a plane.

The question arises whether there is a quantitative analogue to Bernstein's theorem for the case of doubly-connected minimal surfaces of the type under discussion. Then, of course, it is first necessary to find a suitable property characterizing catenoids. It turns out that the deviation from the circular shape of any individual curve $S_{c}$, expressed in terms of its length $L$ and the quotient $k_{1} / k_{2}$ of its extreme curvatures, leads to a useful measure. Roughly speaking, the result will be the following: A minimal surface $S$, which contains a noncircular curve $S_{c_{0}}$, cannot be continued to infinity in both $z$-directions in such a way that all curves $S_{c}$ remain star-shaped. And more: It depends on the deviation of the curve $S_{c_{0}}$ from the circular shape, how far the continuation can be carried. It may be possible to continue $S$ in the described way to infinity in one $z$-direction, but not in both.

It is my conjecture that similar results hold for surfaces of class $\Im(r)$, i.e., surfaces defined in the slab $|z|<r$ for which all the curves $S_{c}(|c|<r)$ are Jordan curves, in particular, that a minimal surface of class $\Im(\infty)$, i.e., a minimal surface which is intersected by all planes $z=$ const in Jordan curves, or, more generally, a complete surface of class $\Im(r)$, must be a catenoid. But so far all attempts to prove (or disprove) this conjecture have been unsuccessful. 
1965] ON NEW RESULTS IN THE THEORY OF MINIMAL SURFACES
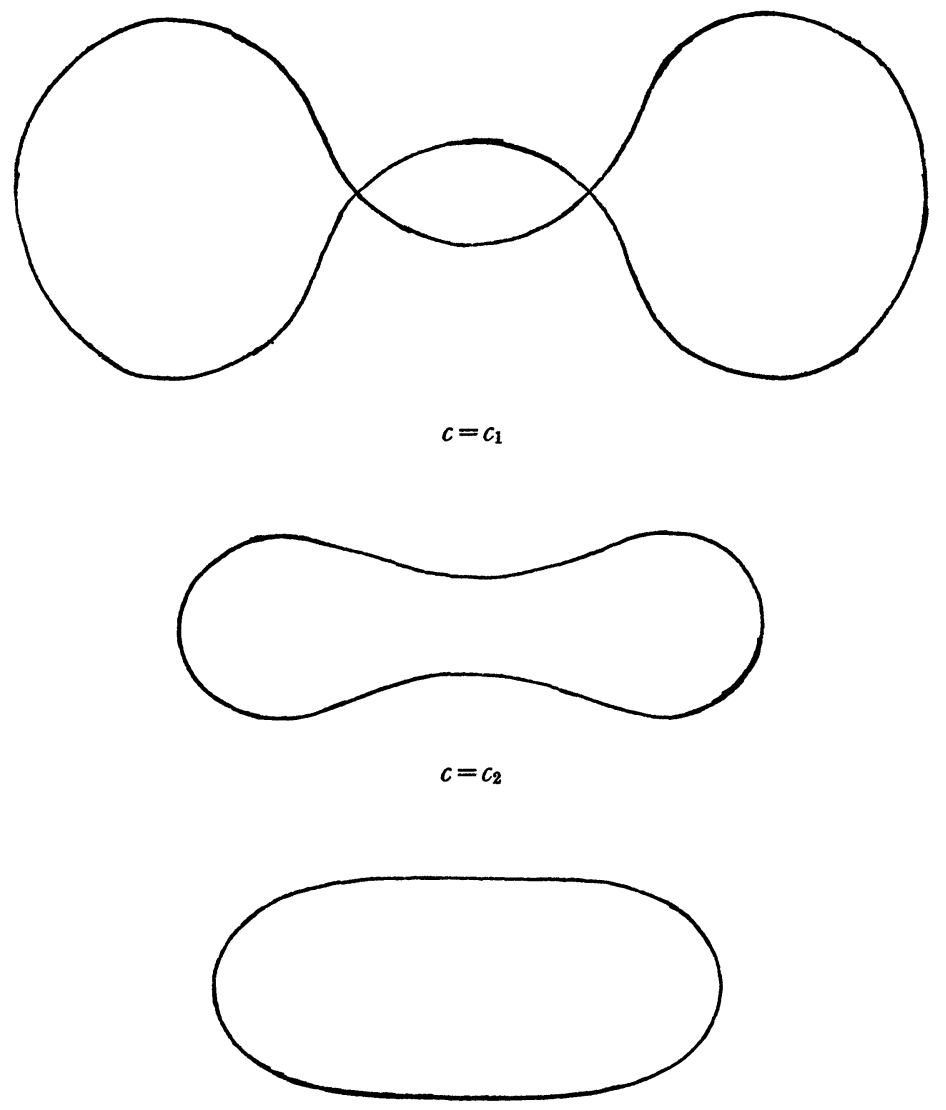

$c=c_{3}$

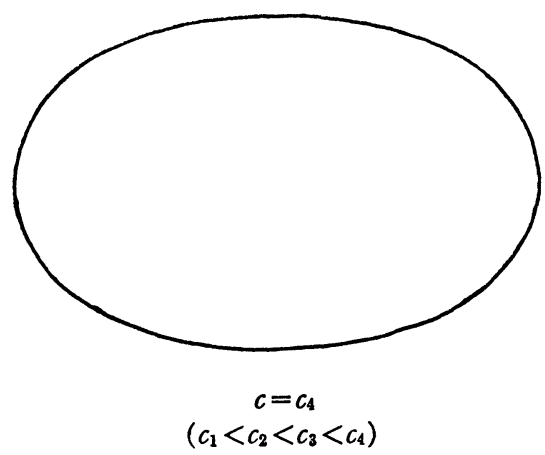

Figure 7 
Figure 7 (taken from J. C. C. Nitsche [10]) gives an illustration of the general situation. It shows for a special minimal surface the curves $S_{c}$ in planes of various heights. One sees that, proceeding from negative values of $c$ to positive values of $c$, the curves $S_{c}$ are convex and almost circles at first. At a certain point they cease being convex but still stay star-shaped. Later on they lose this property too, remaining Jordan curves for a while, until finally they begin intersecting themselves, getting wilder and wilder for large values of $c$.

Precisely, it is the following theorem which has been proved (see J. C. C. Nitsche [12]):

Let $S$ be a minimal surface of class $\subseteq(r)$. Denote by $k_{1}$ and $k_{2}$ the (non-negative) minimum and maximum curvatures of the curve $S_{0}$ (intersection of $S$ with the plane $z=0$ ) and by $L$ its length. There are a constant $q_{0}>0$ and a function $M(q)$, defined for $q \geqq q_{0}$ and satisfying $0<M(q)<1$ and $M(q) \rightarrow 1$ for $q \rightarrow \infty$ such that

$$
k_{1} / k_{2} \geqq M(r / L) ;
$$

i.e., for fixed $L$ : The larger $r$ the closer to one must be the quotient $k_{1} / k_{2}$.

This theorem allows us to estimate explicitly how far, at best, a minimal surface, containing a noncircular arc $S_{c_{0}}$, can be extended in both $z$-directions in such a way that all curves $S_{c}$ remain star-shaped.

5. The theorems of $\mathrm{K}$. Leichtweiss. While catenoids are the prototypes of the minimal surfaces considered in the preceding paragraphs, the helicoids are examples of surfaces for which all level curves $S_{c}$ are open curves of infinite length. Inspired by the theorem that a surface of class $\mathfrak{S}(\infty)$ must be a catenoid, K. Leichtweiss (in yet unpublished work) proved two theorems characterizing helicoids.

(i) Let $S$ be a complete minimal surface satisfying the following assumptions:

(1i) $S$ has a representation

$$
\left\{x=f(z, s), y=g(z, s) ; c_{1}<z<c_{2},-\infty<s<\infty\right\}
$$

where $-\infty \leqq c_{1}<c_{2} \leqq \infty$, and $f$ and $g$ have continuous second derivatives. $s$ is the arc length on the level lines $S_{c}=\{x=f(c, s), y=g(c, s) ;-\infty<s$ $<\infty\}$, and the curve $\left\{x=f(z, 0), y=g(z, 0) ; c_{1}<z<c_{2}\right\}$ is an orthogonal trajectory of the level lines $S_{c}$.

(2i) The level lines $S_{d_{1}}$ and $S_{d_{2}}\left(c_{1}<d_{1}<d_{2}<c_{2}\right)$ are straight lines of distance $d_{2}-d_{1}$.

(3i) The unit normal vector $\mathfrak{X}(z, s)$ of $S$ satisfies, uniformly for all $z$ in $d_{1} \leqq z \leqq d_{2}$, the relations

$$
\lim _{s \rightarrow-\infty} \mathfrak{X}(z, s)=-\lim _{z \rightarrow+\infty} \mathfrak{X}(z, s)=(0,0,1) .
$$


Then $S$ is a right helicoid whose axis is parallel to the z-axis.

(ii) Let $S$ be a complete minimal surface satisfying the following assumptions:

(1ii) Assumption (1i) of the previous theorem.

(2ii) For each $s_{0}>0$ the Gaussian curvature $K(z, s)$ of $S$ satisfies Sup $K(z, s)<0$, where the supremum is taken over all $c_{1}<z<c_{2},|s| \leqq s_{0}$.

(3ii) (41) is satisfied uniformly for $z$ in $c_{1}<z<c_{2}$.

Then $S$ is a right helicoid whose axis is parallel to the z-axis.

\section{Chapter V. Complete Minimal Surfaces}

\section{Generalizations of Bernstein's theorem.}

1.1. The proof of Bernstein's theorem given in II.9.1 uses certain properties of the function $\Omega(\zeta)$. This function has an obvious geometrical interpretation: A point in the $\zeta$-plane defines a point on the surface. The latter point is mapped by parallel normals onto the unit sphere. Mapping this spherical image by stereographic projection from the north pole into the equator plane the function $\overline{\Omega(\zeta)}$ results.

If one looks for the reasons why the proof of II.9.1 works, one will find it in the completeness of the minimal surface-from which by the use of the conformal mapping (8) and its property (i) the conformal parabolic character of the minimal surface follows-and in the boundedness of the analytic function $\Omega(\zeta)$-from which it follows that the spherical image of the minimal surface is contained in a semi-sphere.

L. Nirenberg asked whether a theorem similar to Bernstein's theorem holds for minimal surfaces which do not necessarily admit a nonparametric representation. In answer, $R$. Osserman [1] proved the theorem:

A complete minimal surface, whose normals omit the neighborhood of some direction, must be a plane.

1.2. We shall sketch here the proof for a simply connected minimal surface. (In the general case the universal covering of our minimal surface has to be considered.) The surface can be given in its Weierstrass representation

$$
\begin{aligned}
& x=x_{0}+\operatorname{Re} \int_{0}^{\zeta}\left(\Phi^{2}-\Psi^{2}\right) d \xi^{\prime} \\
& y=y_{0}+\operatorname{Re} \int_{0}^{\zeta} i\left(\Phi^{2}+\Psi^{2}\right) d \xi^{\prime} \\
& z=z_{0}+\operatorname{Re} \int_{0}^{\zeta} 2 \Phi \Psi d \zeta^{\prime}
\end{aligned}
$$


where $\zeta$ ranges over the normal domain $P_{0}$-the unit circle $|\zeta|<1$ (hyperbolic case) or the finite $\zeta$-plane (parabolic case) - and where $\Phi(\zeta)$ and $\Psi(\zeta)$ are single-valued analytic functions without common zeros in $P_{0}$. We remark that every minimal surface of the type of the open disc has such a representation (42) and, conversely, that every pair of functions $\Phi(\zeta), \Psi(\zeta)$ with the above mentioned properties, substituted into (42), leads to a minimal surface of the type of the open disc. The function $\omega(\zeta)=\Psi(\zeta) / \Phi(\zeta)$ is realized, as was before the function $\overline{\Omega(\zeta)}$, by the mapping of the minimal surface into the equator plane of the spherical image. We may assume that $(0,0,1)$ is the direction whose neighborhood is omitted by the spherical image. Then the function $\Phi(\zeta)$ cannot vanish, and $\omega(\zeta)$ is bounded: $|\omega(\zeta)| \leqq m$.

We shall now first prove that $P_{0}$ cannot be the unit circle, i.e., that the surface is of conformal parabolic type. To this end the following function-theoretic lemma, due to R. Osserman [5, p. 71], is used:

Let $f(\zeta)$ be analytic and $f(\zeta) \neq 0$ in $|\zeta|<1$. There exists a path $\gamma$ going from $\zeta=0$ to the boundary of $|\zeta|<1$, such that

$$
\int_{\gamma}|f(\zeta)||d \zeta|<\infty
$$

(In fact, $\int_{\gamma}|f(\zeta)||d \zeta| \leqq|f(0)|$.) Theorems of this type, in a considerably more general setting, were first proved and used by A. Huber (see [3, especially Theorem 2, p. 20]).

The arc length of a curve on the minimal surface is given by the relation $d s=\left(|\Phi|^{2}+|\Psi|^{2}\right)|d \zeta| \leqq\left(1+m^{2}\right)|\Phi(\zeta)|^{2}|d \zeta|$. Letting $c$ be the image path of $\gamma$ on the minimal surface and identifying the function $\Phi^{2}(\zeta)$ with the function $f(\zeta)$ of the lemma, we find for the length $L$ of $c$, the inequality

$$
L=\int_{c} d s=\int_{\gamma}\left(|\Phi|^{2}+|\Psi|^{2}\right)|d \zeta| \leqq\left(1+m^{2}\right) \int_{\gamma}\left|\Phi^{2}(\zeta)\right||d \zeta|<\infty .
$$

Considering that the path $c$ leads to the boundary of the surface and that the surface is assumed to be complete, a contradiction is obtained.

Once we know that the normal domain $P_{0}$ is the whole plane, the application of Liouville's theorem to the function $\omega(\zeta)$ again shows that this function must be a constant, and again it follows that the minimal surface must be a plane.

1.3. Let us note that the variable $\omega$ can be used in (42) in the neighborhood of a nonumbilical point in which the function $\phi$ does 
not vanish (an assumption which can be made without loss of generality). Then the classical representation of $\mathrm{K}$. Weierstrass,

$$
\begin{aligned}
& x=x_{0}+\operatorname{Re} \int_{\omega_{0}}^{\omega}\left(1-\omega^{\prime 2}\right) g\left(\omega^{\prime}\right) d \omega^{\prime} \\
& y=y_{0}+\operatorname{Re} \int_{\omega_{0}}^{\omega} i\left(1+\omega^{\prime 2}\right) g\left(\omega^{\prime}\right) d \omega^{\prime} \\
& z=z_{0}+\operatorname{Re} \int_{\omega_{0}}^{\omega} 2 \omega^{\prime} g\left(\omega^{\prime}\right) d \omega^{\prime}
\end{aligned}
$$

is obtained (see, for instance, K. Weierstrass [1], L. Bianchi [1, p. $360]$, T. Radó [6, p. 38]). Each minimal surface is thus characterized by an analytic function.

To cite a few well-known examples: The functions $g(\omega)=1$, $g(\omega)=-1 / 2 \omega^{2}, g(\omega)=i / 2 \omega^{2}, g(\omega)=2 /\left(1-\omega^{4}\right), g(\omega)=1-1 / \omega^{4}, g(\omega)$ $=\left(1-14 \omega^{4}+\omega^{8}\right)^{-1 / 2}$ lead, respectively, to A. Enneper's surface (example (ii) in II.2.3), catenoid, helicoid, H. F. Scherk's surface (6'), L. Henneberg's surface [1], [2], and the surface of H. A. Schwarz and B. Riemann (see II.2.4).

How (43) can be made a global representation is illustrated in V.1.5.

1.4. In a similar fashion as for Bernstein's theorem of II.9.3 a quantitative version of the preceding theorem can be given by proving an estimate for the Gaussian curvature (see R. Osserman [3], [4] and the remark on p. 75 of [5]):

Let $p$ be a point of a minimal surface and $N$ a neighborhood of $p$. Let the distance along the surface from $p$ to the boundary of $N$ be at least $d$. Assume that for some $\alpha>0$ all normals to points of $N$ form an angle of at least $\alpha$ with some fixed direction, and let the angle between this fixed direction and the normal at $p$ be $\beta$. Then the Gaussian curvature $K_{p}$ at $p$ satisfies the inequality

$$
\left|K_{p}\right| \leqq c(\alpha, \beta) d^{-2} \text {. }
$$

Here $c(\alpha, \beta)$ is an explicitly given constant, depending on $\alpha$ and $\beta$.

1.5. Considering that Liouville's theorem plays a prominent role in the argument of V.1.2, one might suspect that there will also exist an analogue to Picard's theorem. There are, however, examples of complete minimal surfaces, different from a plane, whose normals omit three or four directions.

The surface of H. A. Schwarz and B. Riemann (see II.2.4), continued across its sides by repeated reflection, is a complete minimal 
surface whose normals assume every direction; A. Enneper's surface (example (ii) in II.2.3) has a spherical image omitting one point; the spherical image of the catenoid and the spherical image of the (complete) right helicoid $\{x=u \cos v, y=u \sin v, z=v ;|u|,|v|<\infty\}$ omit two points; the spherical image of H. F. Scherk's surface (6)i.e., the complete infinitely connected surface (see below)-omits four points, namely $\omega= \pm i e^{ \pm i \alpha}$. (For the surface $\left(6^{\prime}\right)$ the points omitted are $\omega= \pm 1, \pm i$.) Other examples with three or four omitted points were given by R. Osserman in [5, p. 75 and p. 72]. K. Voss (according to a communication by $\mathrm{R}$. Osserman) noted that one can prescribe arbitrarily four points on the sphere and find a complete minimal surface whose spherical image omits exactly these points.

One can determine such a simply connected minimal surface easily as follows: Let $\omega_{1}, \omega_{2}, \omega_{3}, \omega_{4}$ be four given points in the $\omega$-plane. In view of the possibility of a preliminary rotation of the surface to be determined, it is no loss of generality to assume that $\omega_{k} \neq \infty$ $(k=1,2,3,4)$. Denote by $R$ the extended $\omega$-plane, punctured at the points $\omega_{k}$, and by $\hat{R}$ the universal covering surface of $R$. Let $\omega=\hat{\omega}(\zeta)$ be a one-to-one conformal mapping of the unit disc $P_{0}=\{\zeta ;|\zeta|<1\}$ onto $\hat{R}$ and $\tilde{\omega}$ the projection map of $\hat{R}$ onto $R$. Then the mapping $\omega=\omega_{0}(\zeta) \equiv \tilde{\omega}(\hat{\omega}(\zeta))$ is locally one-to-one, and we have $\omega_{0}^{\prime}(\zeta) \neq 0$ in $P_{0}$. We also may assume that $\omega_{0}(0) \neq \infty$.

Now set $g(\omega)=\left[\left(\omega-\omega_{1}\right)\left(\omega-\omega_{2}\right)\left(\omega-\omega_{3}\right)\left(\omega-\omega_{4}\right)\right]^{-1}$. Then the surface

$$
\begin{aligned}
& x=\operatorname{Re} \int_{0}^{\zeta}\left(1-\omega_{0}^{2}\left(\zeta^{\prime}\right)\right) g\left(\omega_{0}\left(\zeta^{\prime}\right)\right) \omega_{0}^{\prime}\left(\zeta^{\prime}\right) d \zeta^{\prime} \\
& y=\operatorname{Re} \int_{0}^{\zeta} i\left(1+\omega_{0}^{2}\left(\zeta^{\prime}\right)\right) g\left(\omega_{0}\left(\zeta^{\prime}\right)\right) \omega_{0}^{\prime}\left(\zeta^{\prime}\right) d \zeta^{\prime} \quad\left(\zeta \in P_{0}\right) \\
& \left.z=\operatorname{Re} \int_{0}^{\zeta} 2 \omega_{0}\left(\zeta^{\prime}\right) g\left(\omega_{0}\left(\zeta^{\prime}\right)\right) \omega_{0}^{\prime}\left(\zeta^{\prime}\right)\right) d \zeta^{\prime}
\end{aligned}
$$

is a minimal surface $S$ with the desired properties, as we shall show presently. (Note the relation of (45) to (43)!)

A path $\lambda$ in $P_{0}$ defines a curve on $S$. Its line element is given by

$$
\begin{aligned}
d s & =\left(1+\left|\omega_{0}(\zeta)\right|^{2}\right)\left|g\left(\omega_{0}(\zeta)\right)\right|\left|\omega_{0}^{\prime}(\zeta)\right||d \zeta| \\
& =\left(1+|\omega|^{2}\right)|g(\omega)||d \omega|,
\end{aligned}
$$

where the last expression is defined on the image path $\lambda^{\prime}$ of $\lambda$ in the $\omega$-plane. It is clear from this that the points in $P_{0}$, which are mapped onto $\omega=\infty$, define regular points on $S$. It is also clear that the spherical image of $S$ omits precisely the points $\omega_{k}$. As for the completeness, we proceed as follows. 
Let $\lambda$ be a half-open path in $P_{0}$, leading from the point $\zeta=0$ to the boundary of $P_{0}$, and $\lambda^{\prime}$ its image in the $\omega$-plane under the mapping $\omega=\omega_{0}(\zeta)$. Three cases are possible:

1. $\lambda^{\prime}$ has finite length with respect to the Euclidean metric in the $\omega$-plane. Then $\lambda^{\prime}$ must tend to a definite point $\omega^{\prime} \neq \infty$. Considering that $\lambda$ leads to the boundary of $P_{0}$, one sees that $\omega^{\prime}$ must be one of the points $\omega_{k}$.

2. $\lambda^{\prime}$ has infinite length and stays in a compact subdomain of the (finite) $\omega$-plane.

3. $\lambda^{\prime}$ has infinite length, but does not stay in any compact subdomain of the (finite) $\omega$-plane. Considering that $\lambda$ leads to the boundary of $P_{0}, \lambda^{\prime}$ cannot converge to $\omega=\infty$.

The length $L$ of the curve on $S$, defined by $\lambda$, is equal to

$$
L=\int_{\lambda} d s=\int_{\lambda^{\prime}}\left(1+|\omega|^{2}\right)|g(\omega)||d \omega| .
$$

It is easy to prove that $L=\infty$ in each of the three cases. Thus the minimal surface $S$ is complete.

It is not claimed that $S$ is genuinely simply connected (see III.1.1). If there is a subcovering of $R$ on which the coordinates of the surface are already single valued, then a surface of genuinely higher connectivity is obtained. This situation is illustrated by H. F. Scherk's surface $\left(6^{\prime}\right)$, where $g(\omega)=2 /\left(1-\omega^{4}\right)$, so that

$$
\begin{aligned}
& x=\operatorname{Re}\left\{\frac{1}{i} \log \frac{i-\omega}{i+\omega}\right\} \\
& y=\operatorname{Re}\left\{i \log \frac{1+\omega}{1-\omega}\right\} \\
& z=\operatorname{Re}\left\{\log \frac{1+\omega^{2}}{1-\omega^{2}}\right\} .
\end{aligned}
$$

If we take as parameter domain the minimal covering surface $R_{0}$ of the extended $\omega$-plane punctured at $\omega= \pm 1, \pm i$, on which the functions $\left(46_{1,2}\right)$ are single valued, it has been shown by $R$. Osserman (see $[8$, pp. 11-13]) that Scherk's surface is genuinely infinitely connected.

One might call the surfaces (45), whose explicit representation can easily be written down, general Scherk surfaces. To our knowledge, their geometrical properties (do they contain straight lines or interesting families of curves as Scherk's surface does, etc.?) have never been studied. 
It has been conjectured that the number four entering the preceding constructions might characterize a critical limit; see R. Osserman $[8$, p. 15 and problem 47 in Chapter VII].

Of course, the normal map of the minimal surface upon its spherical image will in general not be one-to-one. It has been shown independently by R. Osserman $[8$, p. 16] and K. Voss that A. Enneper's surface and the catenoid are the only surfaces whose normal map is one-to-one.

1.6. Conditions concerning a special representation (as in II.9.1) or properties of the spherical image (as in V.1.1) are convenient means of forcing a complete minimal surface to be a plane.

It would be interesting to find other geometrical properties with the same effect. For instance, one might think that the requirement that the minimal surface should be imbedded in space, i.e., the mapping $T$ of $P$ should be globally one-to-one, could be of this kind. The minimal surface of infinite genus, determined by E. R. Neovius (see [1], where a beautiful photograph of this surface can be found in Table IV; see also D. Hilbert and S. Cohn-Vossen [1, p. 271]), as well as the helicoid, however, frustrate this expectation.

2. Two lemmas. Of the various results in the spirit of the lemma stated in V.1.2, which are useful for the study of minimal surfaces, we mention the following two:

(i) Let the function $f(\zeta)$ be analytic and $f(\zeta) \neq 0$ in $0<r_{1}<|\zeta|<r_{2}$ $<\infty$. There exists a path $\gamma$ leading from the point $\zeta_{0}=\left[\mu r_{1} r_{2}\right]^{1 / 2}$ (with $\left.r_{1}<\left|\zeta_{0}\right|<r_{2}\right)$ to the outer boundary $|\zeta|=r_{2}$, such that

$$
\int_{\gamma}|f(\zeta)||d \zeta| \leqq M\left|f\left(\zeta_{0}\right)\right|^{2}
$$

Here $\mu$ is an arbitrary number $\left(1<\mu<r_{2} / r_{1}\right)$, and

$$
M=\frac{2}{\pi} \log \left(\frac{r_{2}}{\mu r_{1}}\right)\left[\operatorname{Min}_{\mu r_{1} \leq|\zeta| \leq\left(\mu r_{12} r^{1 / 2}\right.}|f(\zeta)|\right]^{-1} .
$$

(ii) Let the function $f(\zeta)$ be analytic and $f(\zeta) \neq 0$ in $0<|\zeta|<1$. There exists a path $\gamma$ leading from a point $\zeta_{0}\left(\right.$ with $\left.0<\left|\zeta_{0}\right|<1\right)$ to the origin $\zeta=0$, such that

$$
\int_{\gamma}|f(\zeta)||d \zeta|<\infty
$$

The first lemma constitutes a strengthened and quantitative form of an argument of R. Osserman [6, p. 397], the second was proved by G. R. MacLane [1]. 
3. The theorem of Osserman and Ahlfors. It was the assumption that the normal domain $P_{0}$ be the unit circle which, in conjunction with the boundedness of the function $\omega(\zeta)$, led to a contradiction before. However, the same contradiction arises already, if the function $\omega(\zeta)$ is expressible as quotient of two bounded analytic functions: $\omega(\zeta)$ $=\psi(\zeta) / \phi(\zeta)(|\phi(\zeta)|<1,|\psi(\zeta)|<1)$ and thus, by a theorem of $\mathrm{R}$. Nevanlinna $[1$, p. 190] is of bounded characteristic. Because then we get

$$
\begin{aligned}
d s & =\left(|\Phi|^{2}+|\Psi|^{2}\right)|d \zeta| \\
& =\left|\frac{\Phi}{\phi}\right|^{2}\left(|\phi|^{2}+|\psi|^{2}\right)|d \zeta| \leqq 2\left|\frac{\Phi}{\phi}\right|^{2}|d \zeta| .
\end{aligned}
$$

If we (and without loss of generality we may do so) assume that the normals omit the direction $(0,0,1)$, the function $\phi(\zeta)$ cannot vanish. From here on the proof proceeds as before.

Now a theorem of O. Frostman [1, p. 101] states that a meromorphic function of bounded characteristic, defined in the unit circle, must be a constant, if its values omit a set of positive logarithmic capacity. Pointing out these facts, L. Ahlfors proved the following theorem which had been conjectured by $\mathrm{R}$. Osserman (see $[\mathbf{5}, \mathrm{p}$. 76]):

A complete minimal surface, whose spherical image omits a set (on the sphere) of positive logarithmic capacity, must be a plane.

Whether there are complete minimal surfaces, different from a plane, whose spherical image actually omits as large a set as one of vanishing capacity, is an open question; see V.1.5 and problem 47.

4. Locally complete minimal surfaces. Using a modification of the first lemma of V.2, R. Osserman showed that

if a minimal surface $S$ is locally complete, i.e., if all paths leading to an isolated boundary component of $S$ have infinite length, then either the normals tend to a single limit along all such paths, or else in every neighborhood of the boundary component the normals assume every direction with the exception of at most a set of logarithmic capacity zero.

This result generalizes qualitatively a well-known theorem of $\mathrm{L}$. Bers [2] to the effect that

the first derivatives of a solution $z(x, y)$ of the minimal surface equation, defined in the exterior of a compact domain of the $(x, y)$-plane, tend to a limit for $x, y \rightarrow \infty:$ For large $x$ and $y$ an expansion

$$
z(x, y)=a x+b y+c \log \left(x^{2}+y^{2}\right)+r(x, y)
$$

holds, where $r(x, y)=O(1)$ and $r_{x}, r_{y}=O\left(\left[x^{2}+y^{2}\right)^{-1 / 2}\right)$. 
Extensions of this theorem to more general equations (2) have been given by L. Bers himself [4], [5] and by R. Finn [4] and H. Jenkins [2]; see also Johannes and Joachim Nitsche [1].

Added in proof. By the method of L. Bers it can also be shown that a solution $z(x, y)$ of the minimal surface equation, defined and bounded in the exterior of a compact domain behaves like $z(x, y)=z_{0}$ $+O\left(\left[x^{2}+y^{2}\right]^{-1 / 2}\right)$ for $x, y \rightarrow \infty$. This remark may be useful for the discussion of the exterior Dirichlet problem for the minimal surface equation.

5. Properties of complete minimal surfaces. The last theorems mark the beginning of far-reaching investigations, in many respects stimulated by $\mathrm{H}$. Hopf, notably by R. Osserman, of the relations which exist between global geometrical and topological and conformal properties of complete minimal surfaces (properties of the spherical image, total curvature, topological type, conformal type, etc.). Exploiting function-theoretical theorems applied to a Weierstrass-type representation of a complete minimal surface, R. Osserman discovered in recent years a number of interesting facts; see [6], [7]. We mention the following ( $S$ always denotes a complete minimal surface):

(1) There are three possibilities: (i) $S$ has infinite total curvature. Then the normals to $S$ assume every direction an infinite number of times with the exception of at most a set of vanishing logarithmic capacity. (ii) $S$ has finite, but not zero total curvature. Then the normals to $S$ assume every direction with at most three exceptions. (iii) $S$ has zero total curvature. Then $S$ is a plane.

(2) If in some neighborhood of the boundary of $S$ the normals omit a set of positive capacity, then $S$ is conformally equivalent to a compact Riemann surface with a finite number of points removed.

(3) If $S$ is either infinitely connected or conformally hyperbolic, then the total curvature of $S$ must be infinite.

(4) If $S$ is finitely connected and conformally parabolic, then either $S$ has finite total curvature or else the normals assume infinitely often every direction with at most two exceptions.

(5) If $S$ has Euler characteristic $\chi$ and $r$ boundary components then the total curvature of $S$ satisfies the inequality

$$
\iint_{S}|K| d o \leqq 2 \pi(x-r) .
$$

This is a strengthened version, valid for minimal surfaces, of the theorem of S. Cohn-Vossen [1] for complete Riemannian 2-manifolds, which states that $\iint_{S}|K| d o \leqq 2 \pi \chi$. 
(6) The total curvature of $S$ is either $-4 \pi n$, for some integer $n \geqq 0$, if $S$ is orientable, or $-2 \pi n$, if $S$ is nonorientable, or else infinite.

(7) The catenoid is the only complete doubly connected minimal surface whose total curvature is equal to $-4 \pi$.

A number of stubborn open questions still remain; see problems 44-47 in Chapter VII.

\section{Chapter VI. Further Results}

1. Connection with boundary-value problems of function theory. I. N. Vekua (in a paper presented at the Joint Soviet-American Symposium on Partial Differential Equations, Novosibirsk, 1963) discussed the realization in space of a simply connected portion of a minimal surface, free of umbilical points, with prescribed value of its Gaussian curvature along the boundary curve. While this problem (which has a multitude of solutions), for a surface assumed in its Weierstrass representation (43), leads to Dirichlet's problem for harmonic functions, I. N. Vekua, working with a somewhat different representation, relates it to an interesting nonlinear boundary-value problem of function theory: To find a function $f(z)$, analytic in $|z| \leqq 1$, for which $\left|f^{\prime}\left(e^{i \theta}\right)\right|=k(\theta)\left(1+\left|f\left(e^{i \theta}\right)\right|^{2}\right)$. Here $k(\theta)$ is a given positive periodic function. Vekua transforms this problem into an integral equation for which he can show the existence of a solution.

Nonlinear boundary-value problems of function theory and their connection with minimal surfaces in Weierstrass's representation have also been discussed by G. Bouligand [1], [2].

2. Plateau's problem for systems of surfaces. Plateau's experiments (see [1] and III.2) did not only concern surfaces bounded by simple closed contours, but, among others, also systems of surfaces spanning more complicated frames. The surface systems in these experiments are bounded partly by free arcs (or branch arcs) along which certain surfaces meet. Thus a cubic frame is found to span 13 surfaces and to have 12 branch arcs, a frame consisting of two coaxial circles is seen to span three surfaces and to have one (closed) branch arc. For a description of such experiments see also D. W. Thompson [1, Chapter VII], R. Courant [1], [4], and R. Courant and H. Robbins [1, pp. 387-389].

While the experiments are quite suggestive, the mathematical difficulties of determining a system of surfaces of least area, bounded by a given frame and certain branch arcs (the shape of which has to be found also) are, even in simple cases, formidable.

Recently, A. D. Solomon [1] attacked the problem in which the frame consists of three rectifiable Jordan $\operatorname{arcs} \Gamma_{1}, \Gamma_{2}, \Gamma_{3}$, joining two 
points $p$ and $q$. The solution here will be a system of three surfaces, each of which is bounded by a branch arc joining $p$ and $q$ and by one of the $\operatorname{arcs} \Gamma_{i}$. Using the methods of R. Courant (see [3, especially Chapter VI]) the existence of a solution is proved. The branch arc is seen to be continuous. In the special case where $\Gamma_{1}$ lies in a plane and $\Gamma_{2}$ and $\Gamma_{3}$ are symmetric with respect to this plane, and assuming that the branch arc lies in this plane and, in fact, that the solution system of surfaces also is symmetric with respect to this plane, A. D. Solomon proves the analyticity of the branch arc in each interior point. The proof uses ideas of R. Courant [2] and H. Lewy [4], [5], [6].

3. Numerical methods. Numerical methods for the solution of Plateau's problem have only rarely been discussed.

For the nonparametric problem, J. Douglas [1], using a nine-point approximation formula, approximates the minimal surface equation by a system of difference equations and designs a step-by-step method for the solution of this system. D. Greenspan [1] replaces the derivatives $z_{x}$ and $z_{y}$ in the area integral $\iint W d x d y$ by difference quotients, so obtaining a functional depending on finitely many parameters. The conditions for an extremal of this functional again lead to a system of difference equations. In both cases the resulting difference equations are nonlinear, a fact which causes numerical difficulties. L. Collatz [1, p. 68] applies his error estimates to check the accuracy of a trial solution in a special case.

The parametric problem is discussed by W. L. Wilson [1], who discretizes the problems of minimizing Dirichlet's integral or Douglas's functional and to a certain degree develops a "discrete theory" of Plateau's problem. His method can also be used for the computation of conformal maps.

The papers of D. Greenspan and W. L. Wilson contain numerical examples and relate experiences concerning the computation. In no case are convergence proofs attempted. ${ }^{8}$ Aside from the necessity of a justification for the numerical methods, convergence proofs would, at the same time, constitute alternate existence proofs for Plateau's problem.

$8 \mathrm{~J}$. Douglas writes in his paper [1, p. 185]: "The spirit of this paper being entirely numerical, we do not concern ourselves with theoretical questions of convergence, which are besides too difficult for us to deal with. Whether convergence occurs or not is indicated practically in each particular case by the behavior of the numbers obtained in the course of the computation." 


\section{Chapter VII. Problems}

The following pages contain a collection of a number of problems and questions, concerning those parts of the theory of minimal surfaces discussed in the previous chapters, which either have defied so far the attempts of their solution or might be worthwhile to be looked into. A few of these problems are of long standing; some have been suggested to me by the work of, or in conversations with, colleagues-R. Courant, R. Finn, H. Jenkins, R. Osserman, T. Radó, J. Serrin, J. E. Thompson, and others; others involve numerical computations. The numbering of the problems corresponds to the order of the preceding paragraphs, and this correspondence is roughly indicated in parentheses.

Suggestions as well as communications concerning treatment of any of these problems would be greatly appreciated.

Added in proof. Aside from the numbered problems listed below, several questions have already been raised in the preceding text. We mention the questions indicated by the remarks at the end of the first paragraph in II.10.1, after formula (23) in II.10.2, and in V.1.6, and the desirability of a thorough study of the "general Scherk surfaces" defined at the end of V.1.5. Also further investigations of Dirichlet's problem with incomplete boundary data (see II.3 and II.12.5) as well as further investigations of various versions of the exterior problem, initiated by L. Bers and Y. W. Chen (see II.1.5 and V.4), are indicated.

1 (II.2.4; II.9.2). In connection with minimal surfaces, which have a simply covered convex curve as central projection upon a plane, the variational problem

$$
\delta \iint e^{2 z}\left[p^{2}+q^{2}+(1+x p+y q)^{2}\right]^{1 / 2} d x d y=0
$$

plays a role. While the variational problem

$$
\delta \iint\left[1+p^{2}+q^{2}\right]^{1 / 2} d x d y=0
$$

has been thoroughly investigated, problem (49) and its Euler equation have not been equally studied. (See T. Radó [5, especially footnote 10 on p. 16].)

2 (II.2). A continued investigation of Dirichlet's problem of the minimal surface equation in nonconvex domains would be desirable. For instance, for nearly plane boundary curves $\Gamma$, A. Korn's conditions 
imply the existence of barriers in boundary points where the boundary is locally mildly nonconvex. Find general facts. How does the Perron solution behave in the neighborhood of a boundary point, in which the boundary is not locally convex? In particular, how does the Perron solution of problem $\mathfrak{P}_{h}$ behave in a neighborhood of the point $C^{\prime}$ ? Is there any relation with a free boundary value problem, where the boundary values are prescribed only over the locally convex portions of the boundary and the solution surface otherwise is required to lie on the cylinder over the boundary? Is there any relation between the Perron solution and the "Bernstein solution" $\phi(x, y)$ of II.2.3 (assuming that it exists), whose values are given on the locally convex portions of the boundary and whose gradient tends to infinity upon approach of the remaining parts of the boundary?

3 (II.5). Find more general sufficient conditions than given in II.5.1, or better still, sufficient and necessary metric conditions on the exceptional set $A$, for the general maximum principle II.5.1 to hold.

4 (II.5). Let $z(x, y)$ be a twice continuously differentiable solution of the minimal surface equation in the unit circle $P=\left\{x, y ; x^{2}+y^{2}<1\right\}$. Simple examples show that the radial limits of $z(x, y)$ for $x^{2}+y^{2} \rightarrow 1$ may be $\pm \infty$ in finitely many points of $\partial P$. Describe the biggest sets on $\partial P$ for which radial limits may not exist. How does $z(x, y)$ behave in general for radial (or angular, etc.) approach to $\partial P$ ? Is there a Fatou type theorem?

5 (II.5; II.3; II.7). With the help of the general maximum principle and a suitable solution of Dirichlet's problem II.7.2 the following maximum principle can be proved:

Let $P$ be a domain (bounded or unbounded) in the $(x, y)$-plane, contained in a sector of opening angle smaller than $\pi$, and let $A$ be a compact set of vanishing linear Hausdorff measure. Let $z(x, y)$ be a solution of the minimal surface equation in $P-A$. Assume that lim $\sup z(x, y)$ $\leqq M(\lim \inf z(x, y) \geqq m)$ for approach of any finite boundary point of $P$, not in $A$. Then the inequality $z(x, y) \leqq M(z(x, y) \geqq m)$ holds in all points of $P-A$.

On the basis of this theorem the existence of a solution of the generalized Dirichlet problem for unbounded domains can be proved.

Can the theorem be generalized to apply to the difference of two solutions, as does the general maximum principle II.5.1 for the case of a bounded domain? If the answer is affirmative, the uniqueness of the solution of the generalized Dirichlet problem for unbounded domains can be proved.

6 (II.6). Find more general sufficient conditions than given in 
II.6, or better still, sufficient and necessary conditions on the exceptional set $A$, for the theorem on isolated singularities II.6 to hold. (See problem 3.)

7 (II.7.4). Let $P$ be a convex quadrilateral. Prove that the condition $l_{1}+l_{3}=l_{2}+l_{4}$ is necessary and sufficient for the existence and uniqueness of a solution of the minimal surface equation in $P$, which tends to $-\infty$ for approach of $\partial P_{1}$ and $\partial P_{3}$ and to $+\infty$ for approach to $\partial P_{2}$ and $\partial P_{4}$ (see Figure 5). Give generalizations to the case of convex $2 n$-polygons with boundary values which are alternatingly $-\infty$ and $+\infty$.

8 (II.9.1). The proofs of Bernstein's theorem by S. Bernstein [4], E. Hopf [1], E. J. Mickle [2], and an intricate proof by W. H. Fleming $[3$, p. 83] do not use function theory. Find such a proof which is as simple as possible.

9 (II.9.5). Determine (or, at least, give a better estimate for) the constant $c_{1}^{(0)}$.

10 (II.9.5). Determine (or, at least, give a better estimate for) the constant $c_{2}^{(0)}$.

11 (II.10.1). Determine (or, at least, give a better estimate for) the constant $c_{3}^{(0)}$.

12 (II.10.1). Determine (or, at least, give a better estimate for) the constant $c_{4}^{(0)}$.

13 (II.10.1). Determine (or, at least, give a better estimate for) the constants $c_{5}^{(0)}$ and $c_{6}^{(0)}$.

14 (II.10.1). Determine the function $R(\rho)$ for $0<\rho<1$.

15 (II.12.1). Determine (or, at least, give a better estimate for) the constant $c_{7}^{(0)}$.

16 (II.12.2). Determine the constant $c_{8}^{(0)}$.

17 (II.12.2). Estimate (27) could at least be improved by the use of more sophisticated comparison surfaces than Scherk's, for instance, the solution of the minimal surface equation (i.e., the solution of Dirichlet's problem discussed in II.7.2) in a polygonal region, bounded by a chord of the unit circle and a polygonal arc on this chord inscribed into the unit circle, with values zero on the polygonal arc and $+\infty$ on the chord. While the determination of this surface is amenable to the classical methods, its explicit representation and applicability for numerical estimates seem not easy to secure.

18 (II.12.2). Still more useful, but probably still harder to come by, would be the solution of the minimal surface equation in a circular segment with values zero on the circular arc of the boundary and $+\infty$ on the chord, or at least sharp estimates for this solution and its first derivatives. 
19 (II.12.2). Similarly, in connection with estimate (28), much information would be desirable about the solution of the minimal surface equation in a disc with values $+M$ on a subarc of the boundary (in particular, a semi-circle) and $-M$ on the complementary arc.

20. Improve estimate (31).

21 (II.12.5). Let $z_{1}(x, y), z_{2}(x, y) \in C^{2}(P) \cap C^{0}(\bar{P})$ be two solutions of the minimal surface equation in a bounded convex (not necessarily strictly convex) domain. Let $\left(x_{0}, y_{0}\right)$ be an interior point of this domain. Does an estimate of the form

$$
\left|z_{2}\left(x_{0}, y_{0}\right)-z_{1}\left(x_{0}, y_{0}\right)\right| \leqq c \int_{\partial P}\left|z_{2}-z_{1}\right| d s
$$

(or a similar estimate) hold, where the constant $c$ depends on the point $\left(x_{0}, y_{0}\right)$, the domain $P$, and the $L^{1}$-norm of the boundary values of $z_{1}$ and $z_{2}$ only?

22 (III.2). Find new conditions on the Jordan curve $\Gamma$ which guarantee the uniqueness of a minimal surface (or a generalized minimal surface) of the type of the disc, bounded by $\Gamma$.

23 (III.2). Give an example of a Jordan curve spanning (at least) two different minimal surfaces of the type of the disc, both explicitly known.

24 (III.3.2). Give as simple a proof as possible of the isoperimetric inequality for saddle surfaces of finite area of the type of the disc, bounded by a rectifiable Jordan curve.

25 (III.3.3). Let $S$ be a minimal surface (or a generalized minimal surface) of the type of the circular annulus, bounded by two rectifiable Jordan curves $\Gamma_{1}$ and $\Gamma_{2}$. Give an estimate for the area $I(S)$ of $S$ in terms of the lengths of $\Gamma_{1}$ and $\Gamma_{2}$. If necessary, consider special cases (additional regularity assumptions, boundary curves in parallel planes, surface given in conformal representation, etc.). By IV.2 the existence of $S$ implies that the curves $\Gamma_{1}$ and $\Gamma_{2}$ cannot be too far apart. Is there an isoperimetric type inequality? Is there a relation between $I(S)$, the lengths of the curves $\Gamma_{i}$, and the areas of minimal surfaces $S_{1}, S_{2}$ of the type of the disc bounded by $\Gamma_{1}$ and $\Gamma_{2}$, respectively?

26 (III.3.3). Let $S$ be a minimal surface (or a generalized minimal surface) of given topological type, bounded by $r$ rectifiable Jordan curves $\Gamma_{i}$. Give an estimate for the area of $S$ in terms of the lengths of the curves $\Gamma_{i}$. If necessary, consider special cases (additional regularity assumptions, surface given in conformal representation, etc.).

27 (III.4). Find new conditions for the Jordan curve $\Gamma$, such that any generalized minimal surface (possibly in a conformal representa- 
tion) of the type of the disc, bounded by $\Gamma$, is a minimal surface, i.e., will not have (interior) branch points.

28 (III.4). Give conditions on the curves $\Gamma_{1}, \Gamma_{2}, \cdots, \Gamma_{r}$, such that any generalized minimal surface (possibly in a conformal representation) of prescribed topological type, bounded by the curves $\Gamma_{i}$, is a minimal surface, i.e., will not have (interior) branch points.

29 (III.4.4). Is there a Jordan curve $\Gamma$ in space with the property, that any generalized minimal surface (possibly in a conformal representation) of the type of the disc, bounded by $\Gamma$, must have an (interior) branch point?

30 (III.4.4). Is it true that a reasonable (analytic, differentiable, rectifiable, polygonal,... ?) Jordan curve $\Gamma$, knotted or not, always bounds a minimal surface of the type of the disc, i.e., a surface without (interior) branch points?

31 (III.5.2). Let $\Gamma$ be a Jordan curve, bounding a generalized or proper minimal surface $S=\{\mathfrak{x}=\mathfrak{x}(u, v) ;(u, v) \in \bar{P}\}, P$ the unit circle, of the type of the disc (possibly in a conformal representation, possibly a surface of least area). Let $\Gamma_{0}$ be an open subarc of $\Gamma$, possibly all of $\Gamma$, corresponding to the arc $\gamma_{0}$ of $P$. Under various regularity assumptions (short of analyticity) about the arc $\Gamma_{0}$ find estimates for the derivatives of the vector $\mathfrak{x}(u, v)$ in $P \cup \gamma_{0}$. For an analytic arc $\Gamma_{0}$ find estimates for the derivatives of the vector $\mathfrak{x}(u, v)$ in $P \cup \gamma_{0}$ in terms of geometrical invariants of the arc $\Gamma_{0}$ and of finitely many derivatives of its vector.

32 (IV.1). For minimal surfaces of the type of the circular annulus bounded by two regular $n$-polygons, inscribed into coaxial unit circles in parallel planes of distance $r=2 h$, compute the value $h_{2}(n)$, which is defined in the same way as $h_{2}$ in IV.1. The value $h_{1}(n)$, particularly for the case $n=4$, has been discussed by H. A. Schwarz [4]. We should have $\lim _{n \rightarrow \infty} h_{2}(n)=h_{2}$.

33 (IV.1). Using the explicit representation in terms of elliptic integrals of minimal surfaces of the type of the annulus, bounded by two unit circles in parallel planes of distance $r=2 h$ (see B. Riemann [1, pp. 329-333], A. Enneper [2], J. C. C. Nitsche [10]) compute the function $d_{2}(h)$, defined in IV.1 and the end of IV.2. Among other things this involves a determination of parameters, and the task is of great computational difficulty. $d_{2}(h)$ is defined for $0<h \leqq h_{2}$. Probably $d_{2}(h)$ is monotonically decreasing, and $d_{2}\left(h_{2}\right)=0, \lim _{h \rightarrow 0} d_{2}(h) \geqq 1$.

34 (IV.2). Sharpen and, if possible, generalize estimates (38) and (39).

35 (IV.3). Are there theorems in the spirit of Shiffman's results IV.3 for other types of curves $\Gamma_{1}$ and $\Gamma_{2}$, for instance star-shaped curves (possibly satisfying additional conditions)? 
36 (IV.4). For minimal surfaces of class $S(r)$ give an estimate of the type of (40) in terms of the distance $d$ of the curve $S_{0}$ from the boundary of $S$ instead $r$.

37 (IV.4). Is an estimate of the type of (40) valid for minimal surfaces of class $\Im(r)$ ?

38 (IV.4). Is every minimal surface of class $\Im(\infty)$ a catenoid?

39 (IV.4). Is every complete minimal surface of class $\Im(r)$ a catenoid?

40 (IV.4; IV.5). Find theorems in the spirit of those proved in IV.4 and IV. 5 characterizing special (at least, complete) minimal surfaces other than catenoid or helicoid.

41 (IV.5). Give proofs of K. Leichtweiss's theorems under different or reduced assumptions; in particular, try to weaken or omit assumptions 2 or 3 . Give quantitative versions in the spirit of the theorem in IV.4.

42 (V.4). Give as simple a proof as possible (preferably one using little function theory) of the theorem of L. Bers to the effect that the first derivatives of a solution $z(x, y)$ of the minimal surface equation, defined in the exterior of a compact domain of the $(x, y)$-plane, tend to a limit for $x, y \rightarrow \infty$.

43 (V.5). For the proof of Theorem 2 in V.5 (as well as for other theorems) R. Osserman uses the following theorem of A. Huber (see [3, Theorem 13, p. 61]):

Suppose that the conformal metric $e^{u(z)}|d z|$, defined on an infinitely connected Riemann surface $S$, is complete. Then $\iint_{S} K^{-} d o=\infty$.

(Here $K^{-}=\operatorname{Max}(-K, 0)$.) Huber's proof of this theorem is quite intricate. Considering that in its application to minimal surfaces only the special case is needed, where the Gaussian curvature of the surface has compact support, it would be desirable to have a short proof of Huber's Theorem 13 for this case.

44 (V.1.5; V.5). Is the covering surface $R_{0}-$ parameter domain of Scherk's surface $\left(6^{\prime}\right)$ described at the end of V.1.5 - of conformal hyperbolic or parabolic type?

45 (V.5). Do there exist complete nonorientable minimal surfaces?

46 (V.5). Is there a complete minimal surface of finite total curvature whose normals omit exactly three directions?

47 (V.5). There are examples of complete minimal surfaces, different from a plane (of infinite total curvature), whose normals omit exactly four directions; see V.1.5. Are there such surfaces, whose normals omit more than four or exactly five directions, or, more generally, exactly a set of prescribed directions of vanishing capacity on the sphere? 
48 (VI.2). Discuss the problem of A. D. Solomon under fewer assumptions about the symmetry of the solution system of surfaces and its branch line.

49 (VI.2). Discuss other cases of Plateau's problem for systems of surfaces.

50 (VI.3). Discuss methods for the numerical solution of the (parametric and nonparametric) Plateau problem and-most importantgive convergence proofs.

\section{BIBLIOGRAPHY}

E. F. BECKENBACH 664.

1. The area and boundary of minimal surfaces, Ann. of Math. (2) 33 (1932), 658-

E. F. Beckenbach and G. A. Hutchison

1. Meromorphic minimal surfaces, Bull. Amer. Math. Soc. 68 (1962), 519-522.

E. F. Beckenbach and T. Radó

1. Subharmonic functions and minimal surfaces, Trans. Amer. Math. Soc. 35 (1933), 648-661.

2. Subharmonic functions and surfaces of negative curvature, Trans. Amer. Math. Soc. 35 (1933), 662-664.

E. BeLTRAMI

1. Sulle proprietd generali delle superficie ad area minima, Mem. Accad. Bologna (2) 7 (1867), 411-481.

P. BERG

1. On univalent mappings by solutions of linear elliptic partial differential equations, Trans. Amer. Math. Soc. 84 (1957), 310-318.

S. BERNSTEIN

1. Sur la généralisation du problème de Dirichlet. II, Math. Ann. 69 (1910), 82-136.

2. Sur les équations du calcul des variations, Ann. Sci. Ecole Norm. Sup. (3) 29 (1912), 431-485.

3. Sur l'intégration des équations aux dérivées partielles du type elliptique, Math. Ann. 95 (1926), 585-594.

4. Über ein geometrisches Theorem und seine Anwendung auf die partiellen Differentialgleichungen vom elliptischen Typus, Math. Z. 26 (1927), 551-558.

L. BERS

1. Boundary value problems for minimal surfaces with singularities at infinity, Trans. Amer. Math. Soc. 70 (1951), 465-491.

2. Isolated singularities of minimal surfaces, Ann. of Math. (2) 53 (1951), 364-386.

3. Abelian minimal surfaces, J. Analyse Math. 1 (1951), 43-48.

4. Non-linear elliptic equations without non-linear entire solutions, J. Rational Mech. Anal. 3 (1954), 767-787.

5. Function-theoretical properties of solutions of partial differential equations of elliptic type, pp. 69-94, Annals of Mathematics Studies No. 33, Princeton Univ. Press, Princeton, N. J., 1954.

L. BIANCHI

1. Vorlesungen über Differentialgeometrie, Teubner Verlag, Leipzig, 1899.

W. BLASCHKE AND H. REICHARDT

1. Einführung in die Differentialgeometrie, 2nd ed., Springer-Verlag, Heidelberg, 1960. 
G. A. BuIss

1. Calculus of variations, Open Court, La Salle, 1925.

G. Bouligand

1. Sur quelques problèmes fonctionnels non linéaires, C. R. Acad. Sci. Paris 241 (1955), 1537-1539.

2. Surfaces minima et operateurs linéaires associés, C. R. Acad. Sci. Paris 241 (1955), 1676-1678.

C. V. Boys

1. Soap bubbles: their colours and the forces which mold them, Dover, New York, 1959.

E. CALABI

1. Improper affine hyperspheres of convex type and a generalization of a theorem by K. Jörgens, Michigan Math. J. 5 (1958), 105-126.

J. W. Calkin

1. Functions of several variables and absolute continuity. I, Duke Math. J. 6 (1940), 170-186.

T. Carleman

1. Zur Theorie der Minimalflächen, Math. Z. 9 (1921), 154-160.

L. Carleson

1. Selected problems on exceptional sets, (Mimeographed), Uppsala, 1961.

E. Catalan

1. Mémoire sur les surfaces dont les rayons de courbure, en chaque point, sont égaux et de signes contraires, J. Ecole Polytech. 21 (1858), 129-168.

L. Cesari

1. Surface area, Princeton Univ. Press, Princeton, N. J., 1956.

S. A. Chaplygin

1. On gas jets, Sci. Mem. Imp. Univ. Moscow Math. Phys. Sect. 21 (1902), 1-121. (Russian)

Y. W. CHEN

1. Branch points, poles, and planar points of minimal surfaces in $R^{3}$, Ann. of Math. (2) 49 (1948), 790-806.

2. Existence of minimal surfaces with a pole at infinity and condition of transversality on the surface of a cylinder, Trans. Amer. Math. Soc. 65 (1949), 331-347.

3. Discontinuity and representations of minimal surface solutions, pp. 115-138, Proceedings Conference on Differential Equations, Univ. of Maryland, College Park, Md., 1956.

S. CoHN-Vossen

1. Kürzeste Wege und Totalkrümmung auf Flächen, Compositio Math. 2 (1935), 69-133.

L. Collatz

1. Sätze über monotones Verhalten bei gewöhnlichen und partiellen Differentialgleichungen, pp. 61-80, Colloque sur l'Analyse Numérique, tenu à Mons les 22, 23 et 24 Mars 1961, Gauthier-Villars, Paris, 1961.

R. Courant

1. Soap film experiments with minimal surfaces, Amer. Math. Monthly 47 (1940), 167-174.

2. On Plateau's problem with free boundaries, Proc. Nat. Acad. Sci. U.S.A. 31 (1945), 242-246.

3. Dirichlet's principle, conformal mapping, and minimal surfaces, Interscience, New York, 1950. 
4. Unsolved problems concerning least area (with experimental demonstrations), pp. 305-307, Outlines Joint Soviet-American Sympos. on Partial Differential Equations, Novosibirsk, 1963.

R. Courant and D. Hilbert

1. Methods of mathematical physics, Vol. II, Interscience, New York, 1962.

R. Courant and H. Robbins

1. What is mathematics?, 2nd ed., Oxford Univ. Press, London, 1943.

H. L. DE VRIES

1. A remark concerning a lemma of Heinz on harmonic mappings, J. Math. Mech. 11 (1962), 469-471.

J. Douglas

1. A method of numerical solution of the problem of Plateau, Ann. of Math. (2) 29 (1928), 180-188.

2. Solution of the problem of Plateau, Trans. Amer. Math. Soc. 33 (1931), 263-321.

3. The problem of Plateau for two contours, J. Math. and Phys. 10 (1931), 315-359.

4. The problem of Plateau, Bull. Amer. Math. Soc. 39 (1933), 227-251.

5. Minimal surfaces of general topological structure with any finite number of assigned boundaries, J. Math. and Phys. 15 (1936), 105-123.

6. Minimal surfaces of higher topological structure, Ann. of Math. (2) 40 (1939), 205-298.

7. The most general form of the problem of Plateau, Amer. J. Math. 61 (1939), 590-608.

A. ENNEPER

1. Analytisch-geometrische Untersuchungen, Z. Math. Phys. 9 (1864), 96-125.

2. Die cyklischen Flächen, Z. Math. Phys. 14 (1869), 393-421.

W. FENCHEL

1. Über Krümmung und Windung geschlossener Raumkurven, Math. Ann. 101 (1929), 238-252.

R. FINN

1. A property of minimal surfaces, Proc. Nat. Acad. Sci. U.S.A. 39 (1953), 197201.

2. Isolated singularities of solutions of non-linear partial differential equations, Trans. Amer. Math. Soc. 75 (1953), 385-404.

3. On equations of minimal surface type, Ann. of Math. (2) 60 (1954), 397-416.

4. On a problem of type, with application to elliptic partial differential equations, J. Rational Mech. Anal. 3 (1954), 789-799.

5. Growth properties of solutions of non-linear elliptic equations, Comm. Pure Appl. Math. 9 (1956), 415-423.

6. New estimates for equations of minimal surface type, Arch. Rational Mech. Anal. 14 (1963), 337-375.

7. Remarks relevant to minimal surfaces, Tech. Rep. No. 120, Appl. Math. and Statist. Lab., Stanford University, Stanford, Calif., 1963.

8. Remarks on my paper "On equations of minimal surface type," Ann. of Math. (2) 80 (1964), 158-159.

R. FINN AND R. OsSERMAN

1. On the Gauss curvature of non-parametric minimal surfaces, J. Analyse Math. 12 (1964), 351-364.

H. FLANDERS

1. On certain functions with positive definite Hessian, Ann. of Math. (2) 71 (1960), 153-156. 


\section{W. H. FLEMiNG} 1074.

1. An example in the problem of least area, Proc. Amer. Math. Soc. 7 (1956), 1063-

2. Nondegenerate surfaces of finite topological type, Trans. Amer. Math. Soc. 90 (1959), 323-335.

3. On the oriented Plateau problem, Rend. Circ. Mat. Palermo (2) 11 (1962), 69-90. M. FRÉCHET

1. Détermination des surfaces minima du type $a(x)+b(y)=c(z)$, Rend. Circ. Mat. Palermo (2) 5 (1956), 238-259; (2) 6 (1957), 5-32.

O. Frostman

1. Potentiel d'équilibre et capacité des ensembles avec quelques applications a la théorie des fonctions, Medd. Lunds Univ. Mat. Sem. 3 (1935), 1-118.

P. Funk

1. Über zweidimensionale Finslersche Räume, insbesondere über solche mit geradlinigen Extremalen und positiver konstanter Krïmmung, Math. Z. 40 (1935), 86-93.

2. Eine Kennzeichnung der zweidimensionalen elliptischen Geometrie, Österreich. Akad. Wiss. Math.-Natur. Kl. S.-B. (to appear).

R. GARNIER

1. Sur le problème de Plateau pour un quadrilatère variable qui peut acquêrir un point double, C. R. Acad. Sci. Paris 251 (1960), 171-174; Ann. Mat. Pura Appl. (4) 58 (1962), 1-34.

2. Sur le problème de Plateau pour les quadrilatères gauches avant un sommet $a$ l'infini, C. R. Acad. Sci. Paris 254 (1962), 2260-2264.

H. GRAF UND H. Thomas

1. Zur Frage des Gleichgewichts von Vierecksnetzen aus verknoteten und gespannten Fäden. II, Math. Z. 51 (1948), 166-196.

D. GREENSPAN

1. On approximating extremals of functionals, I. The method and examples for boundary value problems, Tech. Rep. No. 466, Math. Research Center, Univ. of Wisconsin, Madison, Wis., 1964.

A. HAAR 1-18.

1. Über die Variation der Doppelintegrale, J. Reine Angew. Math. 149 (1919),

2. Über das Plateausche Problem, Math. Ann. 97 (1927), 124-258.

3. Über adjungierte Variationsprobleme und adjungierte Extremalfä̈chen, Math. Ann. 100 (1928), 481-502.

E. HEINZ

1. Über die Lösungen der Minimalfä̈chengleichung, Nachr. Akad. Wiss. Göttingen Math.-Phys. Kl. II (1952), 51-56.

2. Über gewisse elliptische Systeme von Differentialgleichungen zweiter Ordnung mit Anwendung auf die Monge-Ampère'sche Gleichung, Math. Ann. 131 (1956), 411-428.

3. On certain nonlinear elliptic differential equations and univalent mappings, J. Analyse Math. 5 (1956/1957), 197-272.

4. On one-to-one harmonic mappings, Pacific J. Math. 9 (1959), 101-105.

L. HENNEBERG

1. Über solche Minimalflächen, welche eine vorgeschriebene ebene Curve zur geodatischen Linie haben, Doctoral Dissertation, Eidgenössisches Polytechnikum, Zürich, 1875.

2. Über diejenige Minimalflächen, welche die Neil'sche Parabel zur ebenen geodätischen Linie hat, Vierteljschr. Naturforsch. Ges. Zürich 21 (1876), 66-70. 
A. HerzoG

1. Bestimmung einiger speziellen Minimalflächen, Vierteljchr. Naturforsch. Ges. Zürich 20 (1875), 217-254.

D. Hilbert AND S. Cohn-Vossen

1. Geometry and the imagination, Chelsea, New York, 1952.

E. HopF

1. On S. Bernstein's theorem on surfaces $z(x, y)$ of non-positive curvature, Proc. Amer. Math. Soc. 1 (1950), 80-85.

2. On an inequality for minimal surfaces $z=z(x, y)$, J. Rational Mech. Anal. 2 (1953), 519-522; 801-802.

C. C. Hsiung

1. Isoperimetric inequalities for two-dimensional Riemannian manifolds with boundary, Ann. of Math. (2) 73 (1961), 213-220.

A. HUBER

1. On the isoperimetric inequality on surfaces of variable Gaussian curvature, Ann. of Math. (2) 60 (1954), 237-247.

2. Zur isoperimetrischen Ungleichung auf gekrümmten Flächen, Acta Math. 97 (1957), 95-101.

3. On subharmonic functions and differential geometry in the large, Comment. Math. Helv. 32 (1957), 13-72.

H. JENKINS

1. On two-dimensional variational problems in parametric form, Arch. Rational Mech. Anal. 8 (1961), 181-206.

2. On quasi-linear elliptic equations which arise from variational problems, J. Math. Mech. 10 (1961), 705-728.

3. Super solutions for quasi-linear elliptic equations, Arch. Rational Mech. Anal. 16 (1964), 402-410.

H. JENKINS AND J. SERRIN

1. Variational problems of minimal surface type. I, Arch. Rational Mech. Anal 12 (1963), 185-212.

H. JONAS

1. Die Scherksche Minimalfäche als Gegenstand einer anschaulichen geometrischen Deutung des Additionstheorems für das elliptische Integral 1. Gattung, Math. Nachr. 8 (1952), 41-52.

K. JöRGENS

1. Über die Lösungen der Differentialgleichung $r t-s^{2}=1$, Math. Ann. 127 (1954), 130-134.

2. Harmonische Abbildungen und die Differentialgleichung $r t-s^{2}=1$, Math. Ann. 129 (1955), 330-344.

J. B. KELLER

1. The steepest minimal surface over the unit circle, Arch. Rational Mech. Anal. 14 (1963), 372-374.

A. KORN

1. Über Minimalflächen, deren Randkurven wenig von ebenen Kurven abweichen, Abh. König. Preuss. Akad. Wiss. Berlin Phys.-Math. Cl. II (1909), 1-37.

M. KRUSKAL

1. The bridge theorem for minimal surfaces, Comm. Pure Appl. Math. 7 (1954), 297-316.

J. LELONG-FERRAND

1. Représentation conforme et transformations a intégrale de Dirichlet bornée, Gauthier-Villars, Paris, 1955. 


\section{J. LERAY}

1. Discussion d'un probleme de Dirichlet, J. Math. Pures Appl. 18 (1939), 249-284. P. LEVY

1. Exemples de contours pour lesquels le problème de Plateau a 3 ou $2 p+1$ solutions, C. R. Acad. Sci. Paris 224 (1947), 325-327.

2. Le problème de Plateau, Mathematica 23 (1948), 1-45.

H. LEWY

1. On the nonvanishing of the Jacobian in certain one-to-one mappings, Bull. Amer. Math. Soc. 42 (1936), 689-692.

2. A priori limitations for solutions of Monge-Ampère equations. II, Trans. Amer. Soc. 41 (1937), 365-374.

3. A property of spherical harmonics, Amer. J. Math. 60 (1938), 555-560.

4. A note on harmonic functions and a hydrodynamical application, Proc. Amer. Math. Soc. 3 (1951), 111-113.

5. On the boundary behavior of minimal surfaces, Proc. Nat. Acad. Sci. U.S.A. 37 (1951), 103-110.

6. On minimal surfaces with partially free boundary, Comm. Pure Appl. Math. 4 (1951), 1-13.

S. LIE

1. Beiträge zur Theorie der Minimalfächen, Math. Ann. 14 (1879), 331-411.

R. v. LILIENTHAL

1. Besondere Flächen, Encyklopädie der mathematischen Wissenschaften, Vol. 3, Part 3.D, pp. 269-354, Teubner, Leipzig, 1902-1927.

S. LozINSKY

1. On subharmonic functions and their applications to the theory of surfaces, Bull. Acad. Sci. SSSR. Sér. Math. 8 (1944), 175-194. (Russian. English Summary)

G. R. MAcLane

1. On asymptotic values, Abstract 603-166, Notices Amer. Math. Soc. 10 (1963), 482-483.

I. MARX

1. On the classification of unstable minimal surfaces with polygonal boundaries, Comm. Pure Appl. Math. 8 (1955), 235-244.

E. J. McShane

1. Parametrization of saddle surfaces with applications to the problem of Plateau, Trans. Amer. Math. Soc. 35 (1933), 716-733.

2. On the analytic nature of surfaces of least area, Ann. of Math. (2) 35 (1934), 456473.

E. J. MickLE

1. Associated double integral variation problems, Duke Math. J. 9 (1942), 208-227. 86-89.

2. A remark on a theorem of Serge Bernstein, Proc. Amer. Math. Soc. 1 (1950), J. MILNOR

1. On the total curvature of knots, Ann. of Math. (2) 52 (1950), 248-257.

C. B. MORREY

1. An analytic characterization of surfaces of finite Lebesgue area, Amer. J. Math. 57 (1935), 692-702; 58 (1936), 313-332.

2. Functions of several variables and absolute continuity. II, Duke Math. J. 6 (1940), 187-215.

3. The problem of Plateau on a Riemannian manifold, Ann. of Math. (2) 49 (1948), 807-851. 
C. H. MÜNTZ

1. Die Lösung des Plateauschen Problems ïber konvexen Bereichen, Math. Ann. 94 (1925), 53-96.

2. Zum Plateauschen Problem. Erwiderung auf die vorstehende Note des Herrn Rado, Math. Ann. 96 (1927), 597-600.

E. R. NEOVIUS

1. Bestimmung zweier speziellen periodischen Minimalfächen, auf welchen unendlich viele gerade Linien und unendlich viele ebene geodätische Linien liegen, Akad. Abh., Frenkell, Helsingfors, 1883.

R. NEVANLINNA

1. Eindeutige analytische Funktionen, 2nd ed., Springer-Verlag, Heidelberg, 1953. J. C. C. NitsCHE

1. Über eine mit der Minimalfä̈chengleichung zusammenhängende analytische Funktion und den Bernsteinschen Satz, Arch. Math. 7 (1956), 417-419.

2. Elementary proof of Bernstein's theorem on minimal surfaces, Ann. of Math. (2) 66 (1957), 543-544.

3. A uniqueness theorem of Bernstein's type for minimal surfaces in cylindrical coordinates, J. Math. Mech. 6 (1957), 859-864.

4. Minimal surfaces, Mimeographed Lecture Outlines, Univ. of Minnesota, Minneapolis, Minn., 1957/1958.

5. On harmonic mappings, Proc. Amer. Math. Soc. 9 (1958), 268-271.

6. Zu einem Satze von L. Bers über die Lösungen der Minimalfä̈chengleichung, Arch. Math. 9 (1958), 427-429.

7. On an estimate for the curvature of minimal surfaces $z=z(x, y)$, J. Math. Mech. 7 (1958), 767-769.

8. On the constant of E. Heinz, Rend. Circ. Mat. Palermo (2) 8 (1959), 178-181.

9. Ein Eindeutigkeitssatz für zweifach zusammenhängende Minimalfächen, Math. Z. 74 (1960), 289-292.

10. A characterization of the catenoid, J. Math. Mech. 11 (1962), 293-302.

11. On the module of doubly-connected regions under harmonic mappings, Amer. Math. Monthly 69 (1962), 781-782.

12. Über die Ausdehnung gewisser zweifach zusammenhängender Minimalfächen, Math. Ann. 149 (1963), 144-149.

13. On the total curvature of a closed curve, MR 25 \#492 (1963), 104.

14. Zum Heinzschen Lemma ïber harmonische Abbildungen, Arch. Math. 14 (1963), 407-410.

15. A necessary criterion for the existence of certain minimal surfaces, J. Math. Mech. 13 (1964), 659-666.

16. Über ein verallgemeinertes Dirichletsches Problem für die Minimalfächengleichung und hebbare Unstetigkeiten ihrer Lösungen, Math. Ann. (to appear).

17. A supplement to the condition of $J$. Douglas, Rend. Circ. Mat. Palermo (to appear).

18. On the non-solvability of Dirichlet's problem for the minimal surface equation, J. Math. Mech. (to appear).

19. On differential equations of mode 2, Proc. Amer. Math. Soc. (to appear). JoHANNES Nitsche AND JoACHIM Nitsche

1. Ein Kriterium für die Existenz nicht-linearer ganzer Lösungen elliptischer Differentiagleichungen, Arch. Math. 10 (1959), 294-297. 353.

2. Über reguläre Variationsprobleme, Rend. Circ. Mat. Palermo (2) 8 (1959), 346- 


\section{R. Osserman}

1. Proof of a conjecture of Nirenberg, Comm. Pure Appl. Math. 12 (1959), 229-232.

2. Remarks on minimal surfaces, Comm. Pure Appl. Math. 12 (1959), 233-239.

3. An analogue of the Heinz-Hopf inequality, J. Math. Mech. 8 (1959), 383-385.

4. On the Gauss curvature of minimal surfaces, Trans. Amer. Math. Soc. 96 (1960), 115-128.

5. Minimal surfaces in the large, Comment. Math. Helv. 35 (1961), 65-76.

6. On complete minimal surfaces, Arch. Rational Mech. Anal. 13 (1963), 392-404.

7. Global properties of minimal surfaces in $E^{3}$ and $E^{n}$, Ann. of Math. (2) 80 (1964), 340-364.

8. Global properties of classical minimal surfaces, Tech. Rep. No. 30, Department of Math., Stanford Univ., Stanford, Calif., 1964.

O. Perron

1. Eine neue Behandlung der Randwertaufgabe fïr $\Delta u=0$, Math. Z. 18 (1923), 42-54.

J. Plateau

1. Statique experimentale et théorique des liquides soumis aux seules forces moléculaires, Gauthier-Villars, Paris, 1873.

S. D. Porsson

1. Note sur le surface dont l'aire est un minimum entre des limites données, J. Reine Angew. Math. 8 (1832), 361-362.

T. RADó

1. Bemerkung ïber die Differentialgleichungen zweidimensionaler Variationsprobleme, Acta Litt. Sci. Univ. Szeged 3 (1925), 147-156.

2. Zu einem Satze von S. Bernstein über Minimalfächen in Grossen, Math. Z. 26 (1927), 559-565.

3. Bemerkung zur Arbeit von Herrn. Ch. H. Müntz über das Plateausche Problem, Math. Ann. 96 (1927), 587-596.

4. The problem of the least area and the problem of Plateau, Math. Z. 32 (1930), 763-796.

5. Contributions to the theory of minimal surfaces, Acta Litt. Sci. Univ. Szeged 6 (1932), 1-20.

6. On the problem of Plateau, Ergebnisse der Mathematik und ihrer Grenzgebiete, Springer-Verlag, Berlin, 1933.

7. Length and area, Amer. Math. Soc. Colloq. Publ. Vol. 30, Amer. Math. Soc., Providence, R. I., 1948.

M. O. READE

1. A characterization of minimal surfaces in isothermic representation, Proc. Amer. Math. Soc. 2 (1951), 47-54.

R. M. REDHEFFER

1. On pairs of harmonic functions, Proc. Amer. Math. Soc. 8 (1957), 450-457.

W. T. REID

1. The isoperimetric inequality and associated boundary problems, J. Math. Mech. 8 (1959), 897-905.

H. W. RICHMOND

1. On minimal surfaces, J. London Math. Soc. 19 (1944), 229-241.

B. RiEmanN

1. Über die Fläche von kleinstem Inhalt bei gegebener Begrenzung, 2nd ed., Ges. Math. Werke, Teubner, Leipzig, 1892; pp. 301-333. 
F. RIESZ UND M. RIESZ

1. Über die Randwerte einer analytischen Funktion, Quatrième Congrés des Math. Scand., Stockholm, 1916; pp. 27-44.

I. F. RitTER

1. Solution of Schwarz' problem concerning minimal surfaces, Univ. Nac. Tucumán Rev. Ser. A 1 (1940), 40-62.

S. SASAKI

1. On the total curvature of a closed curve, Japan. J. Math. 29 (1959), 118-125.

H. F. SCHERK

1. Bemerkungen über die kleinste Fläche innerhalb gegebener Grenzen, J. Reine Angew. Math. 13 (1835), 185-208.

H. A. SCHWARZ

1. Bestimmung einer speziellen Minimalfläche, Vol. 1, Ges. Math. Abh., SpringerVerlag, Berlin, 1890; pp. 6-125.

2. Miscellen aus dem Gebiet der Minimalfächen, Vol. 1, Ges. Math. Abh., SpringerVerlag, Berlin, 1890; pp. 169-189.

3. Über diejenigen Minimalflächen, welche von einer Schaar von Kegeln zweiten Grades eigehiullt werden, Vol. 1, Ges. Math. Abh., Springer-Verlag, Berlin, 1890; pp. 190-204.

4. Ueber spezielle zweifach zusammenhängende Flächenstïcke, welche kleineren Flächeninhalt besitzen, als alle benachbarten, von denselben Randlinien begrenzten Flächenstiicke, Vol. 1, Ges. Math. Abh., Springer-Verlag, Berlin, 1890; pp. 270-316. C. Z. ŠEFL'

1. Investigations on the geometry of saddle surfaces, Akad. Nauk SSSR Sibirsk, 1963. (Russian)

B. SEGRE

1. Questioni di realtd sulle forme armoniche e sulle loro hessiane, Atti Accad. Naz. Lincei Rend. Cl. Sci. Fis. Mat. Natur. (8) 15 (1953), 237-242; (8) 15 (1954), 339-344.

2. Questioni di realtd sulle forme armoniche ternarie e sulle loro hessiane, pp. 148151, Convegno Internazionale di Geometria Differenziale, Italia, 1953, Edizioni Cremonese, Roma, 1954.

J. SERRIN

1. Dirichlet's principle in the calculus of variations, Proc. Sympos. Pure Math., Vol. 4, pp. 17-22, Amer. Math. Soc., Providence, R. I., 1961.

2. A priori estimates for solutions of the minimal surface equation, Arch. Rational Mech. Anal. 14 (1963), 376-383.

3. Local behavior of solutions of quasi-linear equations, Acta Math. 111 (1964), 247-302.

K. SHibata

1. On the existence of a harmonic mapping, Osaka Math. J. 15 (1963), 173-211.

M. SHIFFMAN

1. The Plateau problem for minimal surfaces which are relative minima, Ann. of Math. (2) 39 (1938), 309-315.

2. The Plateau problem for minimal surfaces of arbitrary topological structure, Amer. J. Math. 61 (1939), 853-882.

3. On surfaces of stationary area bounded by two circles, or convex curves, in parallel planes, Ann. of Math. (2) 63 (1956), 77-90.

A. G. Sigalov

1. Two-dimensional problems of the calculus of variations, Amer. Math. Soc. Transl. (1) 6 (1953), 27-146. 
A. D. Solomon

1. The minimal surface problem of three sheets, Abstract 603-136, Notices Amer. Math. Soc. 10 (1963), 472-473.

H. TALlQUiST

1. Bestimmung der Minimalflächen, welche eine gegebene ebene oder sphärische Kurve als Krïmmungskurve enthalten, Acta Soc. Sci. Fenn. 17 (1891), 473-489.

D. W. Tномpson

1. On growth and form, University Press, Cambridge, 1917.

M. TsujI

1. On a theorem of F. and M. Riesz, Proc. Imp. Acad. Tokyo 18 (1942), 172-175.

2. Potential theory in modern function theory, Maruzen, Tokyo, 1959.

J. L. Ullman and C. J. Titus

1. An integral inequality with applications to harmonic mappings, Michigan Math. J. 10 (1963), 181-192.

G. VACCARO

1. Sulle superficie d'area minima, Riv. Mat. Univ. Parma (2) 3 (1962), 139-168. G. VAN DER MENSBRUGGHE

1. Discussion et réalisation expérimentale d'une surface particulière d courbure moyenne nulle, Bull. Acad. Roy. Sci. Bruxelles (2) 21 (1866), 552-566.

2. Joseph-Antoine-Ferdinand Plateau, (Obituary), Annuaire Acad. Roy. Sci. Bruxelles 51 (1885), 389-473.

S. E. WARSCHAWSRI

1. On differentiability at the boundary in conformal mapping, Proc. Amer. Math. Soc. 12 (1961), 614-620.

K. WEIERSTRASS

1. Über die Flächen, deren mittlere Krïmmung überall gleich Null ist, Monatsb. König. Preuss. Akad. Wiss. Berlin (1866), 612-625, 855-856.

W. L. WILson

1. On discrete Dirichlet and Plateau problems, Numer. Math. 3 (1961), 359-373. W. WUNDERLICH

1. Beitrag zur Kenntnis der Minimalschraubfächen, Compositio Math. 10 (1952), 297-311.

2. Beitrag zur Kenntnis der Minimalspiralflachen, Rend. Mat. e Appl. (5) 14 (1954), 1-15.

UNIVERSITY OF MiNNESOTA 